\title{
Verification Methodology for DEVS Models
}

\author{
By
}

\author{
Hesham Saadawi
}

\author{
$\mathrm{A} \mathrm{PhD}$ thesis submitted to \\ The Faculty of Graduate and Postdoctoral Affairs \\ In partial fulfillment of \\ The degree requirements of \\ Doctor of Philosophy in Computer Science
}

Ottawa-Carleton Institute for

Computer Science

\author{
School of Computer Science \\ Carleton University \\ Ottawa, Ontario, Canada
}

Fall 2012

Copyright (C) 2012

Hesham Saadawi 
Library and Archives

Canada

Published Heritage

Branch

395 Wellington Street

Ottawa ON K1A ON4

Canada
Bibliothèque et

Archives Canada

Direction du

Patrimoine de l'édition

395 , rue Wellington

Ottawa ON K1A ON4

Canada
Your file Votre référence

ISBN: 978-0-494-94229-1

Our file Notre référence

ISBN: 978-0-494-94229-1
NOTICE:

The author has granted a nonexclusive license allowing Library and Archives Canada to reproduce, publish, archive, preserve, conserve, communicate to the public by telecommunication or on the Internet, loan, distrbute and sell theses worldwide, for commercial or noncommercial purposes, in microform, paper, electronic and/or any other formats.

The author retains copyright ownership and moral rights in this thesis. Neither the thesis nor substantial extracts from it may be printed or otherwise reproduced without the author's permission.
AVIS:

L'auteur a accordé une licence non exclusive permettant à la Bibliothèque et Archives Canada de reproduire, publier, archiver, sauvegarder, conserver, transmettre au public par télécommunication ou par l'Internet, prêter, distribuer et vendre des thèses partout dans le monde, à des fins commerciales ou autres, sur support microforme, papier, électronique et/ou autres formats.

L'auteur conserve la propriété du droit d'auteur et des droits moraux qui protege cette thèse. $\mathrm{Ni}$ la thèse ni des extraits substantiels de celle-ci ne doivent être imprimés ou autrement reproduits sans son autorisation.
In compliance with the Canadian Privacy Act some supporting forms may have been removed from this thesis.

While these forms may be included in the document page count, their removal does not represent any loss of content from the thesis.
Conformément à la loi canadienne sur la protection de la vie privée, quelques formulaires secondaires ont été enlevés de cette thèse.

Bien que ces formulaires aient inclus dans la pagination, il n'y aura aucun contenu manquant. 


\section{Abstract}

Modeling is an effective tool for studying the behaviour of a system. When modeling, the system's descriptions are usually abstracted into simpler models. These models can then be analyzed and solved (manually or automatically) by using different mathematical techniques. However, sometimes the models become too complex to analyze formally. In those cases, computer Modeling and Simulation (M\&S) can help designers to understand the behaviour of these systems better. One example of such complex systems are RealTime (RT) systems, which are usually composed of a digital computer executing software that interacts with the external physical environment with tight timing constraints.

In studying these systems, $M \& S$ has proven to be an essential tool. However, when simulating RT models, the required interactions could quickly grow beyond the ability of human observation and analysis. Instead, formal methods for verifying these systems can guarantee the correct and timely function of these systems.

Due to the benefits of formal verification of RT simulation models, this thesis introduces a methodology to enable performing formal verification of simulation models based on the Discrete Event System Specification (DEVS) formalism. The thesis introduces a road map for a complete methodology to formally verify DEVS models, thus enabling better methods for M\&S validation and verification and making $M \& S$ a better tool for RT-embedded systems development. 


\section{Acknowledgements}

I'm indebted for this work to the continuous support and encouragement of my supervisor Dr. Gabriel A. Wainer. I am grateful for his time and effort in supporting my professional and personal development. 


\section{Table of Contents}

$\begin{array}{ll}\text { Abstract } & \text { ii }\end{array}$

Table of Contents iv

List of Tables $\quad$ vii

List of Figures $\quad$ viii

Acronyms $\quad$ xi

Chapter 1: Introduction 1

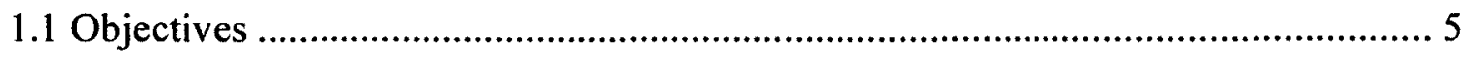

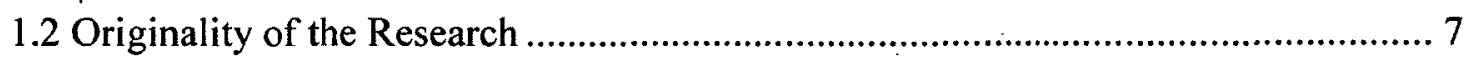

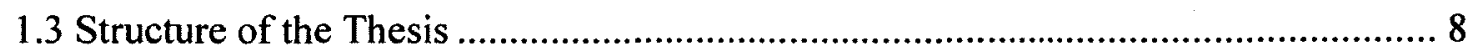

Chapter 2: Survey of State of the Art $\quad 10$

2.1 Discrete Event System Specification (DEVS) ............................................ 10

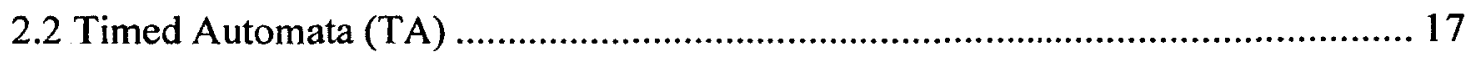

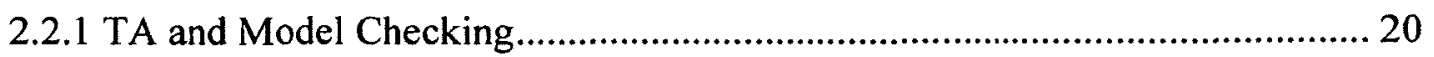

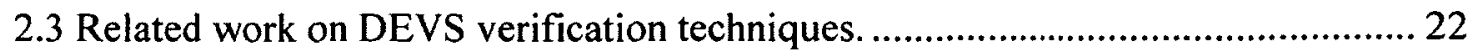

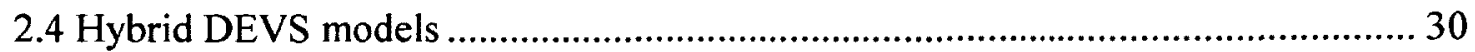

2.4.1 The Quantized State Systems (QSS) method............................................. 32

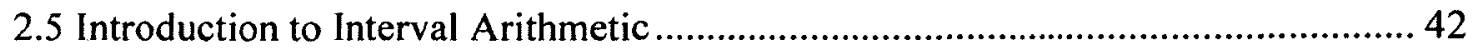




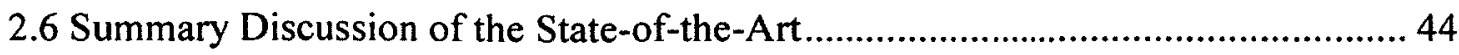

$\begin{array}{ll}\text { Chapter 3: Thesis contribution } & 46\end{array}$

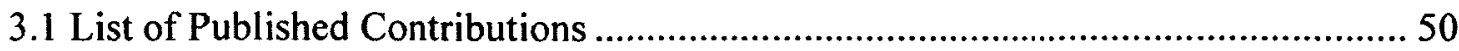

Chapter 4: The RTA-DEVS formalism

4.1 Rational Time Advance DEVS (RTA-DEVS) .............................................. 58

4.2 Estimate of Expressiveness Difference between DEVS and RTA-DEVS ............ 62

4.3 RTA-DEVS to Timed Automata Behavioural Equivalence...............................6 63

4.3.1 RTA-DEVS internal transition semantics ................................................ 73

4.3.2 RTA-DEVS external transitions semantics ...................................... 80

4.3.3 An Alternative way to show bisimulation between RTA-DEVS Internal

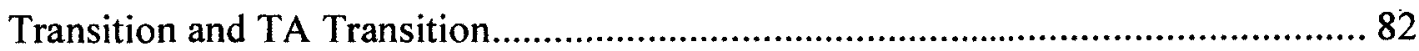

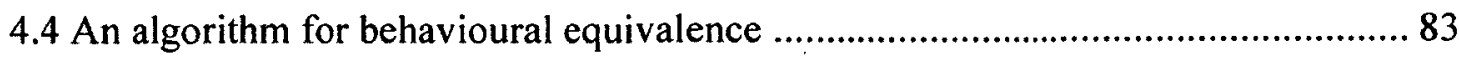

4.5 Resolving rational numbers in RTA-DEVS model ....................................... 87

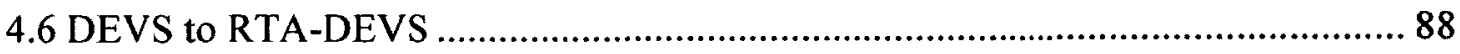

4.7 Estimation of Approximation to Verification Results ........................................ 91

4.8 Case study: an Elevator Control System....................................................... 95

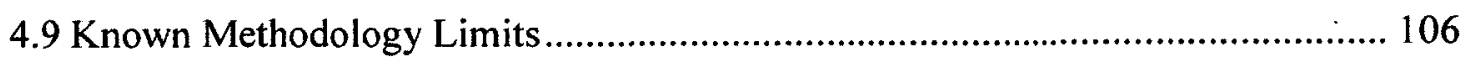

Chapter 5: Hybrid DEVS Systems Verification. $\quad 107$

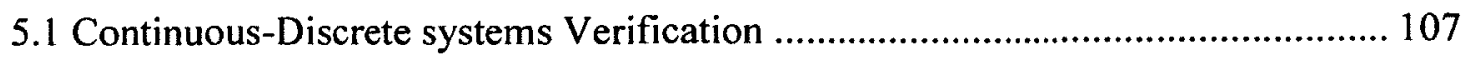

5.1.1 Transforming QSS DEVS models to TA ............................................ 112

5.1.2 Case Study of a Hybrid Elevator System ........................................... 120 


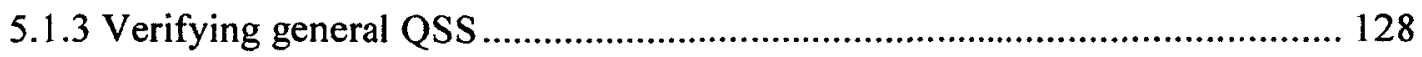

5.1.4 Advantages of using QSS/DEVS to verify Hybrid Models ....................... 147

Chapter 6: Conclusion \& Future Research $\quad 148$

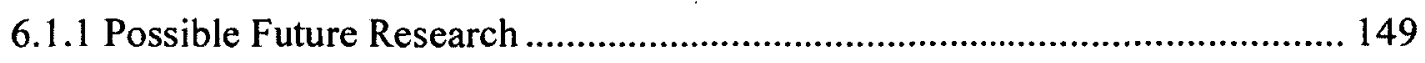

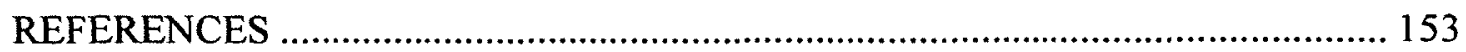




\section{List of Tables}

Table 1: Related Work of DEVS Verification and Contribution.................................. 28

Table 2: Transformation of RTA-DEVS to Behaviourally Equivalent TA................... 87

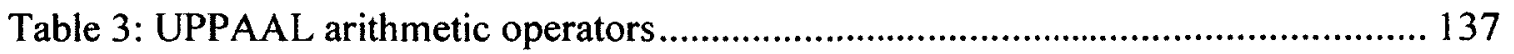

Table 4: User defined functions for the UPPAAL TA model of figure $48 \ldots \ldots \ldots \ldots \ldots \ldots . . . . . .140$

Table 5: User defined functions for the UPPAAL TA model of figure $49 \ldots \ldots \ldots \ldots \ldots \ldots . . . . .142$ 


\section{List of Figures}

Figure 1: DEVS atomic component state transition sequence (extracted, with............... 13

Figure 2: DEVS Model Hierarchy. ................................................................... 15

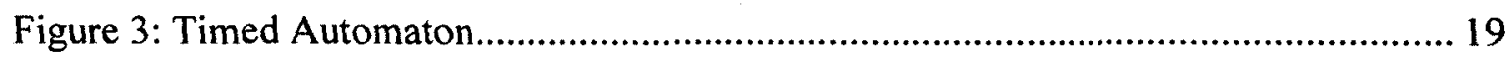

Figure 4: Quantization Function with Hysteresis (extracted, with permission, from [51]).

.

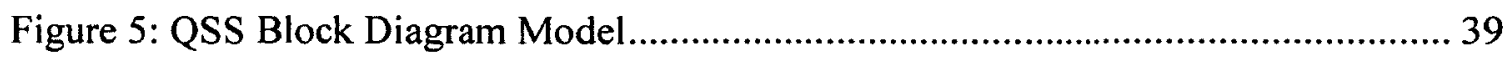

Figure 6: Continuous function of exponential Decay ........................................... 41

Figure 7: Linear approximation of Decay formula ................................................ 41

Figure 8: Quantized representation of Exponential Decay ....................................... 42

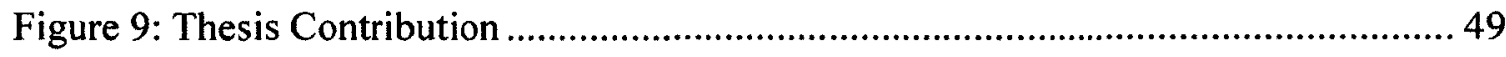

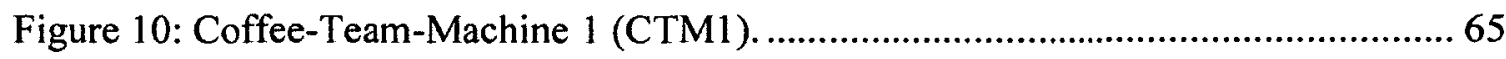

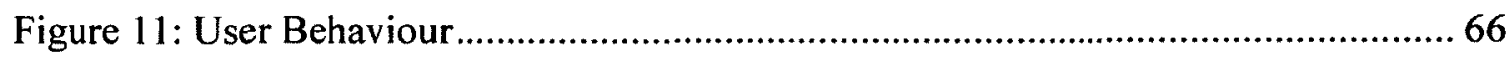

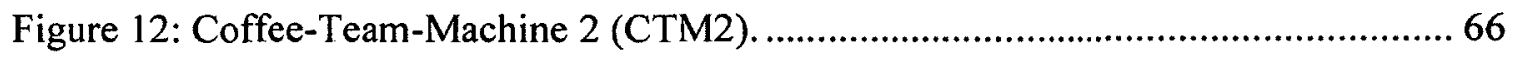

Figure 13: (a) RTA-DEVS model. (b) Timed Automata model ..............................68

Figure 14: Direct delay transition from $s_{1}$ to $s_{1}^{\prime}$ and corresponding eventual delay

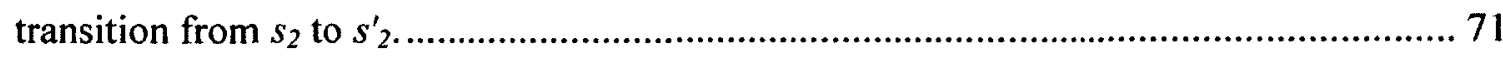

Figure 15: Direct action transition from $s_{l}$ to $s_{l}^{\prime}$, and corresponding eventual action...... 72

Figure 16: Direct delay transition from $s_{2}$ to $s_{2}^{\prime}$ and corresponding eventual delay

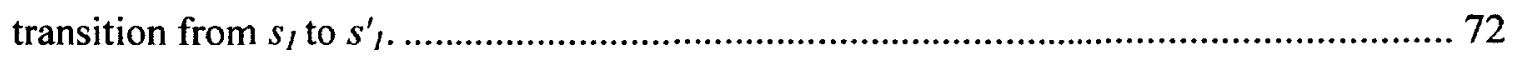

Figure 17: Direct action transition from $s_{2}$ to $s_{2}^{\prime}$, and corresponding eventual action ...... 73

viii 


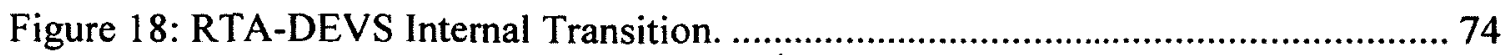

Figure 19: TA model for an Internal RTA-DEVS transition............................................ 75

Figure 20: RTA-DEVS External transitions on action a. ................................................. 80

Figure 21: TA model for RTA-DEVS external transition. .............................................. 81

Figure 22: A coupled DEVS model............................................................................ 90

Figure 23: RTA-DEVS component with External Input. .................................................. 91

Figure 24: Overlap of two intervals because of approximating irrational value. …..........94

Figure 25: Elevator RTA-DEVS Model. ..................................................................... 96

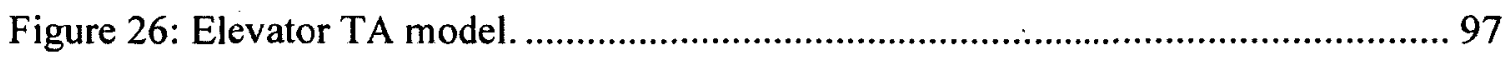

Figure 27: Elevator Controller Model as DEVS Graphs................................................ 98

Figure 28: TA Controller model in UPPAAL .................................................................. 99

Figure 29: Environment inputs (Button and Sensor). .................................................... 101

Figure 30: Elevator Verification Results in UPPAAL ................................................. 102

Figure 31: Elevator-Controller in DEVS Graphs notation with irrational value............ 104

Figure 32: TA Controller model with Non-deterministic behaviour.............................. 105

Figure 33: Exact solution for the exponential decay formula....................................... 109

Figure 34: Quantized representation of exponential decay........................................... 112

Figure 35: Example of real numbers over-approximation with integer numbers........... 116

Figure 36: TA model of exponential decay formula................................................... 119

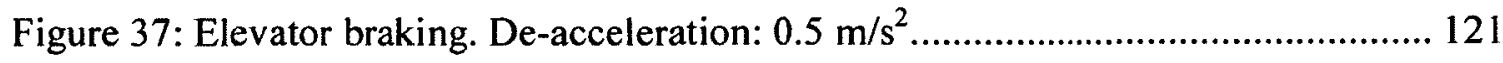

Figure 38: Elevator speed under braking. ……………............................................. 121

Figure 39: Quantized braking-elevator speed. ............................................................ 122 
Figure 40: TA model of braking elevator motion...................................................... 123

Figure 41: Modified elevator TA model- scale of 1/100 second................................. 124

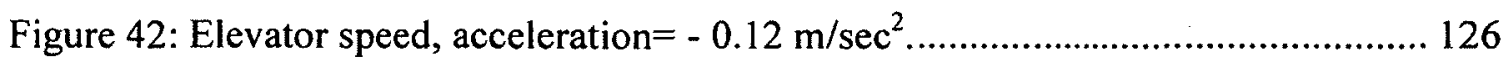

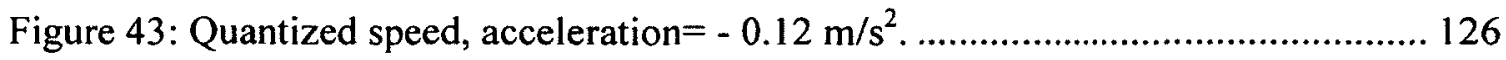

Figure 44: Exact solution for exponential decay formula............................................. 129

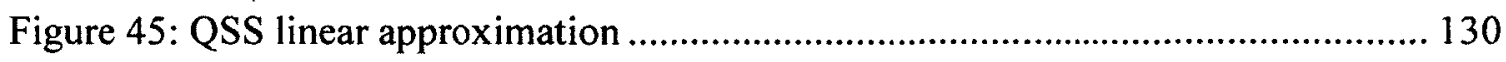

Figure 46: Over-approximation of QSS trace ............................................................. 131

Figure 47: Safety zones with QSS over-approximation. ............................................ 132

Figure 48: TA model of a QSS general Integrator ....................................................... 138

Figure 49: TA Model of a QSS Input function generation ........................................... 140

Figure 50: Definition template of $u(t)$ in UPPAAL. .................................................. 146 

Acronyms
DEVS Discrete EVent System Specification
P-DEVS Parallel DEVS
RT-DEVS Real-Time DEVS
FD-DEVS Finite and Deterministic DEVS
SP-DEVS Schedule Preserving DEVS.
M\&S Modeling and Simulation
RC Root Coordinator
TA Timed Automata
E-CD++ Embedded CD++
IDE Integrated Development Environment
GGAD Generic Graphical Advanced environment for DEVS modeling and simulation
RTA-DEVS Rational Time Advance DEVS
QSS Quantized State Systems method
STDEVS Stochastic DEVS
CTL Computation Tree Logic
PTCTL Probabilistic Timed computation Tree Logic
RT Real-Time
RTS Real-Time Systems 


\section{Chapter 1: Introduction}

Real-Time (RT) systems are advanced computer systems with hardware and software components with timing constraints. In some cases, they have "soft" timing constraints in which a deadline can be missed without serious consequences. In other cases, the system must satisfy "hard" timing constraints, and a missed deadline can result in catastrophic consequences. In these highly reactive systems, not only is correctness critical, but also the timeliness of the executing tasks. Embedded RT software systems are increasingly used in mission critical applications, where a failure of the system to deliver its function can be catastrophic. For instance, if we consider the design decisions made for an aircraft autopilot, or a controller for an automated factory, we need to obtain system responses within well-defined deadlines. Great care must be taken when developing RT systems to guarantee functional correctness along with non-functional correctness such as timing constraints.

Because of the growing complexity of RT systems and their need for high reliability, RT software development is still time-consuming, error-prone, and expensive, requiring a difficult and costly development effort with no guarantee for a bug-free software product. Many techniques have been proposed and used in practice to check correctness of RT software. Current RT engineering methodologies use modeling as a method to study and evaluate different system designs before building the real application. In this way, real 
systems would have a very high predictability and reliability. In order to apply this methodology, a designer must abstract the physical system at hand and build a model for it, then combine this with a model of the proposed controller design. Then, different techniques can be used to reason about these models and gain confidence in their correctness. Informal methods usually rely on extensive testing of the systems based on system specifications [1]. These methods have limitations because, in order to guarantee software reliability, we need to apply exhaustive testing to the software component, using all possible input combinations, which is a costly process. Many techniques have been proposed to enable a practical alternative to this exhaustive software testing [2]. However, we cannot guarantee a full coverage of all possible execution paths in a software component, thus leaving us with limited confidence in software correctness. Therefore, informal techniques can reveal errors, but they cannot prove the non-existence of errors.

The use of formal software analysis is growing as an alternative, as this technique allows full verification that software components are free of errors. In the last few decades, these techniques have matured and been used in some industrial capacity for software and hardware correctness verification [3]. Formal techniques can be used to prove the correctness of systems specifications. Nevertheless, they are usually constrained in their application, as they do not scale up well. Likewise, the designers need a high level of expertise to apply these techniques. Another drawback of formal techniques is their need to be applied to an abstract model of the real system, which 
means that what is being verified is not the final implementation. Even if the abstract designed model is proven correct, there is a risk that some errors creep in during the development process through the manual implementation of the design into executable code [1].

Formal verification techniques are of two main types, deductive or algorithmic [4]. Deductive techniques rely on representing the system and its specification with logic rules, and then trying to deduct a proof of system correctness. In this method, the user needs to find a sequence of deductions to reach the proof; hence, deductive technique needs more creativity and expertise with formal methods from the user than algorithmic one. This becomes a disadvantage with the growing size of systems, as manual intervention, required from the user, also grows. The advantage of this technique, however, is that it can deal with systems of infinite state space, which usually is the case found in hybrid systems. Algorithmic techniques rely on modelling the system in a graphical form, and coding the specifications in logical queries. Then an algorithm for reachability analysis searches the graph space for nodes that satisfy specification queries and are reachable from the initial system configuration. This method is also called model checking. For a system composed of multiple components, the model checking algorithm combines these models to build one graph representing the system overall behaviour that is called a reachability graph. By traversing this graph, the model checking algorithm can check the satisfaction of a given query against the given system model. However, for the algorithm to terminate, the reachability graph must be finite, otherwise termination would 
not be guaranteed, and the model checking problem would be undecidable. The advantage of this method is its complete automation, and the user does not need to be an expert in formal methods. New theoretical advances in model checking allow engineers to guarantee certain properties about the models of such systems using a formal approach. Model checking techniques can be automated to improve the work of the software engineer. Timed Automata (TA) theory [5], in particular, has provided many practical results in this area. However, there is still a gap between a system model that is checked as an abstract entity, and the actual system implementation code to be run on a target platform. More work is still needed to prevent errors from creeping into the final implementation as the programmer translates the requirements captured and modeled in TA into code. TA and other formal methods have showed promising results, but they are still difficult to apply and have limited power when the complexity of the system under development scales up.

A different approach to deal with these issues considers using Modeling and Simulation (M\&S) to gain confidence in the model correctness. The use of M\&S is not new, and systems engineers have often relied on these methods in order to improve the study of experimental conditions during model definition. The construction of system models and their analysis through simulation reduces both end costs and risks, while enhancing system capabilities and improving the quality of the final products. M\&S let users experiment with "virtual" systems, allowing them to explore changes and test dynamic conditions in a risk-free environment. This is a useful approach, especially 
considering that testing under actual operating conditions may be impractical and, in some cases, impossible. Nevertheless, no practical, automated approach exists to perform the transition between the modeling and the development phases, and this often results in initial models being abandoned, resulting in increased initial costs that project managers try to avoid. Simultaneously, M\&S frameworks are not as robust as their formal counterparts.

Using M\&S for RT systems enables testing real life scenarios, even for those cases in which real-life testing might be too costly or impossible to achieve [6]. If the models used for M\&S were formal, their correctness would also be verifiable, and a designer could see the system evolution and its inner workings even before starting a simulation. Another advantage of executable models is that they can be deployed to the target platform, thus giving the opportunity to use the model not only for simulations, but also as the actual implementation deployed on the target hardware. This avoids any new errors that could appear during the implementation from transformation of the verified models into an implementation, thus guaranteeing a high degree of correctness and reliability.

\subsection{Objectives}

Considering the issues in the previous section, RT system designers need a methodology to help them in the modeling and analysis of any complete design of an RT system. It is useful if the same methodology can be used to formally verify a system against its requirements, and also used to simulate individual scenarios. Finally, after complete 
verification of the correctness of the designed system, the methodology should ensure that the exact verified design could be deployed and executed on the target platform.

The objective of this thesis is to provide such a practical methodology to build and verify RT systems. This methodology uses two main formalisms to model systems specifications. The first one of them is the Discrete Event System Specifications (DEVS) formalism [7], which is based on systems theory. DEVS is the most general discrete event specification, and one can build complex models as a composite of different methods for the various components (Cellular Automata, Petri Nets, Timed Finite State Machines, Modelica, PDEs and other continuous components) that can be then translated into a DEVS representation. These DEVS models can then be simulated using DEVS abstract simulation algorithms. DEVS simplifies building complex models by its hierarchical building of coupled models out of atomic models. DEVS models are directly executable on many DEVS simulators including an RT DEVS kernel such as e-CD++ [8]. However, DEVS simulators lack formal verification capability and this was the motivation to this thesis.

The second formalism used in our methodology is Timed Automata (TA) [5]. The TA formalism, which is based on finite automata theory, was proposed to specify RT reactive systems. TA provides a solid theory and algorithms for model checking, and many existing tools implement these algorithms (for instance, UPPAAL, Kronos and others [9], [10]). TA's main concern is building an abstract formal description of the system that is verifiable by model checking, and is not focused on simulating discrete systems; thus, its 
tools lack many functions that usually exist in DEVS simulators and allow simulating different systems.

Given the strengths and weaknesses of these two formalisms, the objective of this thesis is to develop a methodology that combines.both formalisms to allow the designer to model, simulate, verify, and deploy RT systems. This is achieved by guaranteeing the correctness of the model with a methodology that verifies DEVS models with TA modelchecking techniques and tools. The verified DEVS models would then execute directly on an RT DEVS kernel, thus eliminating the risk of introducing errors in the final system implementation on the target platform. This methodology gives a model-based approach in which the user can move the original verified models to a target platform to execute them in RT without any changes. The resulting systems developed with this methodology would have a higher correctness and reliability of the actual code executing in the RT system. During this research this concept is demonstrated in actual elevator controllers running in real-time scenarios.

\subsection{Originality of the Research}

The originality of this research relies in the introduction of a new practical methodology to verify DEVS models. The methodology introduced here differs from other existing approaches in that it targets the verification of classic DEVS models and not a subset of DEVS formalism. This allows the modeller to use the classic full DEVS formalism and makes this methodology a good option to be used in an embedded systems development lifecycle methodology. 
The verification of DEVS introduced in this thesis is done on multiple stages. First, it defines a new class of DEVS, called RTA-DEVS, which is close to classic DEVS in semantics and expressive power. Then, the methodology defines a transformation to obtain a TA that is behaviourally equivalent to RTA-DEVS. The advantage of doing so is that many classic DEVS models would satisfy the semantics of RTA-DEVS models. Thus, they could be simulated with any DEVS simulator. Likewise, it could be transformed to TA to validate the desired properties formally. RTA-DEVS is close to general DEVS, in its expressiveness; nevertheless, as we will show later in the methodology, it is a verifiable subset of DEVS.

The second stage in the verification deals with the approximations and abstractions carried out for transforming DEVS models into RTA-DEVS. To assess their effect on verification accuracy, we propose a method to estimate if any errors were introduced during the transformation that may affect the verification step, or the validity of the resulting RTA-DEVS model.

A new approach is also introduced to build on the above results to enable the formal verification of hybrid systems (composed of discrete and continuous components) built with DEVS formalism. All of these research topics are original research ideas that have not been found in the literature of this domain.

\subsection{Structure of the Thesis}

This thesis is structured as follows: Chapter 2 provides the reader with a review of the state of the art of literature related to the research. In this chapter, we also introduce the 
main concepts used, including an introduction to the DEVS formalism, related work of different approaches used to verify DEVS models, an introduction to Timed Safety Automata, and to the QSS method, which will be used to model continuous systems with discrete DEVS models. We also introduce a summary of this thesis contribution in this chapter.

Chapter 3 presents the proposed RTA-DEVS formalism, and it shows how it is mapped to TA for the purpose of formal verification. An example is introduced to show the proposed methodology and algorithm. We also describe a method to transform DEVS models to RTA-DEVS with the ability to tell if this transformation would cause an effect to verification results.

Chapter 4 discusses the work to verify hybrid systems built with Quantized State Systems method QSS and DEVS components.

In chapter 5 , we present the conclusion for this research, and potential future research. 


\section{Chapter 2: Survey of State of the Art}

In this chapter, we will introduce a brief state of the art related to our thesis research. This includes a brief introduction to DEVS formalism, coverage of different techniques that have been used in literature for verification and testing of DEVS models, a brief introduction to timed automata theory and formalism, and an introduction to a topic of interest to our methodology that is modeling hybrid systems with a discrete event representation using DEVS components.

These topics would constitute an essential background to understand our methodology and to introduce our thesis in the following chapters.

\subsection{Discrete Event System Specification (DEVS).}

DEVS [7] is an accepted framework for understanding and supporting the activities of modeling and simulation. DEVS is a sound formal framework based on systems theory. It includes well-defined coupling of components, hierarchical, modular construction, support for discrete event approximation of continuous systems, support for stochastic systems, and enables component reuse. DEVS theory provides a rigorous methodology for representing models, and it does present an abstract way of thinking about the world independent of simulation mechanisms, underlying hardware and middleware. A real system modeled with DEVS is described as a composite of sub-models, each of them being atomic (behavioural) or coupled (structural). 
The basic modelling unit of DEVS is called an atomic model. A DEVS atomic component is formally defined as:

$$
A M=<X, S, Y, \delta_{\text {ext }}, \delta_{\text {int }}, \lambda, t a>
$$

Where:

$X$ : a set of external input event types

$S:$ a sequential state set

$Y:$ an output set

$\delta_{\text {exi }}: Q \times X \rightarrow S$, an external transition function

Where $Q$ is the total state set of $M=\{(s, e) \mid s \in S$ and $0 \leq e \leq t a(s)\}$

$\delta_{\text {int }}: S \rightarrow S$, an internal transition function

$\lambda: S \rightarrow Y$, an output function

ta: $S \rightarrow \mathfrak{R}^{+}, \infty$, a time advance function

Where the $\mathfrak{R}_{0, \infty}^{+}$is the non-negative real numbers with $\infty$ adjoined.

An atomic component $A M$ is a model that is affected by external input events $X$, and it generates output events $Y$. The state set $S$ represents the unique description of the states of the model. The internal transition function $\delta_{i n t}$ and the external transition function $\delta_{\text {ext }}$ compute the next state of the model. If an external event arrives at elapsed time $e$ which is less than or equal to a value specified by the time advance function $t a(s)$, a new state $s^{\prime}$ is computed by the external transition function $\delta_{\text {ext }}$. Then, a new $\operatorname{ta}\left(s^{\prime}\right)$ is computed, and the elapsed time $e$ is set to zero. Otherwise, if no external event arrives before the state lifetime elapses, a new state $s^{\prime}$ is computed by the internal transition function $\delta_{\text {int }}$. In the 
case of an internal event, the output specified by the output function $\lambda$ is produced based on the state $s$. As before, a new $t a\left(s^{\prime}\right)$ is computed, and the elapsed time $e$ is set to zero.

Figure 1 illustrates the state transition of an atomic component. This diagram describes the behaviour of an atomic model as sequence of steps indicated by the numbers on the diagram. In the first step (1) on the diagram, the atomic component is in state $s$ for a specified time $\operatorname{ta}(s)$. If the atomic component passes this time without interruption, it will execute its output function $\lambda(s)($ step 2$)$ to produce an output $y$ (step 3) at the end of this time and will change state based on its $\delta_{\text {int }}$ function (complete transition) to state $s^{\prime}$ in step 4 , and then this new state becomes the component state and we consider it as state $s$ which now is the component state (this is indicated by the dotted arrow from $s^{\wedge}$ to $s$ on the bottom of the diagram). Continuing from this new state $s$, if the component receives an input $x$ (step 5) during the state $s$ time life $\operatorname{ta}(s)$ it will change its state which is determined by its $\delta_{\text {ext }}$ function (step 6) and does not produce an output (incomplete transition). Again we consider this new state the component state $s$ (this is indicated by the dotted arrow from $s^{\wedge}$ to $s$ on the left of the diagram) and the component waits in this state for the next event to repeat the previous cycle. 


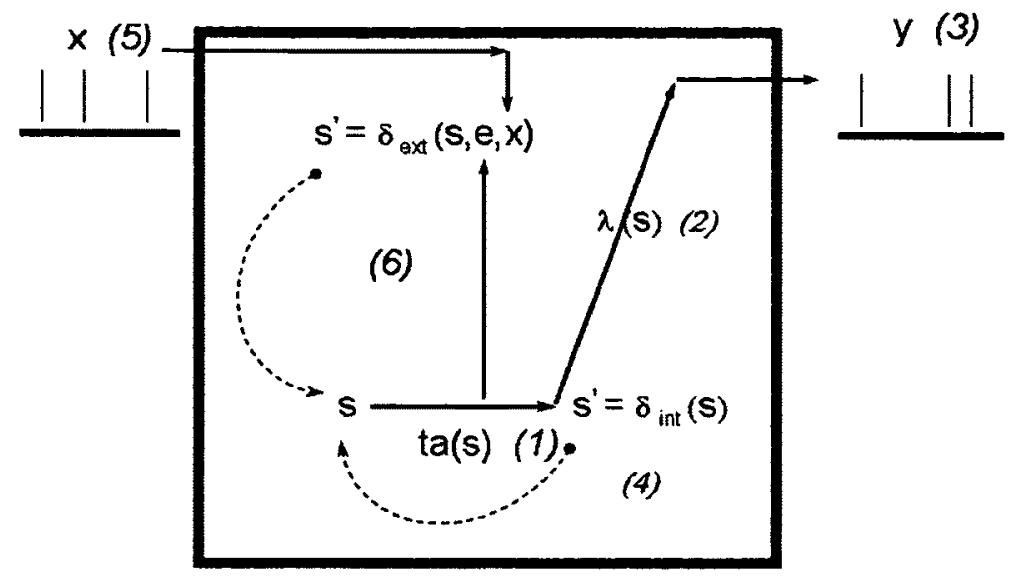

Figure 1: DEVS atomic component state transition sequence (extracted, with permission, from [7]).

A coupled model connects the basic models together in order to form a new model. This model can itself be employed as a component in a larger coupled model, thereby allowing the hierarchical construction of complex models. The coupled model is defined as:

$$
N=<X, Y, D,\left\{M_{d} \mid d \in D\right\}, E I C, E O C, I C, \text { SELECT }>
$$

Where:

$X=\left\{(p, v) \mid p \in I\right.$ Ports, $\left.v \in X_{p}\right\}$ is the set of input ports and input values for these ports; $Y=\left\{(p, v) \mid p \in\right.$ OPorts, $\left.v \in Y_{p}\right\}$ is the set of output ports and output values for these ports;

$D$ is the set of the component names.

- Each component $d$ is a DEVS model, we express each component as $d \in D$ with its DEVS atomic model defined as: 


$$
\begin{aligned}
& M_{d}=\left(X_{d}, Y_{d} S, \delta_{\text {int }}, \delta_{\text {ext }}, \lambda, t a\right) \\
& \text { Where } X_{d}=\left\{(\mathrm{p}, \mathrm{v}) \mid \mathrm{p} \in \text { IPorts }_{d}, v \in X_{P}\right\}, \\
& Y_{d}=\left\{(\mathrm{p}, \mathrm{v}) \mid \mathrm{p} \in \text { OPorts }_{d}, v \in Y_{P}\right\}
\end{aligned}
$$

The component couplings are subject to the following requirements:

External input coupling (EIC) connects external inputs of the coupled model to component inputs,

$E I C \subseteq\left\{\left(\left(N, i p_{N}\right),\left(d, i p_{d}\right)\right) \mid i p_{N} \in I P o r t s, d \in D, i p_{d} \in I P o r t s_{d}\right\}$

External output coupling (EOC) connects component outputs to external outputs, $E O C \subseteq\left\{\left(\left(d, o p_{d}\right),\left(N, o p_{N}\right)\right) \mid o p_{N} \in\right.$ OPorts, $\left.d \in D, o p_{d} \in O P o r t s_{d}\right\}$

Internal coupling (IC) connects component outputs to component inputs,

$I C \subseteq\left\{\left(\left(a, o p_{a}\right),\left(b, i p_{b}\right)\right) \mid a, b \in D, o p_{a} \in\right.$ OPorts $\left._{a}, i p_{b} \in I P o r t s_{b}\right\}$

SELECT: $2^{M} \rightarrow M$, the tie-breaking selector

A coupled DEVS model is defined with the restriction that no feedback loop is allowed, i.e. no output port of a component should be connected to one of its input ports.

A coupled model $N$ consists of components $M_{i}$ which are atomic components and/or coupled models. The influences $I_{i}$ and the $i$-to-j output translation $Z_{i, j}$ define three types of coupling specifications as follows. The external input coupling EIC connects the input events of the coupled model itself to one or more of the input events of its components. The external output coupling EOC connects the output events of the components to the output events of the coupled model itself. The internal coupling IC connects the output events of the components to the input events of other components. In these definitions, $I_{i}$ 
represents set of components that are connected to the input, while $Z_{i, j}$ represents any connections from an output of a component to the input of another component. The SELECT function is used to order the processing of the simultaneous events for sequential events. Thus, all the events with the same time in the system can be ordered with this function.

Figure 2 shows a hierarchical DEVS model. This model is composed of two atomic components (Generator, Buffer and Processor) and two coupled models: the top model contains the generator atomic component and the BUF-PROC coupled model, the BUFPROC coupled model includes two atomic components: BUF and PROC. The port connections are also visible in the figure. For example the output port "out" of atomic component PROC is connected to the "done" input port of the BUF atomic component within the same coupled model and is also connected to the output port of its parent coupled model which connects this output to the Top model output port.

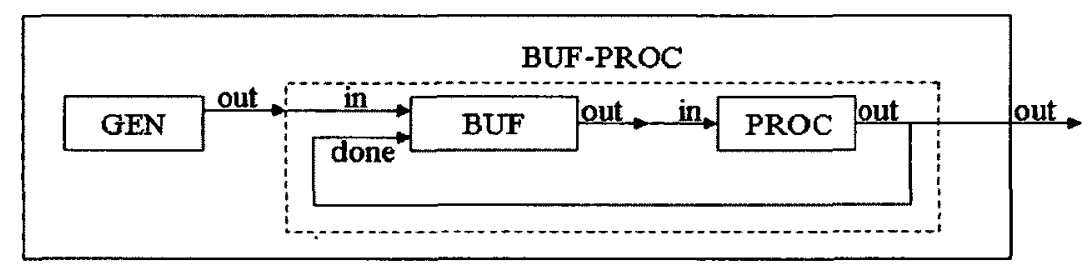

Figure 2: DEVS Model Hierarchy.

DEVS models are executed inside a DEVS simulator or executer. This simulator loads the DEVS model, reads any external event that may exist, and executes the transition functions defined in the DEVS model. Any output generated from the DEVS 
model is produced to the environment from the DEVS simulator. Some examples of DEVS simulators are CD++ [11], PowerDEVS [12], adevs [13] and DEVSJAVA [14].

DEVS simulators are also available to run on embedded platforms allowing DEVS models to run as embedded software in RT. Some initial work on executing DEVS models in real time was done in [15] to execute DEVS models on a chip. More advanced simulators were developed, for example PowerDEVS [16]. PowerDEVS has the ability to execute models on a RT operating system with synchronization of simulation time to RT clock. With its ability to model continuous systems within DEVS, the RT execution of DEVS allows simulation of physical systems in RT.

RTDEVS/CORBA [17] is a RT simulator designed to model and simulate large-scale distributed RT systems. This simulator supports the concept of model continuity that means the same model of a system can be used through all design phases. This simulator provides RT modeling \& simulation environment so that the system under study can be simulated with its environment model in real-time. Zeigler in [7] describes the internal architecture of such RT simulator and some differences of RT simulator from the classic DEVS simulator to increase performance and enable interaction with underlying operating system to support concurrent execution threads.

E-CD++ $[8]$ is an embedded DEVS simulator that can execute DEVS models in RT and interacts with external sensor and actuators as shown in a case study in [18]. Using DEVS models as executable models on an embedded platform closes the gap between modeling and implementation, thus avoiding errors that may be introduced by the manual 
translation of models into executable code. This approach can be greatly enhanced with the ability to validate DEVS models not only through simulation, but also with the ability to formally verify the model against the initial requirements. In this case, DEVS models would be proven free of defects, and its direct execution on embedded platform eliminates any possible creep of defects into the executed code. The goal of this thesis therefore, is to present a robust methodology to formally verify DEVS models using different formal techniques and methods, and giving the system modeller a robust method to estimate the effect of any approximations necessary to achieve that goal.

\subsection{Timed Automata (TA)}

A Timed Automaton can be defined as in [10]:

$$
A=\left(N, \mathbf{l}_{\mathbf{o}}, \mathbf{E}, \mathbf{I}\right)
$$

$-N$ is a finite set of locations (or nodes),

$-l_{o} \in N$ is the initial location,

$-E \subseteq N \times \beta(C) \times \sum \times 2^{C} \times N$ is the set of edges and

$-I: N \rightarrow \beta(C)$ assigns invariants to locations

Here, $C$ is a set of clock variables (with $x, y, \ldots$ representing clock variables from the set $C)$. We use $a, b$, etc. to represent the actions from a set of the finite alphabet $\Sigma$. Let us assume a finite set of real-valued variables $C$ ranged over by clocks $x, y, \ldots$ and a finite alphabet $\Sigma$ (with actions $a, b, \ldots$ ). Let us call a clock constraint to a conjunctive formula of atomic constraints of the form $x \sim n$ or $x-y \sim n$ where $x, y$ are clock variables, $\sim$ is one of $\{\leq$, $<,=,>, \geq\}$ and $n$ is a natural number. The clock constraints can be used on transitions, 
where they are called guards; or in a location (state), where they are called invariants. Invariants are constraints on the form $x \leq n$, or $x<n$ to restrict time spent in a TA location. $\beta(C)$ denotes set of clock constraints. We use the notation $u \dashv I(l)$ to mean that clock valuation $u$ satisfies the invariant defined in location $l$. We also define a TA state as a pair of (location, clock valuation) such as $(L, u)$.

TA are Timed Transition Specifications in which the states are pairs $\langle L, u\rangle$, where $L$ is a location, and $u$ is a clock valuation. We write $l \stackrel{g, a, r}{\longrightarrow} l^{\prime}$ when $(l, g, a, r, l) \in E$ with $g$ a clock constraint, $a$ an action, and $r$ a set of clocks to be reset to zero.

TA uses two types of transitions:

- Delay Transitions: $\langle L, u\rangle \stackrel{d}{\longrightarrow}\langle L, u+d\rangle$, where the time passage $d$ causes a transition from a start location $L$ to an end location $L$ if $u \dashv I(L)$ and $(u+d) \dashv I(L)$.

- Action transitions: $\left\langle L, u>\stackrel{a}{\longrightarrow}<L^{\prime}, u^{\prime}\right\rangle$, where an action $a$ causes a transition from a start location $L$, to an end location $L^{\prime}$, and $u^{\prime} \nmid I(L)$. 


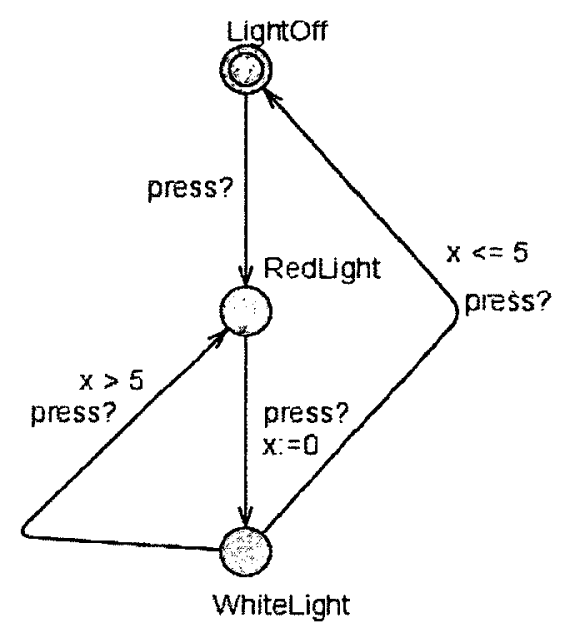

Figure 3: Timed Automaton

An example of a timed automaton is shown in figure 3. This model represents a light that can be either off, operating in red mode, or in white mode. The light changes modes depending on the user pressing the light switch button. From the red mode if the switch is pressed twice within 5 seconds, the light goes to white then to off, otherwise it goes to white then back to red. In order to do that, the TA uses three states. LightOff is the initial state, which represents that the light is turned off. The transition out of WhiteLight to LightOff has the a guard $x \leq 5$ which enables the transition only while clock $x$ value stays less than 5 time units, whenever a synchronization signal arrives on channel press. States could also have clock constraints and in this case, they are called invariants. In this case, time is allowed to pass in a state while the clock values satisfy the invariant. Once the invariant is not satisfied, the automaton would leave that state and enable a transition to another state, in which current clock values would satisfy that state invariant. TA is suitable for modeling discrete systems with continuous time. These systems could be 
composed of a single TA model, or multiple models that interact together. The latter case is called a network of TA.

\subsubsection{TA and Model Checking}

One of the most successful techniques for systems formal verification is model checking [19]. In this method, the system to be verified is modeled by a suitable formalism such as I/O automata or TA, which can be represented in a graphical notation. Each state of the model assumes some valid system property(ies) at this state.

System requirements are specified as a temporal logic formula called query. The model checking technique then uses an algorithmic approach to traverse the model graph, and it checks the satisfaction of the query against the properties defined at each state. For a system composed of multiple components, the model checking algorithm combines these models to build one graph representing the system overall behaviour that is called a reachability graph. By traversing this graph, the model checking algorithm can check the satisfaction of a given query against the given system model. However, for the algorithm to terminate, the reachability graph must be finite, otherwise termination would not be guaranteed, and the model checking problem would be undecidable. With timed formalisms such as TA, the time elapse is modeled by a continuous clock variable. This variable value increases when TA is waiting in a state. Even for a bounded Real interval, the number of Real values would be infinite as shown by the famous Cantor's diagonal method. However, TA model checking for finite state machines [20][21][22] was enabled by extending symbolic model checking techniques [23][24] to build a finite reachability 
graph with continuous time. Nevertheless, the state explosion problem still limits the size of actual problems that can be solved. The state explosion problem is a term describing the exponential growth of the reachability graph size with the growing number of components and states of each component in a network of TA. This renders the techniques of model checking practically limited to small and medium industrial problems unless combined with other techniques to overcome this exponential growth of the reachability graph.

Recent techniques to reduce this problem have been proposed and these results were implemented in a number of tools for TA model checking with a success to check models of increasing sizes. One of these tools is UPPAAL [9][10] that have extended TA with integer variables, urgent channels and user-defined functions. These extensions increase the conciseness of the model, but not the expressiveness power as shown in [25]. UPPAAL uses a subset of TCTL (Timed Computation Tree Logic) [24] to specify queries for properties in the TA model.

Timed safety Automata is the version of TA used in the UPPAAL model checker [10]. This thesis used this class of TA as it suffices for the verification purpose to represent DEVS models and it is verifiable within UPPAAL tool.

In the methodology introduced in this thesis, if UPPAAL (or any other model checker) faces a problem of state explosion, and no answers can be obtained in finite time, the user can use model checking on a rough abstract of the system. From this step, some requirements may not be satisfied in the rough abstract model. These would generate a 
trace that can be used as a seed input to a simulated mode to test the models using DEVS simulation. Subcomponents can be verified using TA model checkers, improving the overall quality of the system.

\subsection{Related work on DEVS verification techniques.}

There have been several proposals to verify DEVS models, ranging from formal modelchecking of restricted classes of DEVS, the generation of traces from DEVS models for testing, the specification of high-level system requirements in TA (and verifying DEVS model against those requirements), or introducing clock constructs to DEVS to conform with TA. In this section, we give an overview of these techniques and compare different approaches. From this comparison, we show how the methodology introduced in chapter 3 closes an existing gap in current techniques.

In [26], Wainer et al, presented the verification of DEVS models, checking the consistency of model structure, the correct coupling of components, and the correct definition of the transition functions. These checks were done statically before executing the models and introduced a component to monitor execution for some verification. However, this approach has a limited ability, as it needs to simulate all possible executions of a model to verify all interactions with the model.

In [27], Hernandez and Giambiasi showed that verification of general DEVS models through reachability analysis is undecidable. They based their deduction on building a DEVS model that simulates a Turing machine. Since the halting problem for Turing machines is undecidable (i.e. with only static analysis, we cannot know if a Turing 
machine would be in a halting state after execution), Hernandez and Giambiasi concluded that this is also true for DEVS models. Therefore, if we start from an initial state in the DEVS model, we cannot know if we would reach a particular state, and hence reachability analysis for general DEVS is impossible. However, reachability analysis would be possible only for restricted classes of DEVS with finite state space, as the undecidability result was obtained by introducing state variables into DEVS formalism with infinite number of values.

Because of the above undecidability result, many approaches have created restricted DEVS subclasses that are verifiable, and in some cases extend original DEVS definition with specific properties for RT systems.

One of these approaches is the Real-Time DEVS formalism (RT-DEVS) [28] that extends classic DEVS definition by introducing a time advance function that maps each state to a time-range with maximum and minimum time values. In [29], RT-DEVS was used to model a RT system of train-gate-controller. Song and Kim introduced an algorithm to build a timed reachability tree to check model safety analysis. This work however did not focus on restricting infinite state-space of DEVS for reachability analysis decidability, and it assumed practical RT-DEVS models simulated with modern computers to be an approximation of general DEVS that enables decidable reachability analysis.

In another approach, Hwang defined a subclass of DEVS to enable verification analysis of its models. This was called Schedule-Preserving DEVS (SP-DEVS) 
[30][31][32][33]. SP-DEVS puts the following restrictions on DEVS to obtain finite reachability graph and thus a decidable reachability analysis:

- The sets of states, input, and output events are finite.

- The lifespan of a state can only be a rational number or an infinite time value.

- Preserving the internal transition function schedule after taking any external transition, i.e. if a state transition is caused by an input event, the lifespan and elapsed time are preserved after moving to the new state [33].

These restrictions limited the expressiveness of SP-DEVS to be less than that of classic DEVS; in particular, the last restriction above. Moreover, this restriction caused a problem known as OPNA (Once an SP-DEVS model becomes Passive, it Never returns to become Active), as shown in [30]. This problem results from the restriction of preserving the schedule of the internal transition. Hence, if that schedule was infinity, any subsequent external event will not be able to change it; thus, any passive state can never be interrupted (by the means of assigning a finite time advance value to it).

To solve these issues with SP-DEVS, another sub-class of DEVS was introduced, called Finite-Deterministic DEVS (FD-DEVS) [34]. In this work, Hwang mapped the time advance function states only to rational numbers, and prohibited the external transition function to use the elapsed time to compute its result. It also removed the previous restriction of SP-DEVS of preserving the internal schedule, thus avoiding the OPNA problem. With the other restrictions, reachability analysis of FD-DEVS became decidable. Hwang in [34] also introduced an algorithm for verification through 
reachability analysis similar to the algorithms and techniques used for TA. However, the restrictions introduced in FD-DEVS for the definition of its external function restricted the expressiveness of FD-DEVS to be much less than classic DEVS. The reachability analysis algorithm introduced for FD-DEVS uses similar techniques as TA, while Hwang and Zeigler in [34] have not claimed an advantage over TA reachability algorithms and tools in time and space complexity. Thus, this approach lacks the support of robust TA model checking tools like UPPAAL or KRONOS, without gaining a tangible advantage.

Another approach for verifying another class of DEVS which is RT-DEVS, is done using TA and UPPAAL. Using this approach, Furfaro and Nigro[35][36] and Cicirelli and Furfaro [37] introduced a transformation from RT-DEVS to UPPAAL. This transformation allows Weak synchronization between components of TA model as RTDEVS semantics uses Weak synchronization. Once the transformation is done, one obtains a TA model that can be verified with available tools for TA model checking. However, the transformation given did not formally show an equivalence of timed behaviour between RT-DEVS and TA models, and did not introduce a mechanism to approximate irrational values that may be defined in RT-DEVS models, or how to evaluate the impact of such approximation on verification results.

The work presented in [38] by Han and Huang used a different approach to verification of classic parallel DEVS models, based on a method to map DEVS models to TA. However, this was different from the previous approaches, as in this approach, the conversion method mapped both DEVS model and also a representation of the DEVS 
simulator to TA. The approach suggests trace equivalence as the basis for parallel DEVS and TA model equivalence. This approach considers not only the DEVS model, but also the execution engine semantics and structure to be part of the transformation to TA. As Zeigler et al. shown in [7], in a DEVS simulator, every atomic component has a coordinator component responsible for routing messages from and into the atomic component. Hence, in the verification approach of [38], each atomic model translated to a TA would also need a model of its coordinator translated to TA. Thus, a coupled model composed of $n$ atomic DEVS components translated to TA by this approach would also need at least $n+1$ local coordinators interacting with the global coordinator of the simulator. As each introduced coordinator contains few states and transitions to model its behaviour, the introduction of these $n+l$ coordinators increases the total number of states in the resultant TA model exponentially, and this adds to the state-space explosion problem. This would limit the practical size of any problem that can be verified by this approach. Another drawback is the notion of trace equivalence between Timed Transition Systems (TTS) as shown by Aceto et al. in [39] . This equivalence is less suitable for reactive systems as it may not reveal subtle errors in the models that would not be observed externally. This was shown in [39] by Aceto et al., as well it was shown that only bi-simulation equivalence can reveal such errors. Also Timed Computational Tree Logic TCTL, which is used to model queries in timed model checking tools such as UPPAAL and Kronos, is not preserved by trace equivalence. This means TCTL queries that are satisfied on TA model may not be satisfied on the original DEVS model. 
Dacharry and Giambiasi [40] introduced a similar approach based on TA to verify subclasses of DEVS formalism. They introduced a new class of DEVS called Time Constrained DEVS (TC-DEVS), which expanded the DEVS atomic model definition with multiple clocks incremented independently. Classic DEVS atomic models can be seen as having only one clock that keeps track of elapsed time in a state, and is reset on each transition. TC-DEVS also added clock constraints similar to TA (functioning as guards on external and internal transitions). However, it expands on TA guards by allowing clock constraints as state invariants to contain clock differences. TC-DEVS is then transformed to an UPPAAL TA model. However, there is no restriction on the constant values defined in TC-DEVS to be rational numbers. Hence, the resulting TA model from transformation of general TC-DEVS formalism may have undecidable reachability.

Other than proposing verifiable subclasses of DEVS, some other approaches used TA to model high-level system requirements while using the DEVS formalism to model lower-level system design. Then these approaches would build a refinement relation between a DEVS model and a TA model. This approach was followed by Giambiasi et al. in [41]. System requirements are then verified through the simulation of the DEVS model. This approach differs from others in that it does not use formal methods to verify DEVS or TA models, but relies on exhaustive testing through simulation of scenarios. The problem with this is that it is very difficult to cover all scenarios for a system, and it is a resource-intensive exercise to cover even a portion of these scenarios. 
Other approaches use semi-formal DEVS verification techniques. Hong and Kim [42] and Labiche and Wainer [43] proposed to analyze the DEVS model formally, and then to generate test scenarios that can be used to verify the DEVS models. These testing scenarios or sequences are generated from model specifications and then applied against the model implementation to verify the conformance of implementation to specifications. A summary of these approaches along with this thesis approach is shown in table 1 .

Table 1: Related Work of DEVS Verification and Contribution

\begin{tabular}{|c|c|c|c|c|c|c|c|}
\hline \multirow{2}{*}{$\begin{array}{l}\text { Verification } \\
\text { Method }\end{array}$} & \multirow{2}{*}{$\begin{array}{l}\text { Modelling } \\
\text { Formalism }\end{array}$} & \multicolumn{3}{|c|}{ Ability to Verify } & \multirow{2}{*}{$\begin{array}{l}\text { Coverage of } \\
\text { classic DEVS } \\
\text { Verification }\end{array}$} & \multirow{2}{*}{$\begin{array}{l}\text { Coverage of } \\
\text { Hybrid } \\
\text { DEVS } \\
\text { Models } \\
\text { Verification }\end{array}$} & \multirow[t]{2}{*}{ Disadvantages } \\
\hline & & Safety & $\begin{array}{l}\text { Bounded } \\
\text { Liveness }\end{array}$ & $\begin{array}{l}\text { Deadlock } \\
\text { Freedom }\end{array}$ & & & \\
\hline $\begin{array}{l}\text { Static checks } \\
\text { and runtime } \\
\text { monitoring } \\
{[17]}\end{array}$ & DEVS & $\begin{array}{l}\text { NO (No } \\
\text { exhaustive } \\
\text { checking) }\end{array}$ & $\begin{array}{l}\text { NO (No } \\
\text { exhaustive } \\
\text { checking) }\end{array}$ & No & No & No & $\begin{array}{l}\text { Runtime } \\
\text { monitoring } \\
\text { cannot } \\
\text { exhaustively } \\
\text { check all } \\
\text { scenarios. }\end{array}$ \\
\hline $\begin{array}{l}\text { Reachability } \\
\text { tree } \\
\text { construction }\end{array}$ & RT-DEVS & Yes & Yes & Yes & $\begin{array}{l}\text { No treatment } \\
\text { of irrational } \\
\text { values. }\end{array}$ & No & No Tool support \\
\hline $\begin{array}{l}\text { Reachability } \\
\text { tree } \\
\text { construction }\end{array}$ & SP-DEVS & Yes & Yes & yes & No & No & No Tool Support \\
\hline $\begin{array}{l}\text { Reachability } \\
\text { tree } \\
\text { construction }\end{array}$ & $\begin{array}{l}\text { Finite- } \\
\text { Deterministic } \\
\text { DEVS (FD- } \\
\text { DEVS) }\end{array}$ & Yes & $\overline{\text { Yes }}$ & yes & No & No & $\begin{array}{l}\text { lacks the support } \\
\text { of robust TA } \\
\text { model checking } \\
\text { tools like }\end{array}$ \\
\hline
\end{tabular}




\begin{tabular}{|c|c|c|c|c|c|c|c|}
\hline & & & & & & & $\begin{array}{l}\text { UPPAAL or } \\
\text { KRONOS }\end{array}$ \\
\hline UPPAAL TA & RT-DEVS & Yes & Yes & yes & $\begin{array}{l}\text { No treatment } \\
\text { of irrational } \\
\text { values. }\end{array}$ & No & $\begin{array}{l}\text { No Proof of } \\
\text { behavioral } \\
\text { equivalence. }\end{array}$ \\
\hline TA & $\begin{array}{l}\text { classic parallel } \\
\text { DEVS [46] }\end{array}$ & Yes & Yes & yes & $\begin{array}{l}\text { No treatment } \\
\text { of irrational } \\
\text { values. }\end{array}$ & No & $\begin{array}{l}\text { Used trace } \\
\text { equivalence } \\
\text { notion which is } \\
\text { weaker than } \\
\text { bisimulation } \\
\text { equivalence. } \\
\text { Modeled } \\
\text { execution engine } \\
\text { along with the } \\
\text { system model } \\
\text { which increases } \\
\text { state-space } \\
\text { growth and } \\
\text { verification } \\
\text { complexity. }\end{array}$ \\
\hline UPPAAL TA & $\begin{array}{l}\text { Time } \\
\text { Constrained } \\
\text { DEVS (TC- } \\
\text { DEVS)[49] }\end{array}$ & Yes & Yes & yes & No & No & $\begin{array}{l}\text { No } \\
\text { transformation } \\
\text { from DEVS to } \\
\text { TC-DEVS. }\end{array}$ \\
\hline $\begin{array}{l}\text { Test cases } \\
\text { generation }\end{array}$ & DEVS[S0] & $\begin{array}{l}\text { Yes } \\
\text { (partially), } \\
\text { as it is very } \\
\text { costly }\end{array}$ & No & No & No & No & $\begin{array}{l}\text { Cannot work on } \\
\text { abstracted DEVS } \\
\text { model to reduce } \\
\text { the problem size. }\end{array}$ \\
\hline UPPAAL TA & $\begin{array}{l}\text { RTA-DEVS } \\
\text { (This thesis) }\end{array}$ & Yes & Yes & Yes & Yes & Yes & \\
\hline
\end{tabular}


The techniques we showed so far concern the verification of hard real-time systems. In these systems, we cannot tolerate any response missing its deadline. However many real-time systems can tolerate some occasional missing of deadlines, and in this case, the system can still perform its function with some degraded performance. These system are called soft real-time. In these systems, a verification question would be "with probability 0.99 or better, the system would respond within 10 time units". Answering this question requires modeling the system in a formalism that allows probabilistic behaviour such as stochastic DEVS [44] and Probabilistic Timed Automata [45].

\subsection{Hybrid DEVS models}

Hybrid models are particularly important in modeling digital control systems where the controlled environment obeys the laws of physics, while the controller can be a digital discrete system or a combination of both digital and analog. The study of such systems requires the verification of the resulting hybrid system.

A major problem in verification of hybrid systems is the lack of a unified theory to model and solve both continuous and discrete components together [46]. As a result, modeling and simulation is still one of the most useful methods to verify this kind of systems [47] [48] [49]. Hybrid systems simulation was enabled within the DEVS formalism by using a new method, called Quantizes State System (QSS) that will be covered in section 2.4.1, which allows modeling continuous components [50][51][52]. However, simulation does not guarantee the absence of defects from the system under 
study. Simulation verifies the system for particular scenarios chosen by the system tester. As many hybrid systems are embedded in nature, and their failure could cause catastrophic results, it is of most importance to verify these systems for their adherence to requirements and their absence from defects. Formal methods can be used to provide such a guarantee. In doing so, a hybrid system needs to be modeled and verified within a formal framework.

In order to use this algorithmic method (model checking through reachability analysis) to verify hybrid systems, the focus would be to find a suitable finite abstraction of the hybrid system that could be verified, and hence the reachability algorithm could be guaranteed to terminate. Different types of labelled transition systems were proposed to model hybrid systems abstractions including Petri Nets [53], hybrid automata [54] and TA [4].

Some research has used hybrid TA for modeling hybrid systems and verifying them. This type of automata describes the system with a Timed Labelled Transition System (TLTS) and linear differential equations [54]. However, as Henzinger et al. shows in [55], Hybrid TA verification through reachability analysis is not decidable in general. For this reason, recent research has concentrated on modeling the hybrid system in some form with a decidable verification such as TA. In doing so, a technique must be used to model the continuous component in a discrete finite form. As continuous system variables are real values and dense in time (i.e. time scale is continuous and we have infinitely many time points in any bounded interval), their state space could be infinite. An 
approximation to a finite representation is needed to enable the decidability and termination of reachability analysis. Many techniques have been proposed to approximate the continuous-time systems into a discrete representation of TA [56] [57][58][59].

Although DEVS is a discrete-event system specification, some work has been done to represent continuous systems in a discrete format that can be modeled and simulated with DEVS. One of these methods is Quantized State Systems (QSS) method [51]. Using QSS enables modeling and simulation of hybrid systems with DEVS formalism.

\subsubsection{The Quantized State Systems (QSS) method}

This section is devoted to give a general introduction to the QSS method, as introduced in [50] and [51]. The QSS is an approximation method to model and simulate continuous systems, which are usually modeled with Ordinary Differential Equations (ODE) and Algebraic Equations. This combination of equations describing the system behaviour is generally called Differential Algebraic Equations (DAE). Obtaining a detailed description of the system behaviour entails solving these equations simultaneously. In doing so, many different techniques of numerical integration have been proposed to solve ODEs; namely Euler, Runge-Kutta, etc [60]. These methods approximate the solution of ODEs, and they limit the error to an acceptable range based on the choice of its discrete integration step. All these methods rely on discrete-time integration of ODEs. In this way, time is allowed to progress in small steps, and at each step, an approximation is computed for the ODEs solution. When a system modeled by DAE has a discontinuity (i.e. a sudden jump in its variables values with regard to time), the numerical integration method may 
produce unacceptable errors [52]. Unfortunately, this kind of discontinuity are normal properties of hybrid systems, which can be seen as operating in different modes, each described with a specific ODEs. An example of such a system would be a heating system with an on-off thermostat switch; in this system, the ODEs describing the system in the heating state (i.e., when the thermostat is in on position) are different from those describing cooling state (i.e., the thermostat is in the off position).

The Quantized State Systems QSS [50][51][60] is a different method for approximation, a quantization-based method that models hybrid systems as discrete-event systems and not as discrete-time. In the quantization-based method, instead of fixing a time step and calculating the value of the state variable at the next time step, we calculate when the state variable reaches a certain value. This value is called a quantization level, and the difference between two quantization levels is called the quantum. Obviously with a quantization-based method, the time it takes for a state variable to reach a quantization level depends on the state variable slope. The greater the slope, the less time it takes to reach the next level. This produces a variable time-step integration, which becomes an advantage when the system has a vector of state variables, in which each variable has a different change rate (slope). In this case, a variable with large slope would generate more events per unit time, than a variable with a flat slope. As DEVS is an event-based formalism, meaning it would only react to generated or received events, thus a slowchanging variable would need less computations, than a rapidly-changing variable. This event-based property of DEVS also solves the above problem around discontinuities 
found while solving hybrid systems. In fixed time-step integration methods, the step boundary has to exactly match the point in time in which the discontinuity (or a system jump) happens. Otherwise, the integration would suffer major error if it assumes a continuous trajectory around the discontinuity and handles this in single time step. This problem however, disappears with discrete-event system simulation such as DEVS because whenever an event triggers a discontinuous transition in the system state, DEVS simulator would react to this event and calculate the system state resulting from that event as discussed in [51]. Thus, the integration slope (which is a part of the system state) before the event would be different from that after the discontinuity event. This would guarantee the correct calculation of the system variables before and after the system jump. Consider a continuous system modeled by a time-invariant Ordinary Differential Equation (ODE), and its State Equation System (SES) representation:

$$
\left.x^{\cdot}(t)=f[x(t), u(t))\right]
$$

Here $x(t) \in \mathscr{R}^{n}$ represents the system state vector such as $\left(x_{l}(t), x_{2}(t), x_{3}(t), \ldots x_{n}(t)\right)$ and $u(t) \in \mathscr{R}^{m}$ represents an input vector, which is a known piecewise constant function. With the QSS method, we simulate an approximate system, which is called the Quantized State System:

$$
\left.x^{\cdot}(t)=f[q(t), u(t))\right]
$$

Where $q(t)$ is a vector of quantized variables $\left(q_{l}(t), q_{2}(t), \ldots, q_{n}(t)\right)$ that are obtained with the quantization function $q$ from the state variables $x(t)$. 
Each component of $q(t)$ is related to the corresponding component of $x(t)$ by a . hysteretic quantization function, which is defined here as given in [51]:

“Definition 1. Let $Q=\left\{Q_{0}, Q_{1}, \ldots, Q_{r}\right\}$ be a set of real numbers where $Q_{k-1}<Q_{k}$ with 1 $\leq k \leq r$. Let $\Omega$ be the set of piecewise continuous real valued trajectories and let $x_{i} \in \Omega$ be a continuous trajectory. Let $b: \Omega \rightarrow \Omega$ be a mapping and let $q_{i}=b\left(x_{i}\right)$ where the trajectory $q_{i}$ satisfies:

$q_{i}(t)= \begin{cases}\mathrm{Q}_{\mathrm{m}} & \text { if } \mathrm{t}=\mathrm{t}_{0} \\ \mathrm{Q}_{\mathrm{k}+1} & \text { if } \mathrm{x}_{\mathrm{i}}(\mathrm{t})=\mathrm{Q}_{\mathrm{k}+1} \wedge \mathrm{q}_{\mathrm{i}}\left(t^{-}\right)=\mathrm{Q}_{\mathrm{k}} \wedge k<r \\ \mathrm{Q}_{\mathrm{k}-1} & \text { if } \mathrm{x}_{\mathrm{i}}(\mathrm{t})=\mathrm{Q}_{\mathrm{k}}-\varepsilon \wedge \mathrm{q}_{\mathrm{i}}\left(t^{-}\right)=\mathrm{Q}_{\mathrm{k}} \wedge k>0 \\ \mathrm{q}_{\mathrm{i}}\left(t^{-}\right) \text {otherwise }\end{cases}$

Where:

- $Q_{m}$ : is the initial quantization value at start of time $t_{0}$.

- $Q_{k+1}$ : is the next quantization level at time $t$ if the function slope is positive and last quantization level the function has crossed was $Q_{k}$.

- $\mathrm{Q}_{\mathrm{k}-1}$ : is the next quantization level at time $\mathrm{t}$ if the function slope is negative, the last quantization level the function crossed was $Q_{k}$, and the function value $x_{i}(t)$ is less than quantization level $\mathrm{Q}_{\mathrm{k}}$ by the hysteresis value $\varepsilon$.

- $\quad t$ is a point in time such that $t^{-}<\mathrm{t}$

The index $m$ is described by:

$$
m=\left\{\begin{array}{l}
0 \text { if } x_{i}\left(t_{0}\right)<Q_{0} \\
r \text { if } x_{i}\left(t_{0}\right) \geq Q_{r} \\
j \text { if } Q_{j} \leq x_{i}\left(t_{0}\right)<Q_{j+1}
\end{array}\right.
$$


Then, the map $b$ is a hysteretic quantization function.

The discrete values $Q_{k}$ are called quantization levels and the distance between two successive quantization levels $\left(Q_{k+1}-Q_{k}\right)$ is defined as the quantum, which is usually constant. The width of the hysteresis window is $\varepsilon$. The values $Q_{0}$ and $Q_{r}$ are the lower and upper saturation bounds. Figure 4 shows a typical quantization function with uniform quantization intervals." [51]

As shown in figure 4 , a hysteresis function approximates a continuous linear function $x_{i}(t)$ by outputting a number of discrete levels. The crossing of the continuous function to a quantization level generates an output. Notice however that the output value chosen of $x_{i}(t)$ differs when the continuous function $x_{i}(t)$ increases from that when the function decreases. This difference or delay is the hysteresis window $\varepsilon$. Hence an output is dependent not only on the value of the function $x_{i}(t)$, but also on the history preceding this value. The use of hysteresis function is typically to prevent rapid switching of output . if value of $x_{i}(t)$ fluctuates around some quantization level $Q_{i}$.

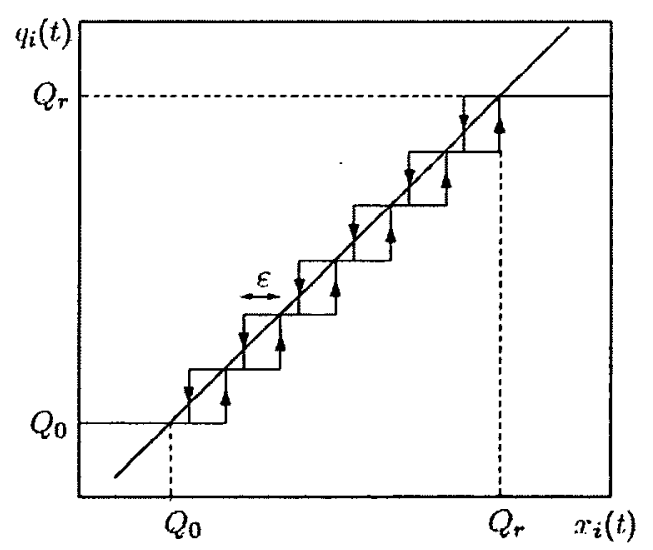

Figure 4: Quantization Function with Hysteresis (extracted, with permission, from [51]). 
A DEVS model that solves (Eq. 2.5) by integration is called a quantized integrator and can be written as follows [51]:

$$
\mathrm{M}_{1}=\left(\mathrm{X}, \mathrm{Y}, \mathrm{S}, \delta_{\text {int }}, \delta_{\text {ext }}, \lambda, \mathrm{ta}\right), \text { where: }
$$

$$
\begin{aligned}
& X=\mathfrak{R} \times\{\text { inport }\} \\
& Y=\mathfrak{R} \times\{\text { outport }\} \\
& S=\mathfrak{R}^{2} \times \mathrm{Z} \times \mathrm{R}_{0}^{+} \infty \\
& \delta_{\text {int }}(s)=\delta_{\text {int }}\left(x, d_{x}, k, \sigma\right)=\left(x+\sigma \cdot d_{x}, d_{x}, k+\operatorname{sgn}\left(d_{x}\right), \sigma_{1}\right) \\
& \delta_{\text {ext }}\left(s, e, x_{u}\right)=\delta_{\text {ext }}\left(x, d_{x}, k, \sigma, e, x_{v}, \text { port }\right)=\left(x+e \cdot d_{x}, x_{v}, k, \sigma_{2}\right) \\
& \lambda(s)=\lambda\left(x, d_{x}, k, \sigma\right)=\left(Q_{k+\varepsilon g n(d x)}, \text { outport }\right) \\
& \operatorname{ta}(s)=\operatorname{ta}\left(x, d_{x}, k, \sigma\right)=\sigma
\end{aligned}
$$

Where :

$\mathrm{R}_{0}^{+} \infty$ : Are the positive real numbers

\{Inport\}: Set of Input ports.

\{Outport\}: Set of Output ports. 


$$
\begin{aligned}
& \sigma_{1}=\left\{\begin{array}{ll}
\frac{Q_{k+2}-\left(Q_{k+1}\right)}{d_{x}} & \text { if } d_{x}>0 \\
\frac{Q_{k}-\left(Q_{k-1}-\varepsilon\right)}{\left|d_{x}\right|} & \text { if } d_{x}<0 \\
\infty & \text { if } d_{x}=0
\end{array}\right. \text { and } \\
& \sigma_{2}= \begin{cases}\frac{Q_{k+1}-\left(x+e . d_{x}\right)}{x_{v}} & \text { if } x_{v}>0 \\
\frac{\left(x+e . d_{x}\right)-\left(Q_{k}-\varepsilon\right)}{\left|x_{v}\right|} & \text { if } x_{v}<0 \\
\infty & \text { if } x_{v}=0\end{cases}
\end{aligned}
$$

A static function $f(z 1, \ldots, z p)$ can be represented by the DEVS model:

$$
M_{2}=\left(X, Y, S, \delta_{\text {int, }} \delta_{\text {ext }} \lambda, t a\right), \text { where }
$$

$X=\mathfrak{R} \times\left\{\right.$ inport $_{1}, \ldots$, inport $\left._{\mathrm{p}}\right\}$

$Y=\Re \times\{$ outport $\}$

$S=\mathfrak{R}^{p} \times \mathfrak{R}_{0}^{+} \infty$

$\delta_{i m}(s)=\delta_{i m}\left(z_{l}, \ldots, z_{p,} \sigma\right)=\left(z_{l}, \ldots, z_{p}, \infty\right)$

$\delta_{e x}(s, e, x)=\delta_{e x t}\left(z_{1}, \ldots, z_{p}, \sigma, e, x_{v}\right.$, port $)=$

$$
\left(z_{l}, \ldots, \tilde{z}_{p}, \infty\right)
$$

$\lambda(s)=\lambda\left(z_{1}, \ldots, z_{p}, \sigma\right)=\left(f\left(z_{1}, \ldots, z_{p}, \sigma\right)\right.$, outport $)$

$\operatorname{ta}(s)=\operatorname{ta}\left(z_{1}, \ldots, z_{p}, \sigma\right)=\sigma$

Where

$$
\widetilde{z}=\left\{\begin{array}{lr}
x_{v} & \text { if port }=\text { inport } \\
z_{j} & \text { otherwise }
\end{array}\right.
$$


As indicated in [51], this combined DEVS model of $M_{1}$ and $M_{2}$ simulates the QSS system.

Figure 5 shows a DEVS coupled model for a QSS model as defined by (Eq. 2.6) and (Eq. 2.7). The Model $M_{1}$ defines the integrator and the quantization function. This model has an input set $\mathrm{X}$ which contains pairs of (Real number, inport number), an output set $\mathrm{Y}$ that contains pairs (Real number, outport number), and a set of system states $\mathrm{S}$ that represents different states which the QSS system would have. Each state is represented with a tuple of the form $\left(x, d_{x}, k, \sigma\right)$ where $x$ is the state variable value, $d_{x}$ is the state variable rate of change (slope), $k$ is the index of the current quantization level $Q_{k}$, and $\sigma$ is the state lifetime.

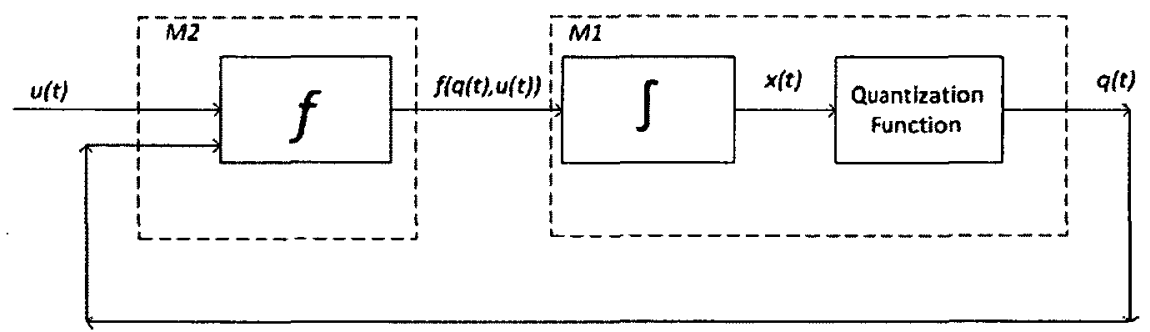

Figure 5: QSS Block Diagram Model

The behaviour of model $\mathrm{M}_{1}$ is determined by its transition functions $\delta_{\text {int }}(s)$ and $\delta_{\theta x x}(s, e$, $x)$. The function $\delta_{i t}(s)$ defines the internal transition function. This function transfers the system from its current state to a target state, and is executed when the state lifetime has elapsed. The target state of this function is always the next quantization level, which is expressed as the integration $\mathrm{x}+\sigma \cdot d_{x}=Q_{k+\operatorname{sign}(d x)}=Q_{k+1}$ (in case of a positive slope $d_{x}$ ). 
The lifetime of this target state $\sigma_{l}$ is the time needed to reach the next quantization level $\mathrm{Q}_{\mathrm{k}+2}$ and is calculated as shown above in (Eq. 2.6). $\delta_{\text {ext }}(S, e, x)$ defines the external transition function, and it is triggered whenever an input reaches an inport. In this model, the input is a pair $\left(x_{v}\right.$, port) where $x_{v}$ is the slope calculated as $x_{v}=f(q(t), u(t))$, as shown in the diagram of Figure 5. The target state of this function is a new state with an updated value of the state variable $x_{i+1}=x_{i}+e . d_{x}$, new slope $x_{v}$, and a new calculated lifetime $\sigma_{2}$ equals to the time needed to reach the next quantization level. $\lambda(s)$ in $M_{1}$ is the output function and when triggered, it sends the value of the current quantization level to the outport. $\mathrm{M}_{2}$ models a static function that accepts $q(t)$ and $u(t)$ as inputs and calculates $f(q(t), u(t)$.

An example of the quantization of an exponential decay function is shown below. The continuous representation of the function is shown in figure 6 , its linear approximation as defined by the QSS method is shown in figure 7, while its quantized representation is shown in figure 8.

In this example, the quantum (the difference between two successive quantization levels) is taken to be $\mathrm{dQ}=1$, and a set $\mathrm{Q}$ has 11 quantization levels $\mathrm{Q}=$ $\{0,1,2,3,4,5,6,7,8,9,10\}$. The system starts with an initial state variable value of 10 . As formally described in (Eq. 2.6) and (Eq. 2.7) the QSS approximates the continuous function with linear segments, each segment extends between two consecutive quantization levels. Whenever this approximation crosses a quantization level, the output function is triggered and sends an event containing the current value of this quantization 
level. The linear approximation to the decay graph is shown in Figure 7. The discrete levels generated by this linear approximation, through QSS model, are shown as constant segments in figure 8. Details of QSS model and solution for this function will be discussed in more detail in chapter 4.

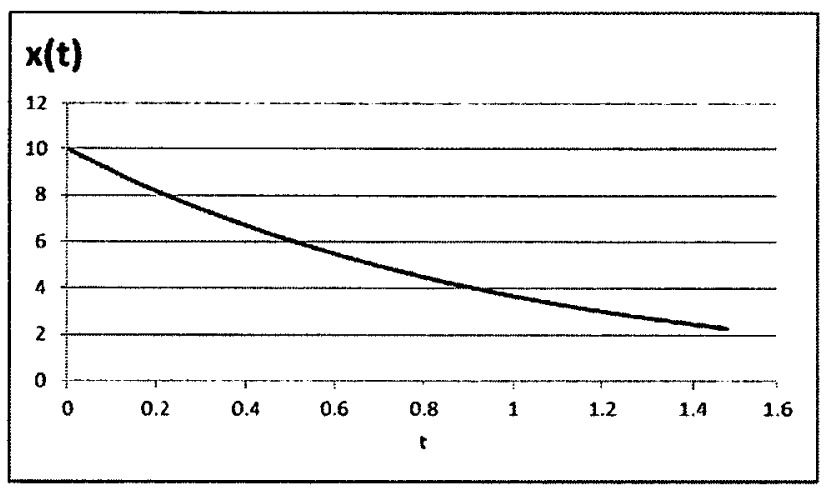

Figure 6: Continuous function of exponential Decay

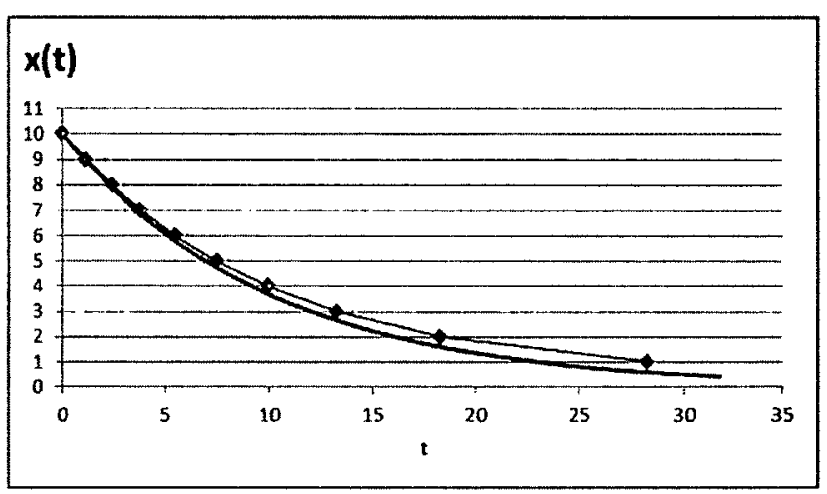

Figure 7: Linear approximation of Decay formula 


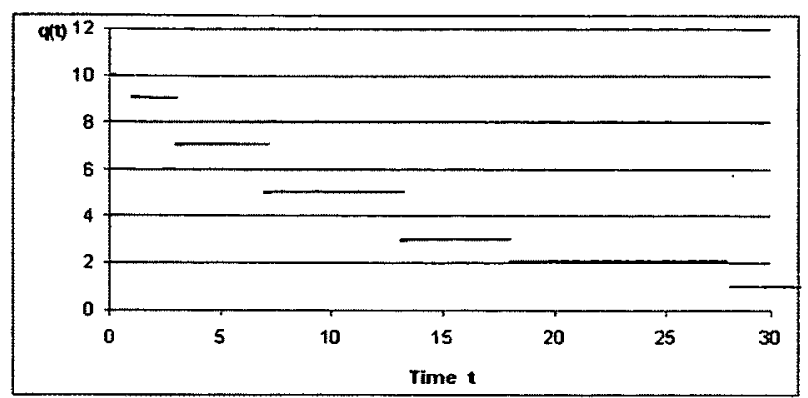

Figure 8: Quantized representation of Exponential Decay

\subsection{Introduction to Interval Arithmetic}

In many scientific measurements and computations, getting an exact answer is impossible. This is due to either uncertainty with measuring a physical value, or simply because some mathematical quantities cannot be expressed with the finite floating point representation in modern digital computers. Examples of this are rational numbers like $\sqrt{2}$ or $\pi$. In this case, the computer representation of this value would be an approximation. The difference between this approximation and the exact value is a roundoff error. Performing arithmetic computations with these approximated values produces an approximated answer as well. In many applications, scientists and engineers like to know some bounds on the roundoff error. This led to the development of interval arithmetic as a way to say the true answer is somewhere between two numbers $a, b$ which constitute a closed interval $[a, b]$ where $a \leq b[62]$. An interval $[a, b]$ is a set $\{x \mid x \in \mathscr{R}, a$ $\leq x \leq b\}$. For example, we may calculate $\sqrt{2}=1.4142135623730950488016887242097$ with 31 decimal digits. However, if we have an application with a precision of only 2 
decimal digits, we may get an answer of 1.41 , or 1.42 depending on the way the calculator rounds the result down or up. In either way, we do not get the true answer, and this may not be acceptable for many applications. Using an interval to express the answer as $[1.41,1.42]$ guarantees the inclusion of the true answer.

Ordinary mathematical operators can be defined for intervals, for example if $A=[a, b]$, $B=[c, d]$, then the following operations are defined for intervals [61] [62][63]:

$$
\begin{aligned}
& A+B=[a, b]+[c, d]=[a+c, b+d] \\
& \mathrm{A}-\mathrm{B}=[a, b]+[c, d]=[a-c, b-d] \\
& \mathrm{A} * \mathrm{~B}=[a, b] *[c, d]=\left[\min \left(a^{*} c, a^{*} d, b^{*} c, b^{*} d\right), \max \left(a^{*} c, a^{*} d, b^{*} c, b^{*} d\right)\right] \\
& \mathrm{A} / \mathrm{B}=[a, b] /[c, d]=[a, b] *[1 / d, 1 / c] \text { if } 0 \notin[c, d]
\end{aligned}
$$

If we know that $a, b, c, d$ are all positive numbers, then multiplication rule can be simplified as:

$$
\mathrm{A} * \mathrm{~B}=[a, b] *[c, d]=\left[\left(a^{*} c\right),\left(b^{*} d\right)\right]
$$

Arithmetic calculations for complex functions are also defined for intervals. There are about 18 different comparison operators for intervals, of which many do not exist for Real numbers. Moore [63] details many operations on interval arithmetic, properties of interval arithmetic, and computation algorithms for computing ODE solutions based on intervals.

This brief introduction of interval arithmetic is sufficient for our purposes for this thesis as details of this analysis is beyond the thesis scope. We will propose to use 
intervals and some of its arithmetic in section 5.1 for QSS verification as a way of bounding the true value calculated by QSS within some closed integer interval.

\subsection{Summary Discussion of the State-of-the-Art}

The approaches for the verification of DEVS that was covered during state-of-the-art survey have the following disadvantages when compared to the methodology introduced in this thesis:

1. Subclasses of DEVS (as SP-DEVS, FD-DEVS or TC-DEVS) constrain classic DEVS in such a way that it reduces expressiveness, and thus their usefulness to the system modeller. This limits what the modeller can do, and renders some portions of existing DEVS models to be non-verifiable, unless they are redefined to match the subclass definition (and only when this is possible).

2. The above subclasses of DEVS try to define their own reachability analysis by defining algorithms for building reachability graphs. These algorithms are based on basic model-checking algorithm, thus suffering from the same basic state-space explosion problem, and offer the same space-time complexity as standard model checking algorithms. These DEVS verification algorithms are implemented in ad-hoc model checking tools. Instead, there is a number of well-established model checking tools like UPPAAL and KRONOS, which have a large community of users and developers. These tools have implemented efficient data structures and optimizations that accumulated through years of research to optimize their performance. Nevertheless, such 
tools cannot verify DEVS subclasses with DEVS-specific reachability algorithms because unless DEVS subclasses are translated to TA, which is the input language of these tools, DEVS subclasses verification cannot be handled by these tools.

3. Some other approaches have translated DEVS models into another verifiable form such as TA, and then verified TA models by standard TA model checking tools like UPPAAL. These approaches, however, did not provide transformation based on formal behavioural equivalency between DEVS and TA. As a result, their transformation is not guaranteed to produce behaviourally equivalent TA for a given DEVS model.

4. Translation from DEVS to TA in previous approaches did not specify a method to translate DEVS models that may be unverifiable because it contains irrational values, and hence did not provide a method to estimate any effect of approximation irrational values on the verification results.

5. Previous translations approaches from DEVS to TA did not handle cases where DEVS model has continuous system components approximated with the QSS method. This excludes the hybrid systems from these verification approaches. 


\section{Chapter 3: Thesis contribution}

In order to deal with the multiple limitations of other approaches discussed in section 2.6, this thesis introduces a new set of methods for modeling, simulation and verification. The main objectives are the following:

1. Defining a methodology to translate models of DEVS to models of TA. This methodology can handle DEVS models that may contain irrational values, thus it could be used to verify any existing DEVS model without the need to re-write it with a different DEVS subclass.

2. The proposed approach translates DEVS to TA, through sound formal translation. This allows the use of standard tools like UPPAAL [9] to verify the resulting TA models. This approach takes advantage of numerous optimizations implemented in TA verification tools such as UPPAAL. TA was chosen for this research as it has strong theoretical background and mature verification tools such as UPPAAL and KRONOS. TA is also suited to state transition systems such as DEVS, while Timed Petri nets are suited more to process flow modeling [64]. The UPPAAL tool offers proven record of verification of industrial size problems with an easy interface that allows the user to enter the model and see the verification results.

3. The proposed approach uses a translation methodology that is based on bisimulation as a basis of behavioural equivalency between DEVS and TA. This 
equivalence relation is stronger than the trace equivalence [39], and it preserves all properties through the translation between the two models.

4. The approach provides a method to approximate any irrational values that may exist in a DEVS model. It also bases its reasoning on the effect of this approximation on the well-known results of robust TA [65][66][67] as we will show later. This gives the modeller a way to know if this approximation would affect the verification results for the given DEVS model or not.

5. The methodology extends existing verification methods to handle continuous systems modeled with DEVS through the QSS method. This enables the verification of hybrid DEVS models that contain discrete and continuous components.

At present, the methodology introduced in this thesis has achieved the goals above. This was done by first providing a definition of a new class of DEVS, called RTA-DEVS [68], which is very close to classic DEVS in semantics and expressive power. RTADEVS has followed FD-DEVS in restricting the time advance function to nonnegative rational numbers, but also relaxed the restriction of FD-DEVS on external transition functions. This makes RTA-DEVS closer to general DEVS than FD-DEVS. This enables the modeller to use a formalism that is more expressive than FD-DEVS to model a system and it is still formally verifiable.

As a second step, this thesis also discusses a transformation from RTA-DEVS models to TA models based on the bisimulation relation between the two models. This 
guarantees behavioural equivalence between the two models and preserves properties from RTA-DEVS into the resulting TA, and vice versa. This preservation is an important requirement to ensure that the verification results of TA are mapped back to RTA-DEVS models. Many classic DEVS models satisfy the semantics of RTA-DEVS models. Thus, they can be simulated with any DEVS simulator, and they can be transformed to TA to validate the desired properties formally.

As third step, we enable the verification of classic DEVS models that may contain irrational values and thus cannot directly be transformed to RTA-DEVS (and consequently to TA). To do so, this thesis proposed a method to transform DEVS to RTA-DEVS , and an approximation of DEVS models to RTA-DEVS as we showed in [68]. We used an approximation technique for irrational values to maintain the behaviour of the original DEVS model. In addition, we propose a method to estimate any effect the approximation may have on the final DEVS verification results [70][71].

Finally, a general method is proposed to model any QSS system with TA, thus enabling formal verification to continuous components modeled with the QSS method. A full list of published contributions, resulting from this work, is shown in the next section.

In Figure 9, we show the thesis contribution on a diagram representing expressive power and decidability. In this diagram, different formalisms are shown as ellipses. The containment relationship between a larger ellipse and a smaller one indicates that the expressiveness of the small is less than the larger one. In this diagram, we show some of DEVS subclasses (SP-DEVS, FD-DEVS and RTA-DEVS). These subclasses are less 
expressive than TA. However, TA formalism and these DEVS subclasses lie inside the boundary of decidable reachability. As SP-DEVS and FD-DEVS expressive power is less than DEVS, we had the chance to insert RTA-DEVS between these classes and DEVS, thus having more expressive power than SP-DEVS and FD-DEVS. The thesis contribution focuses on moving the verification problem from DEVS undecidable reachability domain, to the TA decidable reachability domain, while preserving reachability and safety properties. The ellipses with solid lines represent known results of expressive power. The dotted line between RTA-DEVS and TA indicated that RTADEVS expressive power is less than TA as evident by the work of this thesis; however the other direction of expressing TA models with RTA-DEVS was out-of-scope of this thesis.

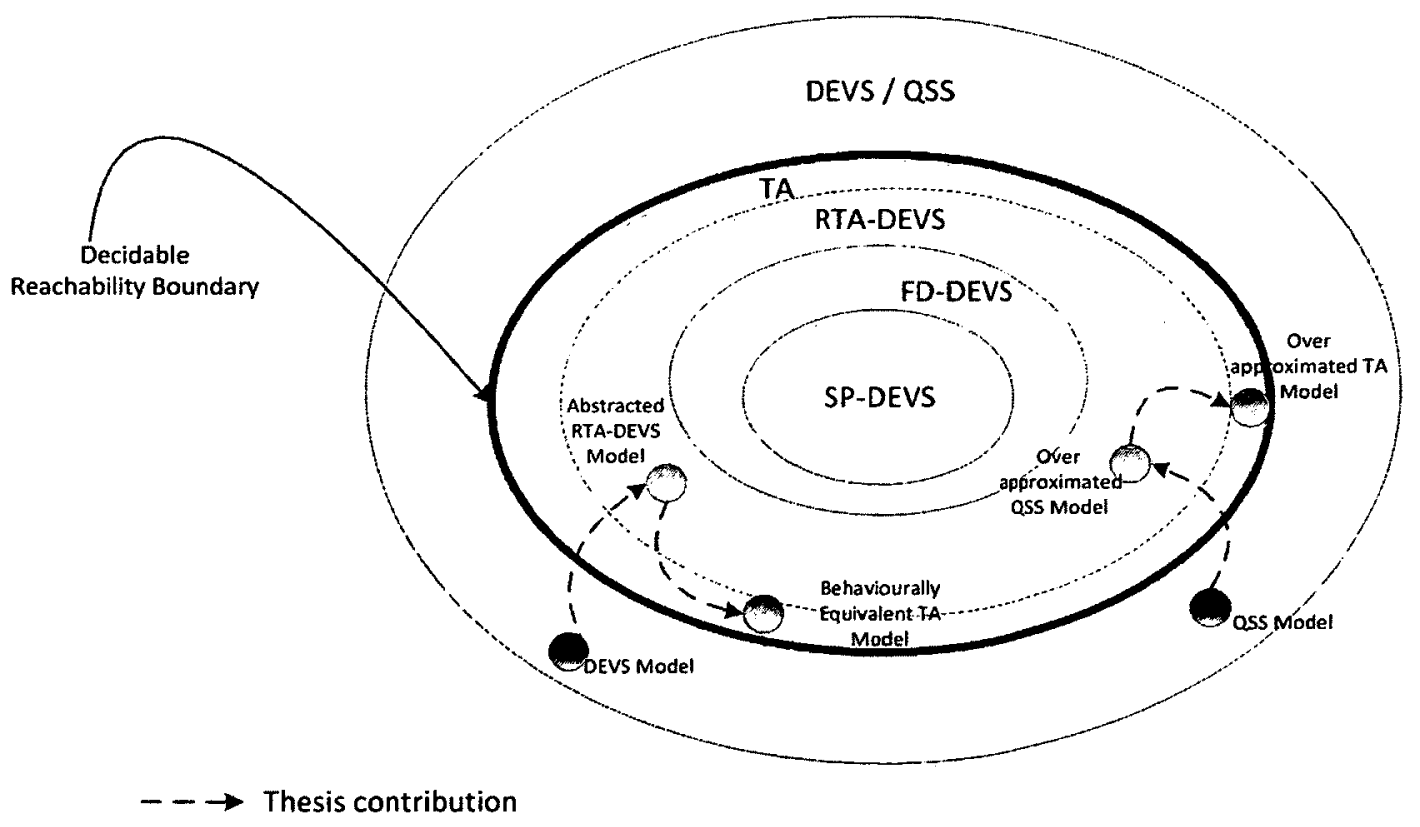

Figure 9: Thesis Contribution 


\subsection{List of Published Contributions}

- Hesham Saadawi, Gabriel A. Wainer. 2009. "Verification of real-time DEVS models". Proceedings of DEVS Symposium 2009. San Diego, CA [72]. In this paper, we introduced a methodology for verifying Real-Time DEVS models. Our methodology applies recent advances in theoretical model checking to DEVS models. The methodology also handles the cases where theoretical approach is not feasible to cross the gap between abstract Timed Automata models and the complexity of the DEVS Real-time implementation by empirical software engineering methods. This contribution is described in chapter 4 .

- Hesham Saadawi, Gabriel A. Wainer. 2010. "Rational time-advance DEVS (RTA-DEVS)". Proceedings of DEVS Symposium 2010. Orlando, FL. 2010. This paper was selected as the runner-up best paper in the Symposium. In this paper, we introduced a new extension to the DEVS formalism, called Rational TimeAdvance DEVS (RTA-DEVS). The basic idea of this new formalism is to permit modeling the behavior of systems that can be modeled by classical DEVS; however, RTA-DEVS models could be formally checked with standard modelchecking algorithms and tools. In order to do so, we introduce a procedure to create Timed Automata models that are behaviorally equivalent to the original RTA-DEVS models [68]. This contribution is described in chapter 4 . 
- Hesham Saadawi, Gabriel A. Wainer. 2010. "From DEVS to RTA-DEVS". In Proceedings of the 2010 IEEE/ACM 14th International Symposium on Distributed Simulation and Real Time Applications (DS-RT '10). IEEE Computer Society, Washington, DC, USA, 207-210 [73]. In this paper we defined the RTADEVS formalism to model and verify real-time embedded systems. In order to enable the formal verification of DEVS models, we introduced a procedure to approximate DEVS with RTA-DEVS. We also included the conditions for the valid approximated RTA-DEVS models and a calculation method for approximation errors that may be introduced. This contribution is described in chapter 4 .

- Hesham Saadawi, Gabriel A. Wainer. 2011. "Principles of DEVS Models Verification". Accepted for publication in: SIMULATION: Transactions of the Society for Modeling and Simulation International [70]. In this journal paper, we introduced our methodology to verify real-time embedded systems modeled with DEVS formalism. This is composed of a procedure to create Timed Automata models that are behaviorally equivalent to the original RTA-DEVS models, then, we described the use of the available TA tools and theories for formal model checking. Further, we introduced a methodology to transform classic DEVS models to RTA-DEVS, thus enabling formal verification of classic DEVS with an acceptable accuracy. This contribution is described in chapter 4 . 
- Hesham Saadawi, Gabriel A. Wainer, Mohammad Moallemi. 2012. "Principles of DEVS Models Verification for Real-Time Embedded Applications". Real-Time Simulation Technologies: Principles, Methodologies, and Applications. K. Popovici, P. Mosterman Eds. Taylor and Francis. CRC Press. 2012 [71]. In this book chapter, we explained our proposed methodology to verify real-time DEVS models of embedded systems. A case study of mechanical robot and its controller was defined and verified using our methodology. This contribution is described in chapter 4 .

- Hesham Saadawi, Gabriel Wainer. 2012. "On the verification of hybrid DEVS models". In Proceedings of the 2012 Symposium on Theory of Modeling and Simulation - DEVS Integrative M\&S Symposium (TMS/DEVS '12), Orlando, FL,USA. March 26-28. In this paper [81], we introduced a new verification methodology, based on RTA-DEVS, Timed Automata and the QSS method, which allows verifying real-time hybrid systems modeled by DEVS formalism. This contribution is described in chapter 5 .

- Hesham Saadawi, Gabriel Wainer. 2013. "Hybrid Systems Modeling and Verification with DEVS". In Proceedings of the 2013 Symposium on Theory of Modeling and Simulation - DEVS Integrative M\&S Symposium (TMS/DEVS '13), San Diego, CA, USA. April 7-10. Submitted for publication. In this paper, we introduce a methodology to verify hybrid systems modeled with DEVS and using 
general QSS method to model the continuous components. This contribution is described in chapter 5 . 


\section{Chapter 4: The RTA-DEVS formalism}

In chapter 1 and chapter 2, we have discussed the issues that are faced by the developers of RT embedded systems, and we gave a brief background about current techniques and formalisms that we plan to use to solve some of these issues. To accomplish the objective stated in chapter 1 , we need a simulation technique that is formally verifiable and has executable models. The latter is a property of Discrete-Event Systems Specification (DEVS) formalism [7], namely the facility to generate executable models from a discreteevent specification. For DEVS to be also verifiable, this thesis proposes a complete methodology to verify different types of DEVS, thus enabling full life cycle of RT systems development using DEVS. This development life cycle can be described with traditional software development cycle where phases of analysis, design can use formal verification as part of its activities. The verification methodology proposed here is based on a technique of formal software verification called model checking [19]. The advantage of this technique is that it is fully automated and does not need human intervention to reveal errors in system design.

As discussed earlier, the reason for introducing a new DEVS verification methodology is that existing methods limit verification to constrained subclasses of DEVS. This prevents the verification of wide range of existing DEVS models, and limits the modeller to use less expressive subclasses. In addition, the verification algorithms proposed for 
these subclasses need specially written verification tools. However, these specially written verification tools may not add much value over standard timed model checking tools. This is because these algorithms have the same time and space complexity as those of timed model checking algorithms, and thus DEVS special verification tools do not provide any advantages over timed model checking verification. On the other hand, verification tools for timed model checking are widespread and they usually contain many performance optimizations [25].

In order to do formal verification of DEVS, the methodology proposed is comprised of a few steps. The first step is to introduce a new formalism based on DEVS, but restricted in its behaviour to be less than DEVS to be verifiable. The reason why we use a restricted class of DEVS as a first step, is that the formal verification of general classic DEVS is undecidable as Hernandez and Giambiasi shown in [27] (and we would discuss this in more detail later). Creating a restricted subclass of DEVS is a way to enforce some restrictions into the model that can be created according to this subclass. This would guarantee the ability to verify any model belonging to this restricted DEVS subclass. We decided to use the model checking method due to its advantages over other formal software verification methods such as deductive methods. As we discussed in chapter 1 , the main advantage of model checking is that it is fully automated without human intervention, thus, it does not need advanced expertise in formal methods or formal proofs as opposed to deductive formal methods. As DEVS is a timed formalism (i.e. transition functions defining DEVS behaviour contain time in their domain), timed model 
checking was chosen to verify the proposed subclass of DEVS called Rational-Time Advance DEVS (RTA-DEVS) as published in [68] and we describe in this thesis in chapter 4. RTA-DEVS has followed FD-DEVS [34] in restricting the time advance function to nonnegative rational numbers, but also relaxed the restriction of FD-DEVS on external transition functions. This makes RTA-DEVS more expressive than FD-DEVS. However, RTA-DEVS still restricts the model in a way to remove the obstacles to formal verification. This restriction enables having RTA-DEVS models which are verifiable with standard formal verification tools for timed systems. In this way, the introduction of RTA-DEVS can be seen as imposing restrictions on the type of DEVS models that can be verified.

The second step shows a transformation from RTA-DEVS to TA. TA formal verification is decidable, and there are academic and industrial tools implementing this verification. However, TA is not designed to simulate complex systems, the physical systems around the embedded software, or to be deployed as executable models on target platforms. Instead, there are various DEVS environments that allow these; consequently, DEVS has been used in various efforts to model and simulate systems in their design phase. The transformation proposed here is based on the notion of bisimulation between RTA-DEVS and TA, which gives behavioural equivalency between the two formalisms, and it guarantees that any property in the RTA-DEVS model is preserved in the transformed TA model. The results of this work were published in [68]. 
The third step in the methodology is to find a way to verify any DEVS models that their definitions may not be restricted to RTA-DEVS specifications. To do so, a transformation from DEVS to RTA-DEVS is presented to obtain verifiable RTA-DEVS models as published in [73]. In that transformation process, we need to obtain an approximation to the DEVS model. In order to do so in a safe way, we introduce a mechanism to avoid introducing defects into RTA-DEVS when transforming from DEVS [73]. However, a question arises about the validity of transformed RTA-DEVS verification results when we apply it to the original DEVS model. In other words: how much confidence do we have in these results and what effect the approximation may have on the verification results?

The fourth step is to answer the above questions using theoretical results of TA, and to apply this theory to the model under verification, in order to estimate any verification error that may result from the approximations. These results were shown in [73].

In the methodology defined in this thesis, we use timed model checking because of its advantages as a practical method in industrial systems. As mentioned earlier, one of these advantages is the full automation without a need from a practitioner to be fluent in formal method or mathematical proofs. Some of widely used tools for TA models verification are UPPAAL [9] and KRONOS [74][75].

A final fifth step includes the use of a methodology to verify Continuous systems modeled by the Quantized Sate Systems (QSS) [51] method and DEVS. This would 
enable verification of hybrid systems (with discrete and continuous components modeled with DEVS).

With this, we provide a complete methodology that covers discrete and continuous DEVS components that can enable the formal verification of DEVS models. DEVS has the advantages of being based on a formal definition, with component-based hierarchical model construction, and is platform-independent. DEVS models are simulated within a DEVS simulator, which isolates the model from the implementation. This gives the advantage of avoiding any error that may creep between the steps of model verification and implementation by executing the verified model directly without mapping it to an executable code. This methodology gives the designer a comprehensive road map to verify different classes of DEVS models using standard formal verification tools.

The following sections explain the RTA-DEVS definition, its transformation to TA using the bisimulation relation to guarantee behavioural equivalence, the transformation of any classic DEVS model into RTA-DEVS, and the estimation of any effect that this last transformation may have on the verification results of RTA-DEVS. Verification of QSS models are described in chapter 5 .

\subsection{Rational Time Advance DEVS (RTA-DEVS)}

In [68], we proposed the RTA-DEVS formalism, a verifiable subclass of DEVS, with very much of the expressive power of DEVS. The difference in expressive power comes from the fact that RTA-DEVS does not accept irrational constants in its transition functions. However, as we see later, this difference in behaviour can be easily 
approximated to any desired precision in RTA-DEVS and we present a method by which we can conclude if there is any effect on verification results from this approximation.

As in classical DEVS, we define RTA-DEVS atomic model. The Atomic Rational Time-Advance is defined as:

$$
\mathrm{AM}_{\mathrm{TC}}=\left\langle X, Y, S, \delta_{i n s} \delta_{c x t}, \lambda, t a>\right.
$$

Where:

$-X$ : The set of external inputs.

- $Y$ : The set of external outputs.

- $S$ : set of system states.

- $\delta_{\text {int }}: S \rightarrow S$ is the internal transition function (the same as in classic DEVS).

- $\delta_{e x t}: T \times X \rightarrow S$ with $T=\left\{(s, e) \mid s \in S, 0 \leq e \leq t a(s), e \in Q_{0,+\infty}\right\}$ is the external transition function ( $e$ is the time elapsed since the last transition, which takes a positive rational value).

$-\lambda: S \rightarrow Y \cup \varnothing$ is the output function.

- ta: $S \rightarrow Q_{0,+\infty}$ is the time advance function that maps each state to a positive rational number.

RTA-DEVS changes the definitions of time advance function $t a$ and the external transition function $\delta_{e x t}$ from classic DEVS by removing the irrational time values. Other definitions stay the same, thus giving RTA-DEVS very close expressiveness to DEVS. A Coupled RTA-DEVS model is defined exactly as in classic DEVS. Coupled RTADEVS models are composed of any number of atomic or coupled RTA-DEVS models: 


$$
\mathrm{CM} \equiv\left\langle X, Y, D,\left\{M_{i}\right\}, C_{x}, C_{y}, \text { Select }\right\rangle
$$

Where:

$X$ : Set of external input events.

$Y$ : Set of external output events.

$D$ : Finite index of sub-components.

$\left\{M_{i}\right\}$ : The set of sub-components. A sub-component may be an atomic or coupled. $i \in D$ is the index of the component.

$C_{x}:$ Set of input couplings.

$C_{y}:$ Set of output couplings.

Select: $2^{D} \rightarrow D$ is a tie-breaking function, which defines how to select an event from a set of simultaneous events.

A coupled RTA-DEVS model $M$ can be simulated with an equivalent atomic RTADEVS model, whose behavior is defined as follows [76]:

$$
M=<X, Y, S, s_{0}, \delta_{i n t}, \delta_{\text {ext }}, \lambda, t a>
$$

Where $X$ and $Y$ are the input and output event sets, respectively. $X$ is the set of all input events accepted and $Y$ is the set of all output events generated by coupled model $M$. $S=X_{i \in D} V_{i}$ is the model's state. It is expressed as the Cartesian product of all the component's states, where $V_{i}$ is the total state for component $i$, $V_{i}=\left\{\left(s_{i}, t_{e i}\right) \mid s_{i} \in S_{i}, t_{e i} \in\left[0, \operatorname{ta}\left(s_{i}\right)\right]\right\}$. Here, $t_{e i}$ denotes the elapsed time in state $s_{i}$ of component $i$, and $S_{i}$ is the set of states of component $i$. 
$S_{0}=X_{i \in D} V_{0 i}$ the initial system state, with $V_{0 i}=\left(s_{0 i}, 0\right)$ is the initial state of component $i \in D$.

ta: $S \rightarrow T$ is the time advance function. It is calculated for the global state $s \in S$ of the coupled model, as the minimum time remaining for any state among all components. Formally: $\operatorname{ta}(s)=\min \left\{\left(\operatorname{ta}\left(s_{i}\right)-t_{e i}\right) \mid i \in D\right\}$ where $s=\left(\ldots,\left(s_{i}, t_{e}\right), \ldots.\right)$ is the global total state of coupled model at some point in time, $s_{i}$ is the state of component $i$, and $t_{e i}$ is elapsed time in that state.

$\delta_{\text {ext }}: X \times V \rightarrow S$ is the external transition function for the coupled model. Where $V$ is total state of the coupled model: $V=\left\{\left(s, t_{e}\right) \mid s \in S, t_{e} \in[0, t a(s)]\right\}$.

$\delta_{\text {int }}: S \rightarrow S \quad$ is the internal transition function of the coupled model.

$\lambda: S \rightarrow Y \quad$ is the output function of the coupled model.

In the following sections, we discuss RTA-DEVS and the mechanism to obtain a DEVS subclass, which removes difficulties to formal verification. Some of the most important of these difficulties is the irrational time values that could be defined in internal transition and external transition functions. RTA-DEVS restricts its functions definitions to the rational values, thus any valid RTA-DEVS model would be verifiable, as we have shown in [68]. 


\subsection{Estimate of Expressiveness Difference between DEVS and RTA- DEVS}

There have been many proposed DEVS subclasses we shown in section 2.3. These classes restricted the original DEVS behaviour in order to achieve decidable reachability. In doing so, some of the expressive power of original DEVS is lost. Although we cannot quantify how much expressive power is lost, we can devise a relative measure for how far the expressive power of a DEVS subclass from the original DEVS. If we look at the diagram shown in Figure 9, we observe that the wider the gap between the subclass and the DEVS, the more subclasses can be inserted in this gap. Therefore, we can take an relative measure of the gap width, from the ability to insert a number of DEVS subclasses in this gap. For example, SP-DEVS has much less expressive power than DEVS. This is evident by the fact that two other DEVS subclasses (FD-DEVS and RTA-DEVS) can be inserted between SP-DEVS and DEVS in the expressiveness diagram of Figure 9. Thus, a DEVS subclass " $X$ " is closest to DEVS expressiveness when no other subclass " $Y$ " can be inserted in the gap between $\mathrm{X}$ and the original DEVS. We claim this is the case here

for RTA-DEVS as it is the closest DEVS subclass in its expressive power, and still has decidable reachability.

Theorem: RTA-DEVS is the closest expressive and verifiable subclass to DEVS.

Proof:

We use induction to prove the above theorem. Assume there is another DEVS subclass, we call it X-DEVS, that is more expressive than RTA-DEVS and its 
reachability is decidable. From RTA-DEVS definition, we know that $\operatorname{ta}(s)=\sigma$ such that $\sigma_{\mathrm{RTA} \text {-DEVS }} \in\left\{Q_{0,+\infty}\right\}$, where $Q_{0,+\infty}$ is the set of positive rational numbers. Also from DEVS definition we have $\sigma_{\mathrm{DEVS}} \in\{\Re\}$, i.e. $\sigma_{\mathrm{DEVS}} \in\left\{Q_{0,+\infty} \cup \mathrm{IR}_{0,+\infty}\right\}$ where IR is the set of positive irrational numbers: IR $=\{\sqrt{2}, \sqrt{ } 3, \sqrt{5}, \pi, \ldots\}$. To define X-DEVS to be more expressive than RTA-DEVS, we extend its set of possible values of $\sigma$ to be more than RTA-DEVS. Any possible such extension to RTA-DEVS would have $\sigma_{X}$-DEVS $\in\{$ $\left.Q_{0,+\infty} \cup\left\{x \mid x \in \mathbb{I R}_{0,+\infty}\right\}\right\}$. The least possible extension would be with only one element from the set IR, i.e. $\sigma_{X-D E V S} \in\left\{Q_{0,+\infty} \cup\{\sqrt{2}\}\right\}$. However, this makes reachability analysis of this subclass of DEVS undecidable as it can have an irrational value in its time advance function domain. This contradicts our assumption above, therefore we conclude that we cannot have any subclass of DEVS which is more expressive than RTADEVS and still has a decidable reachability.

\subsection{RTA-DEVS to Timed Automata Behavioural Equivalence.}

To verify RTA-DEVS by applying existing theories and the tools for TA, a TA model must be constructed from the RTA-DEVS model in a way that guarantees its behavioural equivalency. DEVS, RTA-DEVS, and TA are all instances of Timed Labelled Transition Systems TLTS, and thus we would use here the methods of TLTS behavioural equivalence to move from one modelling formalism to another. We start our discussion here with a special case of TLTS which is the untimed LTS, and then we generalize our findings to TLTS. 
Generally there are two methods to check this (i.e., to see if two Labelled Transition Systems (LTS) are behaviourally equivalent), namely Trace Equivalence and Bisimulation. In [39], it was shown that, for RT Systems, trace equivalence is not enough to show the complete equivalence of two LTS. Although one can show the trace equivalency of two LTS (based on their acceptance or the generation of event traces), RTS usually have multiple concurrent components working together. Those components may go into a deadlock state in which no external event is observable. To show this, we will use an example that was introduced in [39] with some modifications. In this example, a user is interacting with an automated beverage machine that takes a coin and serves either coffee or tea. A LTS diagram representing the machine behaviour is shown in figure 10 . The machine CTM1 shown in this diagram waits at state PO for a coin to be inserted, then after coin insertion, it moves to state P1 to give the user a selection of tea or coffee. After serving the beverage, the machine returns to the waiting state P0. The behaviour of a user who always requires coffee can be represented in the LTS diagram in figure 11. This user puts the coin into the machine, and waits for the coffee to come out. We can see that CTM1 and the User would work together for the desired results of serving coffee. However, if we replace CTM1 with another machine with same observable behaviour (trace equivalent), we may replace it with something like CTM2 as shown in figure 12. Both CTM1 and CTM2 have the same observable trace for an outside observer, as both machines accept a coin and then dispense either tea or coffee. However if the user uses CTM2, it is possible to CTM2 to take the coin and then reach 
state P' 1 where it can only serve tea. In this case, the overall system composed of the User and CTM2 would reach a deadlock where the User waits for a coffee and the machine CTM2 cannot dispense it. This example shows that trace equivalence cannot reveal subtle errors such as this one. In a formal way to say this, trace equivalence preserves Linear Time Logic queries about the system. Queries with this logic cannot distinguish branches in the system behaviour. However, trace equivalence does not preserve Computation Tree Logic CTL that can distinguish between branches in system behaviour.

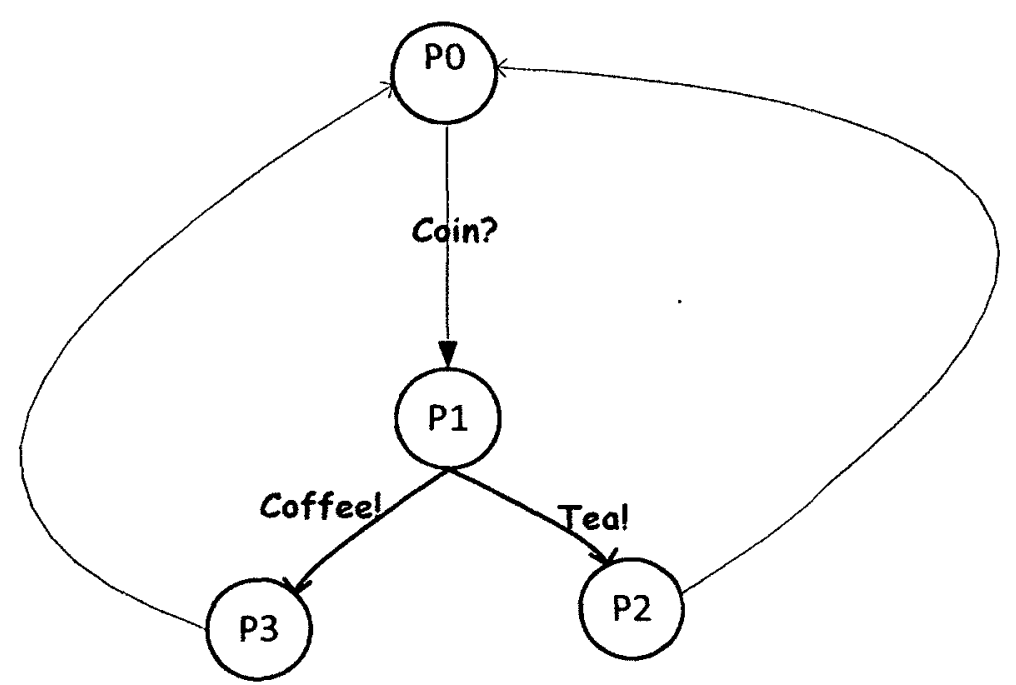

Figure 10: Coffee-Team-Machine 1 (CTM1). 


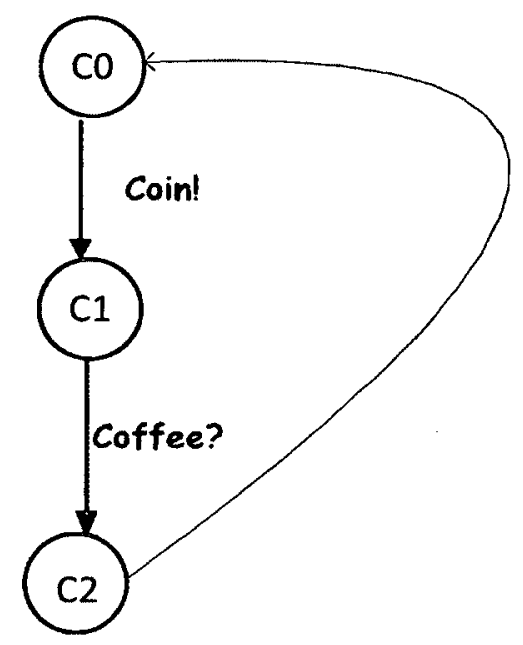

Figure 11: User Behaviour

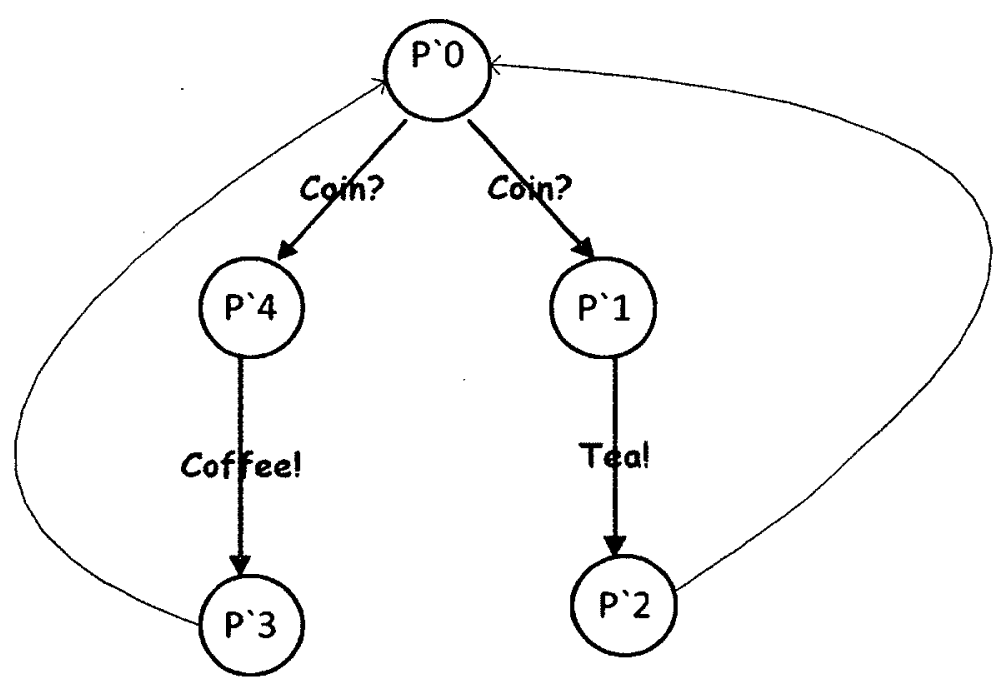

Figure 12: Coffee-Team-Machine 2 (CTM2).

Due to these subtle errors, bisimulation is a better notion for behaviour equivalence, and the same reason is valid for Timed LTS (TLTS), of which RTA-DEVS and TA are two examples. Formally, bisimulation preserves CTL query equivalence and thus can reveal such a subtle error in the example above. 
Bisimulation is a relation between two TLTS (e.g., systems A and B), which establishes a relation between every state in $\mathrm{A}$, and a corresponding one in $\mathrm{B}$. It also relates every observable transition in A to a corresponding one in B. In [39], the concepts of Strong and Weak timed bisimilarity were defined for behavioural equivalence of systems. In strong bisimulation, the two systems have to match in every state and transition. This condition may be too strong for a system that contains internal transitions with no observable actions to an outside observer. In this case, this system would not fulfill strong bisimilarity to another system that does not have unobservable. For this reason, the authors in [39] discussed the notion of a weak bisimulation, where unobservable transitions cannot affect behavioural equivalency as long as they begin and end between two bisimilar states. In this thesis, timed Weak bisimilarity equivalence was used, as it is more general in its application than the conditions for strong bisimulation. Contrary to strong bisimulation, weak timed bisimulation allows definition of behavioural equivalence relation between two systems even if they differ on some transitions or number of states. This gives the transformation from RTA-DEVS to TA greater flexibility to add or remove transitions or intermediate states in TA model that may not be in the original RTA-DEVS. This reduction in states allows the optimization for the model checking by reducing overall model size. Also, more optimization can be done by using certain features of UPPAAL TA like committed locations or urgent transitions even if these states has no direct match in the RTA-DEVS model. 
An example of this is shown in Figure 13. The model of (a) is an RTA-DEVS model where the system waits in state $\mathrm{S} 1$ for input a, then moves to state Aux 1. This state lifetime is 3 time units. After this time interval, the system moves to state Aux 2 with lifetime of 8 units, then moves to Aux3 and also after its lifetime interval elapses the system outputs $b$ and moves to state S5. The figure of (b) shows a TA model. This model is not strongly bisimilar to (a) as there are no states in (b) to simulate Aux1, Aux2, and Aux3. However, for our purpose of behavioural equivalence, these two models are equivalent by the weak bisimulation relationship as there is a weak bisimulation relation for states (S1, L1) and (S5, L3) that satisfies our concern of behavioural equivalence. This allows future enhancements to the transformation algorithms from RTA-DEVS to TA to reduce the number of states while preserving behavioural equivalence, thus reducing the resulting TA model size.

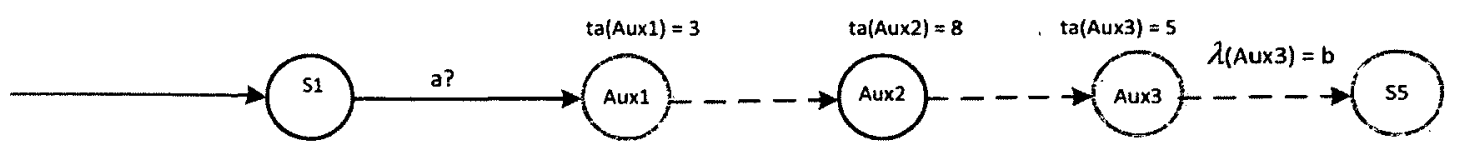

(a)

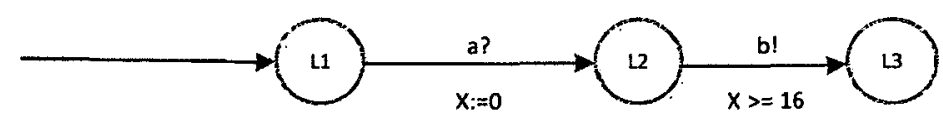

(b)

Figure 13: (a) RTA-DEVS model. (b) Timed Automata model 
In the following paragraphs, we define behaviour equivalency based on timed Weak bisimulation. Then, following the conditions of this bisimulation, a TA model for the basic behaviour elements of RTA-DEVS is constructed with some unknown constants in its guards and invariants. Then, we construct equations for these constants, based on the definitions of bisimulation, to determine the values of these constants.

Definition 2. Eventual transition relation ' $\Rightarrow$ ':

In a TLTS with states, $s$ and $t$, the eventual transition relation defines a transition from state $s$ to state $t$ that may contain one or more of direct transitions labelled with nonobservable events to the outside world. If we have an observable action $a$, a nonobservable action $\tau$, and a transition label $\alpha$, then, for any TLTS [39], the eventual transition relation $\Rightarrow$ between two states $s$ and $t$ on action $\alpha$ (written $s \stackrel{\alpha}{\Rightarrow} t$ ) is defined if any of the following conditions is true:

1. $\stackrel{\stackrel{\tau}{\Rightarrow} t}{\Rightarrow}$ : There is a transition from $s$ to $t$ only composed of transitions labelled with non-observable actions. E.g., for the non-observable action $\alpha=\tau$, there is a transition $s(\stackrel{\tau}{\longrightarrow})^{*} t$, (where $*$ defines one or more occurrences of these transitions).

2. $\stackrel{a}{s \Rightarrow t}$ : There is a transition from $s$ to $t$ composed of one transition labelled with an observable action $\alpha=a$, and one or more eventual transitions labelled with nonobservable actions. E.g., $\quad \stackrel{\tau}{s \Rightarrow} s_{1} \stackrel{a}{\longrightarrow} s_{2} \stackrel{\tau}{\Rightarrow} t$ for some states $s_{1}$ and $s_{2}$ 
3. $s \stackrel{d}{\longrightarrow} t:$ There is an eventual transition relation from $s$ to $t$ with total delay $d$ (called eventual delay transition), which is composed of one or more direct delay transitions combined with some non-observable action transitions. This represents a sequence of transitions with no observable actions whose total delay amounts to $d$. E.g., for action $\alpha=d \in \mathfrak{R}_{\geq 0}$ and $\stackrel{\tau}{s} s_{1} \stackrel{d_{1}}{\longrightarrow} t_{1} \ldots \ldots t_{n-1} \stackrel{\tau}{\Rightarrow} s_{n} \stackrel{d_{n}}{\longrightarrow} t_{n} \stackrel{\tau}{\Rightarrow} t$ (with $n \geq 0$ ), for some intermediate states $s_{l} \ldots s_{n}, t_{l} \ldots t_{n}$ and delays $d_{l} \ldots d_{n}$ with $d=\sum_{i=1}^{n} d_{i}$ (where $d$ is the total delay for the eventual transition from $s$ to $t$; by convention, $d=0$ when $n=0$ ).

Definition 3. Weak timed bisimulation:

The Weak timed bisimulation is a binary relation $\boldsymbol{R}$ over a set of states of a TLTS. For example if we have states $s_{1}, s_{1}^{\prime}, s_{2}$ and $s_{2}^{\prime}$, then $\boldsymbol{R}$ is a Weak timed bisimulation $s_{1} \boldsymbol{R} s_{2}$ [39] iff:

- $s_{1} \stackrel{d}{\longrightarrow} s_{1}^{\prime}$, then there is a transition $s_{2} \stackrel{d}{\Rightarrow} s_{2}^{\prime}$ such that $s_{1}^{\prime} \boldsymbol{R} s_{2}^{\prime}$ as shown in figure 14 .

- $s_{1} \stackrel{a}{\longrightarrow} s_{1}^{\prime}$, then there is a transition $s_{2} \stackrel{a}{\Rightarrow} s_{2}^{\prime}$ such that $s_{1}^{\prime} \boldsymbol{R}_{s_{2}^{\prime}}$ as shown in figure 15 .

- $s_{2} \stackrel{d}{\longrightarrow} s_{2}^{\prime}$, then there is a transition $\stackrel{d}{\stackrel{d}{\Rightarrow}} s_{1}^{\prime}$ such that $s_{1}^{\prime} R s_{2}$ as shown in figure 16.

- $s_{2} \stackrel{a}{\longrightarrow} s_{2}^{\prime}$, then there is a transition $s_{1} \stackrel{a}{\Rightarrow} s_{1}^{\prime}$ such that $s_{1}^{\prime} \boldsymbol{R} s_{2}^{\prime}$ as shown in figure 17 . 

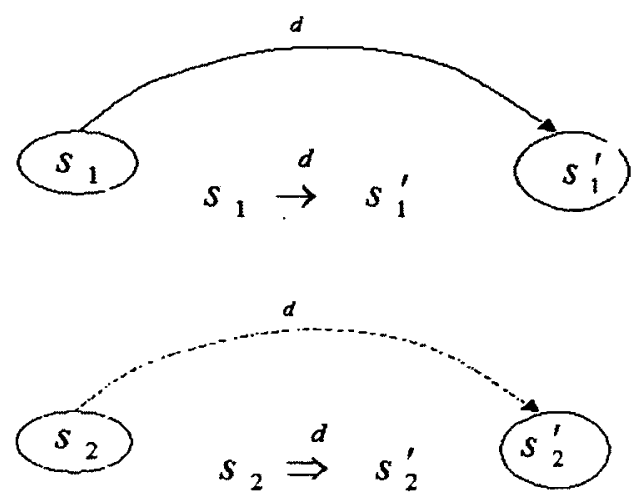

Figure 14: Direct delay transition from $s_{1}$ to $s_{1}^{\prime}$ and corresponding eventual delay transition from $s_{2}$ to $s_{2}^{\prime}$.

In the figures 14 to 17 , we express a state with oval shape, delay or action transition with an arrow, direct transition (i.e. single transition from one state to another) is represented with solid arrow, and an eventual transition is represented with dotted arrow.

We chose using the Weak bisimulation relation to transform from RTA-DEVS to TA and vice versa, as this relation allows two models to be in bisimulation relation even with if one of them has a different number of transitions or states from the other, provided that these extra transitions are labelled with non-observable action $\tau$ [39]. This relaxation over strong bisimulation allows more flexibility to tune the TA model for model checking performance while keeping the bisimulation relation to the RTA-DEVS model. 

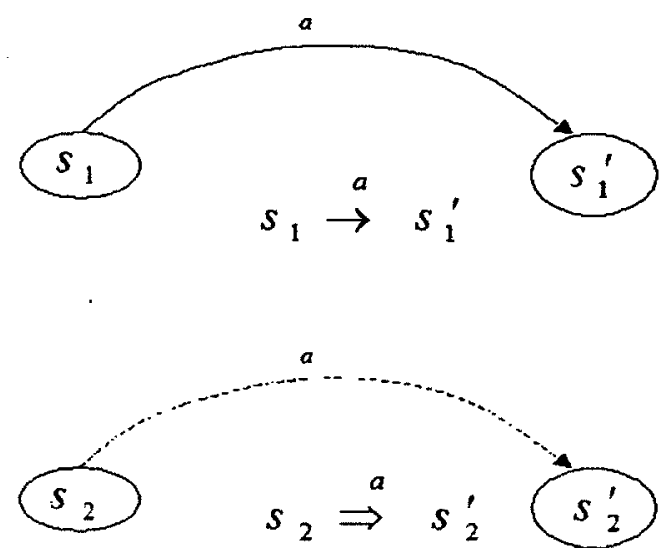

Figure 15: Direct action transition from $s_{1}$ to $s_{1}{ }_{1}$, and corresponding eventual action transition from $s_{2}$ to $s_{2}^{\prime}$.

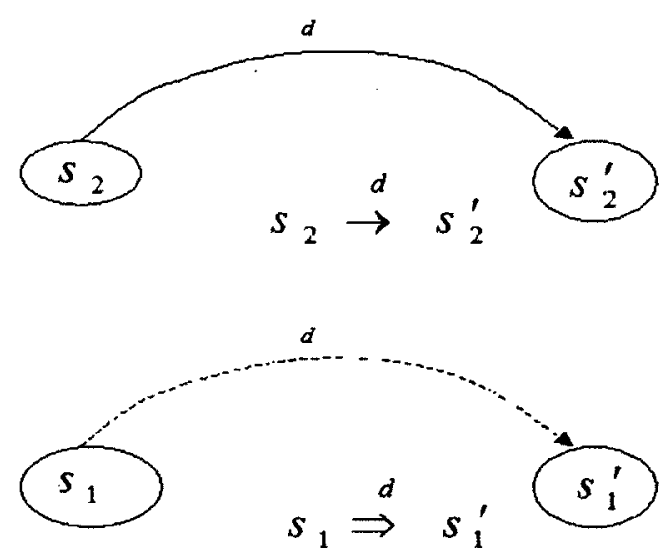

Figure 16: Direct delay transition from $s_{2}$ to $s_{2}^{\prime}$ and corresponding eventual delay transition from $s_{l}$ to $s_{l}^{\prime}$. 

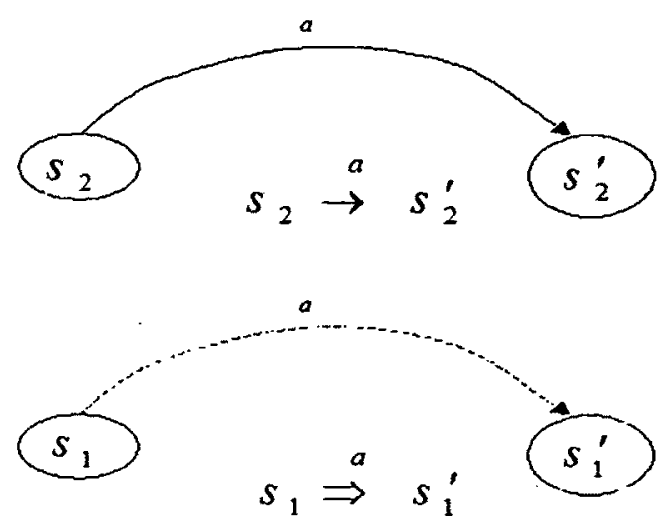

Figure 17: Direct action transition from $s_{2}$ to $s_{2}^{\prime}$, and corresponding eventual action transition from $s_{l}$ to $s_{\prime}^{\prime}$.

\subsubsection{RTA-DEVS internal transition semantics}

As shown in the previous section, TA expresses the notion of time through clock variables and constraints on them. Here, we present the RTA-DEVS behaviour in terms of its internal transition function, and then discuss how to obtain a behaviourally equivalent TA transition according to the previous definition of bisimulation.

Within TA semantics, there are two conditions for a transition to be enabled. These are the evaluation to true of the guard condition, and also the destination state invariant. . We apply these conditions here to derive the conditions we need for our transformation from RTA-DEVS to TA. The second condition, destination state invariant is true, is always satisfied within RTA-DEVS semantics. The following steps would work with the first condition of transition guard evaluating to true. 
By using these conditions to derive inequalities with the unknown constants, we determine the values of these constants in TA invariants and guards, in order to make TA model bisimilar to the RTA-DEVS model.

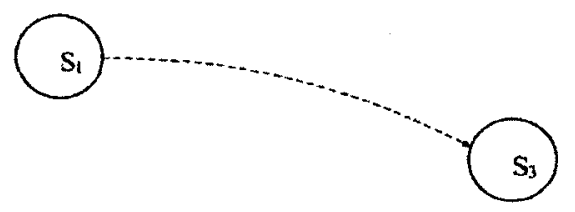

Figure 18: RTA-DEVS Internal Transition.

The RTA-DEVS internal transition semantics is shown as a DEVS graph in figure 18, and is defined as:

$$
\delta_{i n t}\left(s_{1}, e\right)=s_{3} \quad \text { if } \quad e=t a\left(s_{1}\right)=T
$$

In this semantics, this transition means that we move to state $s_{3}$ when the elapsed time $e$ in $s_{1}$ equals the time advance value of $s_{1}$ (which is $T$ ). In a TLTS, this can be defined as a time-elapse transition with delay $d$, assuming we start in state $s_{I}$ with elapsed time $e$, this can be expressed in the form:

$$
s_{1} \stackrel{d}{\longrightarrow} s_{3} \quad \text { if } 0 \leq e \leq t a\left(s_{1}\right) \text { and } d=t a\left(s_{1}\right)-e
$$

This means that if we start at $\mathrm{s} l$ with time spent in $s_{l}$ equals to $e$, we need to delay $d$ time units before changing to state $s_{3}$.

The other part of the semantic of the internal transition is that we stay in the same state as long as elapsed time $e$ does not reach the time advance value of this state $T$. This is expressed as:

$$
\delta_{\text {int }}\left(s_{1}, e\right)=s_{1} \quad \text { if } \quad 0 \leq e<\operatorname{ta}\left(s_{1}\right)
$$


This part of RTA-DEVS semantics can be defined for TLTS as a time-elapse transition on the form:

$$
s_{1} \stackrel{d}{\longrightarrow} s_{1} \quad \text { if } 0 \leq e \leq t a\left(s_{1}\right) \text { and } 0 \leq d<\operatorname{ta}\left(s_{1}\right)-e
$$

This means that if we start at $s_{1}$ with time spent $e$, as long as time delay $d$ is constrained as above, we stay at $s_{1}$.

In this thesis, we are using the operational semantics of UPPAAL TA as defined in [10]. For the graphs in the rest of the Thesis, RTA-DEVS states would be named as $s_{i}$, and the corresponding TA locations as $L_{i}$ (in which $i$ is an integer number).

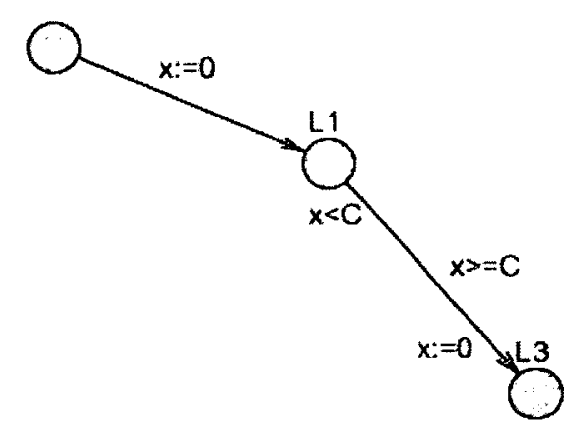

Figure 19: TA model for an Internal RTA-DEVS transition.

From the semantics of TA, the delay transition for the TA in figure 19 is defined as:

$$
\left(L_{1}, \text { clock }=t\right) \stackrel{d}{\longrightarrow}\left(L_{1}, \text { clock }=t+d\right) \quad, \forall \mathbf{0} \leq \mathbf{d}<\mathbf{C}
$$

For this to define the RTA-DEVS delay transition, it should stay in the $L_{l}$ state with a total delay $d$ less than the time advance of $s_{\mid}$. Our target is to obtain the TA in figure 19 to be behaviourally equivalent to the DEVS graph model shown in figure 18 through a 
timed bisimulation relation. To do this, we will show that the state $s_{1}$ is bisimilar to location $L_{l}$, and state $s_{3}$ is bisimilar to location $L_{3}$. This is done using the definition of the Weak timed bisimulation relation (from RTA-DEVS to TA, and from TA to RTADEVS). We do these in steps 1 and 2 below.

In this TA model, two locations are defined $\left(L_{1}\right.$ and $\left.L_{3}\right)$, along with a transition from $L_{l}$ to $L_{3} . L_{1}$ has an invariant on clock $x(x<C)$ that allows the TA to stay in that location as long as the invariant is true. The transition from $\mathrm{L}_{1}$ to $\mathrm{L}_{3}$ has a guard $(x \geq C)$ that must be true for the transition to be enabled, and $c$ is a rational number. The transition also has an update rule for clock variable $x$ to reset it to zero before entering location $\mathrm{L}_{3}$. By applying the condition above to weak timed bisimulation, first from RTA-DEVS to TA and then from TA to RTA-DEVS, a value for the constant $C$ to preserve the bisimulation relation can be determined

\section{Step1: from RTA-DEVS to TA}

For the bisimulation of the states shown in figure 18 and figure 19 , we have the following requirements:

- $s_{l} \boldsymbol{R} L_{l}$ : This is a delay transition from $\mathrm{s}_{1}$ to itself. If $s_{1} \stackrel{d}{\longrightarrow} s_{1}$ for some value of $d$ where $0 \leq e \leq t a\left(s_{1}\right)$ and $0 \leq d<t a\left(s_{1}\right)-e \quad$; then to satisfy the bisimulation relation we should have a transition in TA as: $\left(L_{1}, x=e\right) \stackrel{d}{\longrightarrow}\left(L_{1}, x=e+d\right)$ for the same value of $d$. Therefore from the invariant of state $\mathrm{L}_{1}$ we get $x<C$, then by substituting for $x$ and $d$, we get: 


$$
\operatorname{ta}\left(s_{1}\right) \leq C
$$

- $\quad s_{3} \boldsymbol{R} L_{3}$ : Bisimilarity between $\mathrm{s}_{3}$ and $L_{3}$ gives us the following:

For the delay transition from $s_{1}$ to $s_{3}$, let us consider the execution of the DEVS internal transition $\left(s_{1}, e\right) \stackrel{d}{\longrightarrow}\left(s_{3}, 0\right)$, where $0 \leq e \leq t a\left(\mathrm{~s}_{1}\right)$ and $d=\operatorname{ta}\left(s_{1}\right)$-e by the TA transition $\left(L_{1}, x=e\right) \stackrel{d}{\longrightarrow}\left(L_{3}, x=e+d\right)$, with the reset statement $x:=0$ on the transition.

In order for these two delay transitions to be equivalent, they need to start from bisimilar states, and after same amount of time delay, they reach two bisimilar states. To achieve this, the same value of delay $d$ is used on both transitions. From this we deduce the constant $C$ in the TA clock constraint to give the condition for bisimulation. The TA transition above starts from location $L_{l}$, with the clock $x$ valuation equals that of elapsed time $e$ at the RTA-DEVS transition above; then after delay $d$ of the RTA-DEVS transition, it transitions to $L_{3}$, and the value of clock $x$ increases by $d$.

By applying the conditions for this delay transition on TA transition above, we get (Eq. 4.6) from the TA guard $(x \geq C)$ and the valuation of $\operatorname{clock} x(x=e+d)$ :

$$
\operatorname{ta}\left(s_{1}\right) \geq C
$$


This constraint means that as long as we use a constant $C$ in the guard of the TA transition with some greater or equal value of the time advance of $s_{1}$, the previous TA transition simulates the RTA-DEVS transition above.

Combining (Eq. 4.5) \& (Eq. 4.6) gives the condition $t a\left(s_{1}\right)=C$ for the TA shown in figure 19 to execute and simulate the DEVS graph as in figure 18.

This condition guarantees the timed Weak simulation relation from the RTA-DEVS model internal transition of figure 18 to the delay transitions of TA in figure 19. Following this, we would show the condition of timed Weak simulation relation from TA to RTA-DEVS in step 2 below and this would complete the conditions for bisimulation between RTA-DEVS and TA.

\section{Step 2: From TA to RTA-DEVS}

To satisfy the other direction of the bisimulation relation, we convert the TA in figure 19 with RTA-DEVS in figure 18.

Case 1: TA delay transition $\left(L_{1}, x=e\right) \stackrel{d}{\longrightarrow}\left(L_{1}, x=e+d\right)$

Here, we need the value of clock $x$ to be less than $C$ in order for the $L_{l}$ invariant $(x<c)$ to be true and for the TA to stay in $L_{l}$, i.e.

$$
e+d<C
$$


For the RTA-DEVS time delay transition $\left(s_{1}, e\right) \stackrel{d}{\longrightarrow}\left(s_{1}, e+d\right)$ to stay in $s_{1}$ after $d$, we need the sum of the elapsed time and delay to be less than the lifetime of $s_{1}$, this is expressed as:

$$
e+d<\operatorname{ta}\left(s_{1}\right)
$$

Case 2: TA transition $\left(L_{1}, x=e\right) \stackrel{d}{\longrightarrow}\left(L_{3}, x=0\right)$.

This transition starts from location $L_{1}$ with a clock $x$ equal to some elapsed time $e$ in $L_{l}$. After delay $d$, TA transitions to $L_{3}$ and clock $x$ is reset. On exit from $L_{l}, L_{l}$ invariant would be false and the guard on the TA transition would be true, these give (Eq. 4.9) as follows:

$$
e+d=C
$$

This is defined in RTA-DEVS as $\left(s_{1}, e\right) \stackrel{d}{\longrightarrow}\left(s_{3}, 0\right)$, in which we need elapsed time $e$ in $s_{l}$ and a delay equal to the time advance of $s_{l}$ to trigger the internal transition:

$$
e+d=\operatorname{ta}\left(s_{1}\right)
$$

From (Eq. 4.7) and (Eq. 4.8), we can determine $c=t a\left(s_{1}\right)$. With this value, we showed a timed simulation relation from TA (in figure 19) to RTA-DEVS (in figure 18). By having a simulation relation in both directions, the RTA-DEVS internal transition shown above is timed bisimilar and behaviourally equivalent to the TA timed transitions shown above 
if we have the constant $c$ equals to the lifetime of the corresponding state in RTA-DEVS model. This concludes that $s_{1} \boldsymbol{R} L_{1}$ and $s_{3} \boldsymbol{R} L_{3}$ by the bisimulation relation $\boldsymbol{R}$.

When the previous method is used to map internal transitions from RTA-DEVS model to transitions at a TA model and vice versa, the resulting transitions are guaranteed to be behaviourally equivalent. We will show the same for RTA-DEVS external transitions in the following section.

\subsubsection{RTA-DEVS external transitions semantics}

The RTA-DEVS external transition function is defined as: $\delta_{e x t}: V_{D} \times X \rightarrow S$, where:

$$
V_{D}=\{(s, e): s \in S, 0 \leq e<t a(s)\}
$$

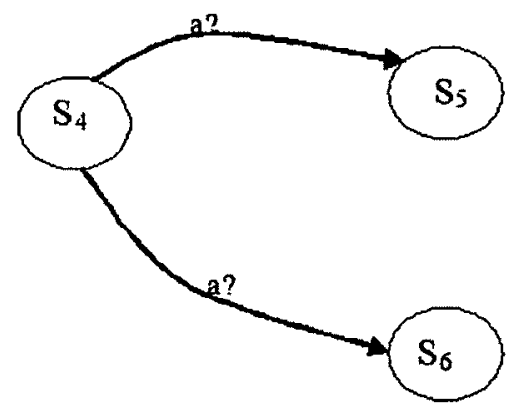

Figure 20: RTA-DEVS External transitions on action a.

Figure 20 represents the following definitions for the RTA-DEVS external transition function:

$$
\begin{array}{ll}
\delta_{e x t}\left(s_{4}, a, e\right)=s_{5} & \text { For } 0 \leq e<k \\
\delta_{e x(}\left(s_{4}, a, e\right)=s_{6} & \text { For } k \leq e<\operatorname{ta}\left(s_{4}\right)
\end{array}
$$

Each of these transitions can be expressed as a time passage and action transitions as: 
1. $s_{4} \stackrel{d<k}{\longrightarrow} s_{4}$ and $s_{4} \stackrel{a}{\longrightarrow} s_{5}$

2. $s_{4} \stackrel{k \leq d<t a\left(s_{4}\right)}{\longrightarrow} s_{4}$ and $s_{4} \stackrel{a}{\longrightarrow} s_{6}$

From these expressions, the external transitions can be represented as the TA transitions shown in figure 21 assuming $k=3$.

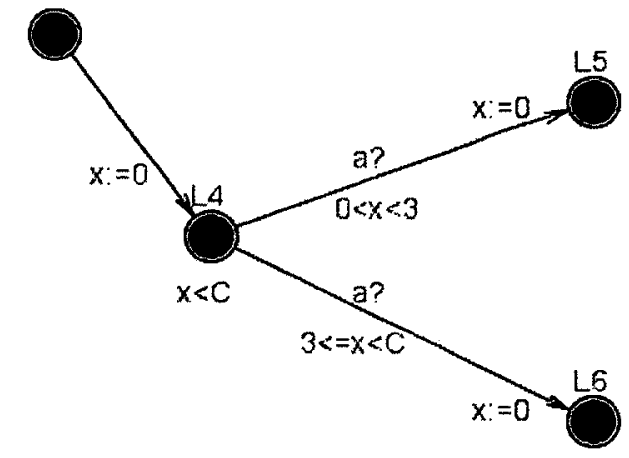

Figure 21: TA model for RTA-DEVS external transition.

From TA semantics, each of these transitions can be expressed as a time and action transitions as follows:

1. $\left(L_{4}, x=0\right) \stackrel{d<k}{\longrightarrow}\left(L_{4}, x=k\right)$ and $\left(L_{4}, x=k\right) \stackrel{a}{\longrightarrow}\left(L_{5}, x=0\right)$ for the first RTADEVS transition. That is, TA stays in $L_{4}$ while elapsed time is less than $k$ units, and then after this time elapses the TA takes a transition to $L_{5}$ with action $a$.

2. $\left(L_{4}, x=0\right) \stackrel{k \leq d<t a\left(s_{4}\right)}{\longrightarrow}\left(L_{4}, x=k\right)$ and $\left(L_{4}, x\right) \stackrel{a}{\longrightarrow}\left(L_{6}, x=0\right)$ for the second RTA-DEVS transition. That is, if the elapsed time in $L_{4}$ exceeds $k$ units and is less than the lifetime of $s_{4}$, the TA transitions to $L_{6}$ with action $a$. 
These give the relation $\boldsymbol{R}$ between RTA-DEVS and TA model states: $s_{4} \boldsymbol{R} L_{4}, s_{5} \boldsymbol{R} L_{5}$, and $s_{6} R L_{6}$.

Conversely, the simulation in the other direction from each of TA transitions to the RTA-DEVS external transitions above can be shown. Hence, this shows a bisimulation relation $\boldsymbol{R}$ between the corresponding DEVS and TA models above.

\subsubsection{An Alternative way to show bisimulation between RTA-DEVS Internal Transition and TA Transition.}

We may simplify the discussion in sections 4.3 .1 by assuming the value of time advance function $\operatorname{ta}\left(s_{1}\right)$ to be $C$ and then checking the existence of bisimulation between RTADEVS internal transition shown in Figure 18 and the TA transitions shown in Figure 19. In Figure 18, the semantics of RTA-DEVS internal transition gives a delay transition where the model stays in the same state as:

$$
s_{1} \stackrel{d}{\longrightarrow} s_{1} \quad, 0 \leq d<C
$$

This is simulated by the delay transition of TA in Figure 19 as:

$$
\left(L_{1}, x=0\right) \stackrel{d}{\longrightarrow}\left(L_{1}, x=d\right) \quad, 0 \leq d<C
$$

Also a delay transition of Figure 18 where we the model moves to another state as:

$$
s_{1} \stackrel{d}{\longrightarrow} s_{3} \quad, d \geq C
$$

Is simulated by the delay transition of TA in Figure 19 as: 


$$
\left(L_{1}, x=0\right) \stackrel{d}{\longrightarrow}\left(L_{3}, x=0\right), d \geq C
$$

We can also see the simulation relation in the other direction as TA transition of Figure 19 is simulated by RTA-DEVS transition of Figure 18. These two simulations establish a bisimulation relation between the transitions in the two figures.

\subsection{An algorithm for behavioural equivalence}

In our published works [68] [69], we introduced the following methodology to transform RTA-DEVS models to TA models.

1. Define a clock variable for each atomic RTA-DEVS model (i.e. $\operatorname{clock} x$ ).

2. For all constants defined in $t a\left(s_{i}\right)$, and $\delta_{\text {ext }}\left(s_{i}\right)$ if any rational numbers exist, convert the model scale to make them integers as shown in section 4.5.

3. Replace every state in RTA-DEVS with a corresponding location in TA (i.e. $L_{I}$ for source $s_{1}$ and $L_{2}$ for destination $s_{2}$ ).

4. Model the RTA-DEVS internal transition with TA as follows:

- Reset the clock variable on the entry to each state $(x:=0)$.

- A source state $L_{1}$ and a destination state $L_{2}$.

- Put an invariant in the source state derived from the time advance function for that state, i.e. $x<\operatorname{ta}\left(s_{l}\right)$.

- Define a transition with a guard. This guard should be the complement to the invariant in the source state, i.e. $x \geq \operatorname{ta}\left(s_{l}\right)$.

- Define an action for each output function defined. 
5. The RTA-DEVS external transition is modeled in TA with the following items:

- A source state and some destination state(s), i.e. $L_{l}$ for source $s_{l}$ and $L_{2}$ for destination $s_{2}$.

- A clock reset on the entry to each state.

- An invariant in the source state that corresponds to time advance function for that state, i.e. $x<t a\left(s_{1}\right)$.

- For the external transition(s) with guards of clock constraints, these constraints should be disjoint to obtain a deterministic TA model.

- The action label on TA transitions for each RTA-DEVS input event to source state $s_{l}$.

A formal definition of the algorithm is given as follows:

Transform_RTA-DEVS_To_TA(in RTA-DEVS, out TA)

\{

1. Declare a set of clocks $C=\left\{x_{i}|1 \leq i \leq| D \mid\right\}$, where $i$ is the index of component $d \in D$

2. Convert rational numbers defined in RTA-DEVS model to integers as described in section 4.5 .

3. Define a TA location for each RTA-DEVS state and define location invariant if necessary:

For each $d \in D$ do

$$
N_{d}=\left\{l_{j} \mid \exists s_{j} \in S_{d}, d \in D\right\} / / \text { component } N \text { of TA model corresponding to component d }
$$




$$
\beta(C)_{l j}=\left\{x_{i} \leq t a\left(s_{j}\right)\left|\exists s_{j} \in S_{d}, 1 \leq i \leq\right| D \mid, \operatorname{ta}\left(s_{j}\right)<\infty\right\}
$$

end do

4. Define a set of channels for communication between TA components:

$$
H=\left\{a_{j} \mid j \in I C\right\}
$$

5.Define set of TA transitions for RTA-DEVS internal transitions:

$$
\text { set } E=\varnothing ; \quad \quad \text { //Initialize the set of } T A \text { Transitions }
$$

For each $d \in D$ do

For each $s_{j} \in S_{d} d o$

$$
\begin{gathered}
I f\left(\operatorname{ta}\left(s_{j}\right)<\infty \& \& \delta_{i n t}\left(s_{j}\right)=s_{k} \& \& \lambda\left(s_{j}\right)=a\right) \text { then } \\
E=E \cup\left(l_{j}, x_{d} \geq \operatorname{ta}\left(s_{j}\right), a !, x_{d}:=0, l_{k}\right)
\end{gathered}
$$

end if

end do

end do

6. Add TA transitions corresponding to RTA-DEVS external transitions:

For each $d \in D$ do

$$
\begin{aligned}
& \text { For each } s_{j} \in S_{d} \text { do } \\
& \qquad \begin{array}{r}
\text { If }\left(\delta_{e x t}\left(s_{j}, a, \operatorname{cond}(e)_{m}\right)=s_{k m}\right) \text { then } \\
\left.E=E \cup\left(l_{j}, \operatorname{cond}\left(x_{d}\right)_{m}\right), a ?, x_{d}:=0, l_{k m}\right) ;
\end{array}
\end{aligned}
$$

end if

end do

end do 


$$
\begin{aligned}
& \text { I/ whenever: } \quad \delta_{\text {ext }}\left(s_{j}, a, e\right)=s_{k l} \quad \operatorname{cond}(\mathrm{e})_{1}: 0 \leq e<c_{l} \\
& / / \quad=s_{k 2} \quad \operatorname{cond}(\mathrm{e})_{2}: c_{1} \leq e<c_{2} \\
& / / \quad=s_{k m} \quad \operatorname{cond}(\mathrm{e})_{\mathrm{m}}: c_{m-1} \leq e<c_{m}
\end{aligned}
$$

// then we have define $\operatorname{cond}\left(x_{d}\right)_{m}=c_{m-1} \leq x_{d}<c_{m}$

$/ /$ where $\operatorname{cond}\left(x_{d}\right)_{1}, \operatorname{cond}\left(x_{d}\right)_{2}, \ldots, \operatorname{cond}\left(x_{d}\right)_{m}$ are convex polyhedra (i.e. described //by a finite number of linear inequalities)

$$
\text { \} }
$$

From steps of 5 and 6 in the above algorithm, we see that this algorithm has a linear time complexity $O\left(n^{*} m\right)$, where $n$ is the number of atomic components of the input RTADEVS model, and $m$ is the maximum number of states in a single atomic RTA-DEVS component.

By applying the previous algorithm, we obtain a TA model that executes every transition defined in the RTA-DEVS model under study. As we know, the RTA-DEVS behavior is completely defined by its transition functions, which defines all transitions in RTA-DEVS model. Thus, the resulting TA model executes the RTA-DEVS. The behavioural equivalence of the resulting TA model to the original RTA-DEVS model has been verified theoretically with the bisimulation relation as we showed in sections 4.3 .1 , 4.3.2, and 4.3.3. This also was verified with a practical example as introduced in section 4.8 . 
Table 2: Transformation of RTA-DEVS to Behaviourally Equivalent TA.

\begin{tabular}{|c|c|}
\hline RTA-DEVS & Equivalent TA \\
\hline $\begin{array}{l}\text { Internal Transition } \\
\operatorname{ta}\left(s_{l}\right)=\mathrm{C}, \delta_{i n t}\left(s_{l}, e\right)=s_{3}\end{array}$ & $\overbrace{x<c}^{\mathrm{x}=0}$ \\
\hline $\begin{array}{l}\operatorname{ta}\left(s_{4}\right)=\mathrm{C}, \\
\delta_{e x(}\left(s_{4}, a, e\right)=s_{5}, \quad 0 \leq e<3 \\
\delta_{e x t}\left(s_{4}, a, e\right)=s_{6}, \quad 3 \leq e<\operatorname{ta}\left(s_{4}\right)\end{array}$ & clock $x$ \\
\hline
\end{tabular}

\subsection{Resolving rational numbers in RTA-DEVS model}

Timed safety automata, as defined in section 2.2 .1 , only deals with bounded integer variables. This restriction is made to guarantee the finiteness of state-space, and hence 
termination of reachability algorithm [5]. This may seem a limitation when one is trying to verify RTA-DEVS models which have constant values of type Real, in their transition functions. However, this is not much limitation, as any group of real rational numbers can always be represented as integer numbers. This is done in TA model by changing the model time scale. For example, imagine we have the following values as constants defined in RTA-DEVS transition function to represent time in minutes $(1 / 2,3 / 5,3 / 8)$. To convert these to integer numbers on an equivalent TA model, we multiply each of them by the least-common-multiple of all the denominators. The resultant automaton would be equivalent modulo time scaling. This makes the TA having the values $(1 / 2 * 40,3 / 5 * 40$, $3 / 8 * 40)=(20,24,15)$ and its time scale is $1 / 40$ of a minute. For a network of TA, i.e. many TA models interacting together in a larger model, the scaling has to be done in all the models in the network. Therefore, all constants in a network of TA would be multiplied by the scaling factor. This should not affect the verification results as long as any requirement to be verified is expressed as a system query, in which its constants are scaled with the same factor as well.

\subsection{DEVS to RTA-DEVS}

One of the main obstacles in verifying classic deterministic DEVS models through transformation to TA is the possibility of DEVS models to have irrational constant values in their internal or external transition functions. When doing the previous transformation from DEVS model to TA, this would produce TA model with irrational constant values in its guards or state invariants. However, such TA model reachability analysis would be 


\subsection{DEVS to RTA-DEVS}

One of the main obstacles in verifying classic deterministic DEVS models through transformation to TA is the possibility of DEVS models to have irrational constant values in their internal or external transition functions. When doing the previous transformation from DEVS model to TA, this would produce TA model with irrational constant values in its guards or state invariants. However, such TA model reachability analysis would be undecidable as shown in [69]. This implies that for any DEVS model containing state lifetime of irrational values, it will not be possible to directly apply the transformation shown in table 2. In this case, the irrational values would need to be approximated to the nearest rational value according to the modeller's choice, based on the required precision for the equivalent RTA-DEVS model. In doing so, the transformation should take into account the following rules. These rules when applied, avoid building invalid RTADEVS or TA models containing time-action locks (that prevent the model execution progress), or loops where execution progresses infinitely without allowing time to advance [77].

Rule I: When approximating an irrational value of a state lifetime, and the internal transition output of this state is coupled with an external transition, the choice of the approximation value should be consistent for all constants using this irrational number. Formally, if the DEVS coupled model as shown in figure 22 is defined as the following:

$$
\begin{array}{ll}
\delta^{A}{ }_{i n t}\left(S_{i}, C_{i r r}\right)=S_{j}, \lambda^{A}\left(S_{i}\right)=a, t d^{A}\left(S_{i}\right)=C_{i r r} \\
\delta^{B}{ }_{e x t}\left(S_{k,} e, a\right)=\left(S_{i}, 0\right) & C_{i r r} \leq e<\infty
\end{array}
$$




$$
\delta_{e x t}^{B}\left(S_{k} e, a\right)=\left(S_{m}, 0\right) \quad 0 \leq e<C_{r}
$$

Where:

$C_{i r r}:$ is an irrational real number.

$C_{r}$ : is a rational real number.

$\delta^{A}{ }_{i n t}, \lambda^{A}, t d^{A}:$ Functions defined for component $\mathrm{A}$.

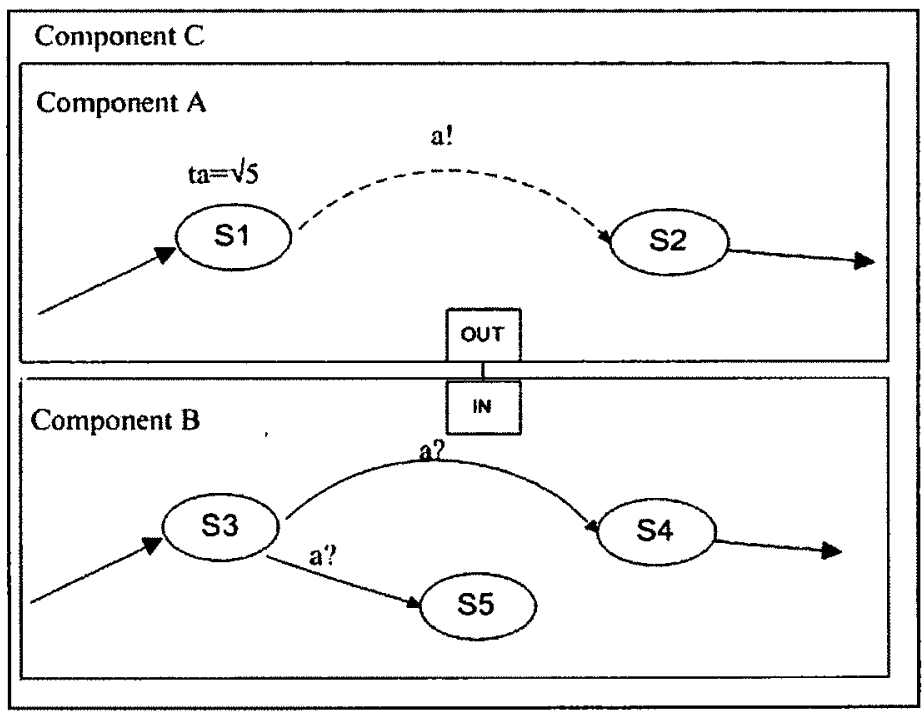

Figure 22: A coupled DEVS model.

Rule II: When approximating an irrational value for elapsed time in the definition of the external transition function, the choice of the approximation value should be consistent for all constants using this irrational number. Formally; if the following DEVS external transition function is defined in a model similar to the one shown in figure 23:

$$
\begin{array}{ll}
\delta_{e x t}\left(S_{i}, e, a\right)=\left(S_{j}, 0\right) & C_{i r r} \leq e<\infty \\
\delta_{e x t}\left(S_{i}, e, a\right)=\left(S_{k}, 0\right) & 0 \leq e<C_{i r r}
\end{array}
$$

It should be approximated in RTA-DEVS model on the following form to avoid creating action-locks: 


$$
\begin{array}{ll}
\delta_{e x t}\left(S_{i}, e, a\right)=\left(S_{j}, 0\right) & C_{r} \leq e<\infty \\
\delta_{e x t}\left(S_{i}, e, a\right)=\left(S_{k}, 0\right) & 0 \leq e<C_{r}
\end{array}
$$

The second rule is to avoid action locks that may happen if we define the external transition function with conditions on its transitions where there is a gap in time (in which the function is not defined). Another possibility is to have an approximated external transition function in which conditions on different transitions overlap in time, thus creating non-determinism that is not in the original DEVS model.

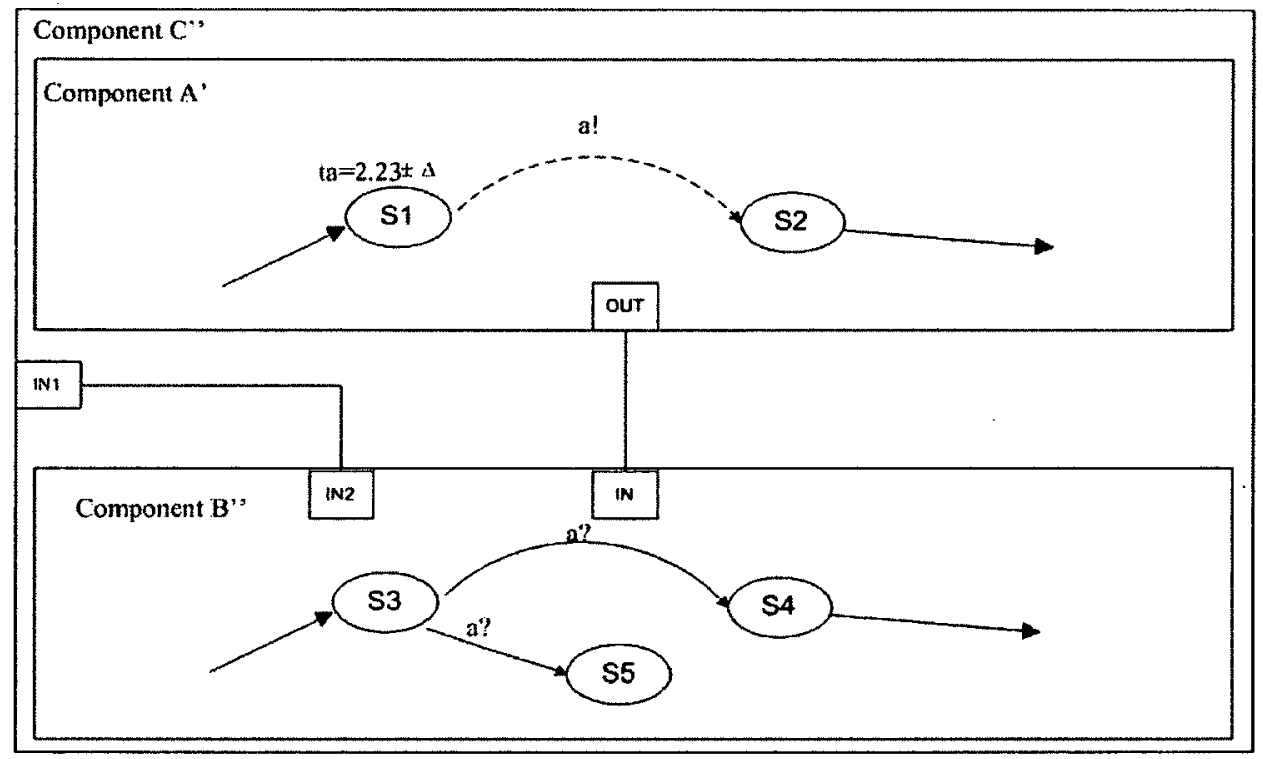

Figure 23: RTA-DEVS component with External Input.

\subsection{Estimation of Approximation to Verification Results}

When we approximate irrational values that may exist in a DEVS model to obtain the equivalent RTA-DEVS model, we get an approximation error $\Delta$ between irrational value and its approximated number. The next question is how the approximation of irrational 
constants in $t a$ or $\delta_{e x t}$ affect the formal verification of RTA-DEVS models. Would a result obtained from model checking RTA-DEVS models apply to the original DEVS?

When we approximate an irrational constant $C_{i r r}$ with a rational constant $C_{r}$, we introduce an error $\Delta$ such that $C_{i r r}=C_{r} \pm \Delta$. This error appears whenever $C_{i r r}$ is used in a time advance function or in an external transition function. Verification of RTA-DEVS through transforming it to an equivalent TA is done through reachability analysis. Would this analysis differ by introducing the error $\Delta$ when we move from DEVS to RTADEVS? Answering this question directly requires applying reachability analysis to the original DEVS with irrational constant values, and again for the transformed RTA-DEVS model with the rational values, then comparing results. This approach however is not feasible as reachability analysis for timed models with irrational constants is proven to be undecidable as shown in [69].

Therefore, there is a need to use an approximate approach to estimate the effect of $\Delta$ on the reachability analysis. This problem is equivalent to the problem of TA robustness [65]. A robust TA model accepts an input sequence of events within a time interval. Each event is accepted within a bounded time interval instead of an exact point in time as per normal TA. This is called a bundle of events that are close in time and the model still behaves the same with this bundle input.

Puri [78] extended the notion of robust TA to include models that their reachability analysis is unaffected with small drifts in clock models. In this definition, a model is not robust if for any small drift in clock rate, the reachability results change. In [66], De Wulf 
et al. proved that clock drifts in TA are equivalent to having a reaction delay by the implementation. This delay can be seen as an increase of guard constants by a small positive value $\Delta$. The robustness problem is then transformed to a problem in which one needs to find a value $\Delta$ that keeps verification results correct. Further work by De Wulf et al. [67] showed a methodology to assess a model for implement-ability by using standard TA model checking tools, and also showed a proof that if a model is tolerant to a certain value $\Delta$, it would also be correct with any value $\Delta^{\prime}$ such that $\Delta^{\prime}<\Delta$.

We used the results from the robustness theory of TA to check if formal verification results of a RTA-DEVS model correctly applies to the original DEVS model, and this work was published in [69]. Given an error $\Delta$ introduced by approximating irrational numbers in a DEVS models, we use an enlarged time interval with $\Delta$ to model the possible transition from a state in a non-deterministic fashion. For example if we have the following definition of DEVS external function $\delta_{\text {ext }}$ :

$$
\begin{array}{ll}
\delta_{e x t}\left(S_{i}, e, a\right)=\left(S_{j}, 0\right) & C_{i r r} \leq \mathrm{e}<\infty \\
\delta_{e x t}\left(S_{i}, e, a\right)=\left(S_{k}, 0\right) & 0 \leq \mathrm{e}<C_{i r r}
\end{array}
$$

and we have $C_{i r r}=C_{r} \pm \Delta$, then, we enlarge the interval in which the external transition is enabled, i.e. to define $\delta_{\text {ext }}$ as:

$$
\begin{array}{ll}
\delta_{e x t}\left(S_{i}, e, a\right)=\left(S_{j}, 0\right) & C_{i r r}-\Delta \leq e<\infty \\
\delta_{e x t}\left(S_{i}, e, a\right)=\left(S_{k}, 0\right) & 0 \leq \mathrm{e}<C_{i r r}+\Delta
\end{array}
$$

These modified intervals results in an overlap interval as shown in figure 24 . In this overlap, the TA behaves non-deterministically to choose either right or left time interval 
to follow. This non-determinism would cover all reachable states within reachability analysis, thus covering any value $\Delta^{\prime}$ such that $\Delta^{\prime}<\Delta$.
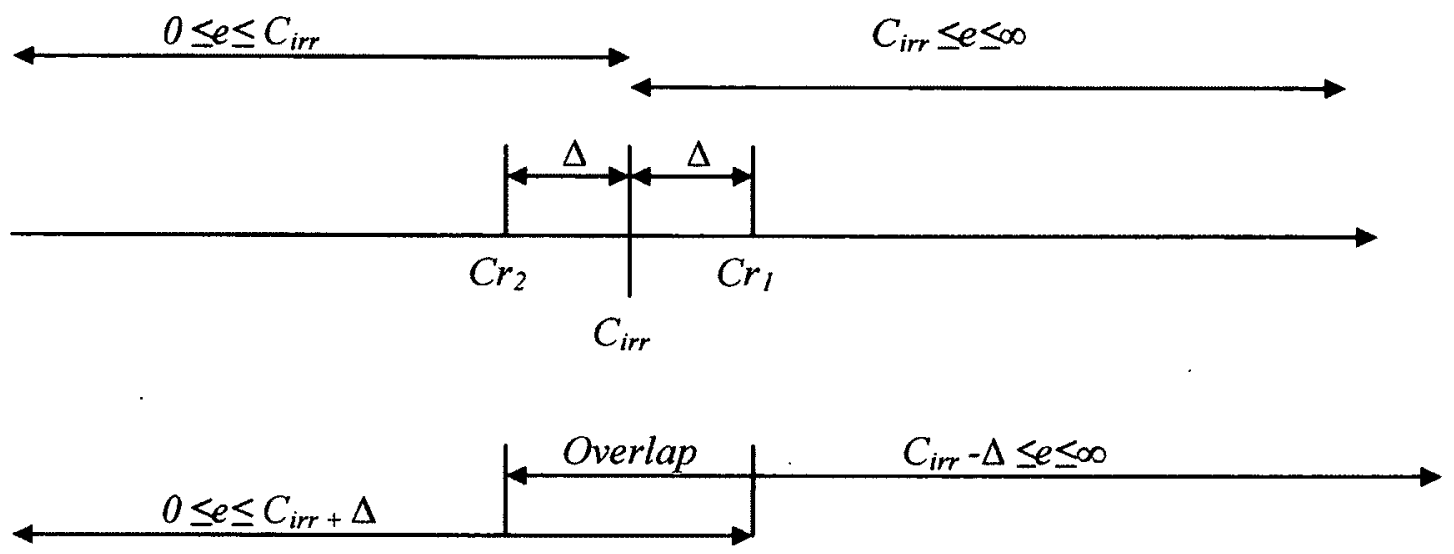

Figure 24: Overlap of two intervals because of approximating irrational value.

Using the above approach for irrational values, any DEVS model is transformed to an equivalent TA as shown in the previous section and as we have shown in [68]. This TA model is then checked against the desired properties. With non-determinism in the model, UPPAAL checks the transition function in the overlap interval, covering the point around the irrational number value. Hence, if the model checking results were correct, we conclude that the approximation did not introduce errors to the RTA-DEVS model. On the other hand, if the verification results show a violation to the desired properties, we can conclude that this model with the approximated value $C_{r}$ does not hold the desired properties. Hence, the designer needs either to select another value of $\mathrm{C}_{\mathrm{r}}$ by reducing the error $\Delta$, or if this is not feasible because the designer needs a model that can tolerate a 
large error value, the current DEVS model need to be revised to hold the desired properties and then it can be re-verified again to confirm this.

\subsection{Case study: an Elevator Control System}

To show the above-proposed methodology of estimating the effect of approximation on reachability results, we modified an example originally introduced in [72]. That example defines an elevator system composed of an Elevator, the Elevator Controller and an Environment that represents a user pressing different buttons. The elevator controller interacts with the user to receive button requests from each floor. Then, it makes the elevator move to respond to the user requests. This is an example of a (soft) Real-Time System with safety and bounded response time requirements. This example was transformed from RTA-DEVS to TA, and verified to work correctly in UPPAAL. A summary of this case study, originally presented in [68], is given below.

The elevator model shown in figure 25 represents the different states of the elevator movement and transitions between these states. This is an abstract model of the elevator where some details like door operation, floor display, etc. have been ignored (as we are only interested in controlling the elevator movement). The elevator starts in the stopped state and waits for the controller commands to move (satisfying a button request from the user). The controller takes the decisions for direction, start and stop. of the motors.

The elevator DEVS graph model in figure 25 has 5 external transitions, shown with solid arrows; and three internal transitions (one internal transition from aux state to stopping state is hidden under the external transition shown in the figure), shown with dotted 
arrows. Note that an external transition is enabled whenever the expression on that transition evaluates to true in RTA-DEVS model.

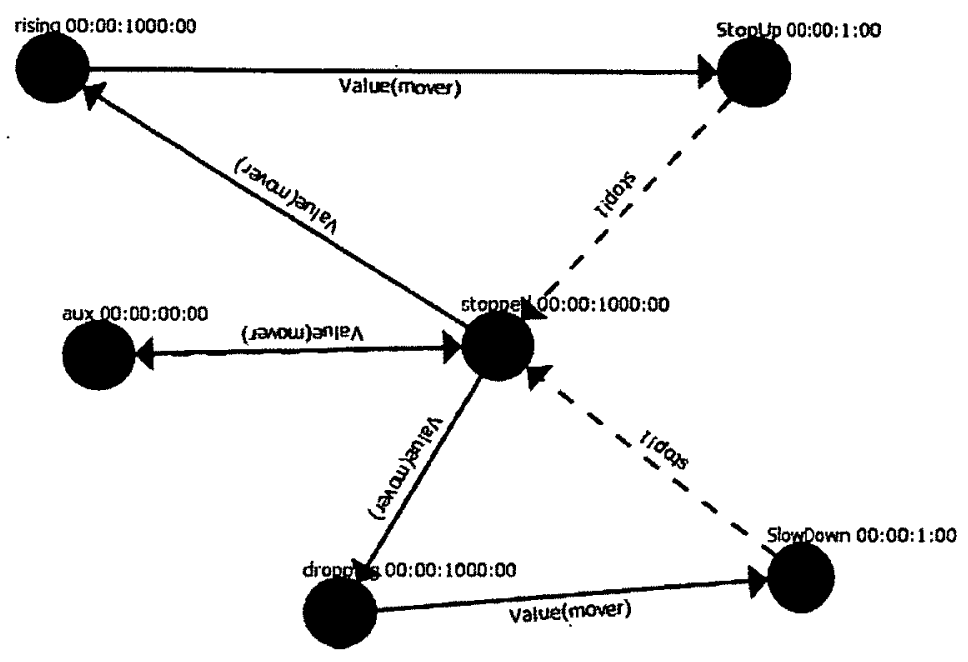

Figure 25: Elevator RTA-DEVS Model.

The expression Value(mover) evaluates to true whenever the elevator model receives a value in mover variable equals to 1 . This expression is translated to a channel reception move? as shown in the TA model in figure 26 . In this case, whenever a value is transmitted on that channel, the transition synchronized on that channel is enabled. By taking each transition from the RTA-DEVS model and applying the previous steps of the algorithm in section 4.4 , we get the behaviourally equivalent TA model shown in figure 26. Also note that time advance values for each state in RTA-DEVS model, has been substituted with an equivalent clock invariant in the corresponding state in the TA model, and the constant in that invariant equals state lifetime as indicated on the RTA-DEVS model. 


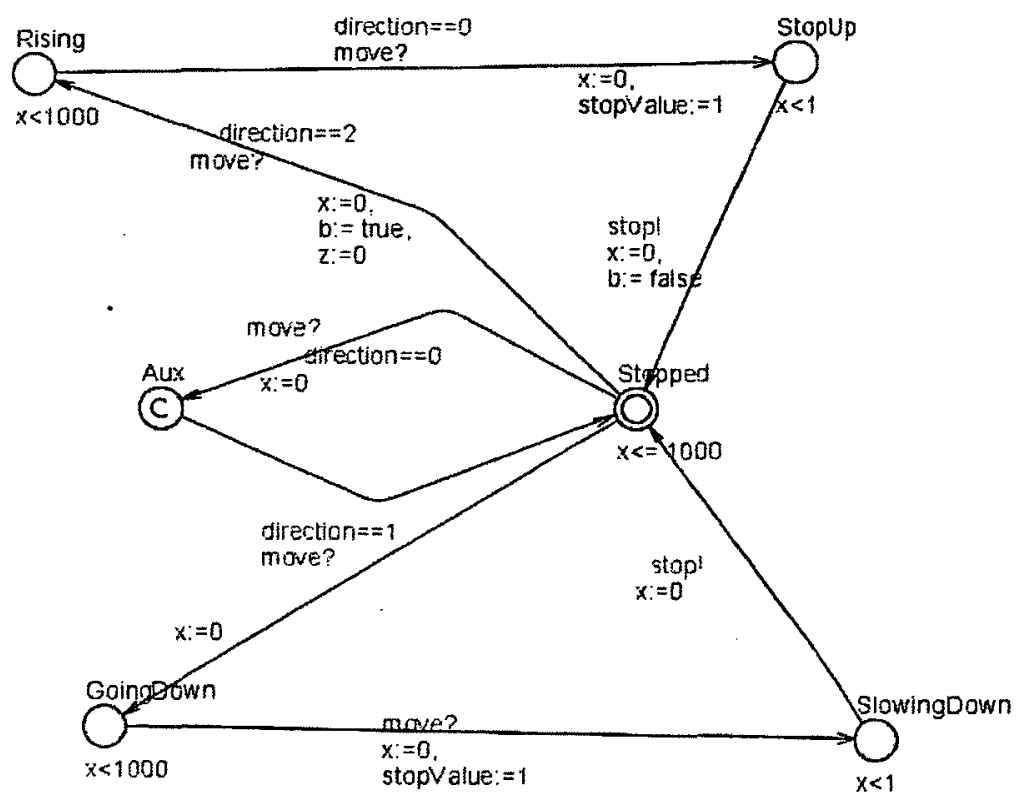

Figure 26: Elevator TA model.

The elevator controller is also responsible to interact with the user and send commands to the elevator to satisfy the user requests. The controller RTA-DEVS model is shown in figure 27 and represented in DEVS graphs notation. In this model, we abstract the behaviour of the controller to being in one of possible 6 states. These states represent the elevator in regards to its movement direction and its acceleration. The states are StdByStop that represents the elevator in a complete stop and ready to move for any coming requests, Moving in which the controller makes a decision to move the elevator based on current floor and the button pressed floor, StdByMov corresponds to the elevator moving to the desired floor and the controller in that state receiving sensor signals to decide when to stop the elevator, aux which serve as a dummy state with internal transition that is executed immediately after reaching that state, the state purpose is to 
enable the test of the sensor value on the external transition to it with the function equal(sensor,floor), Stopped which corresponds to the controller deciding to send a signal to the elevator to slow in preparation to stop, and Stopping corresponds to the controller waiting for the elevator to get into complete stop and send a stop signal to the controller.

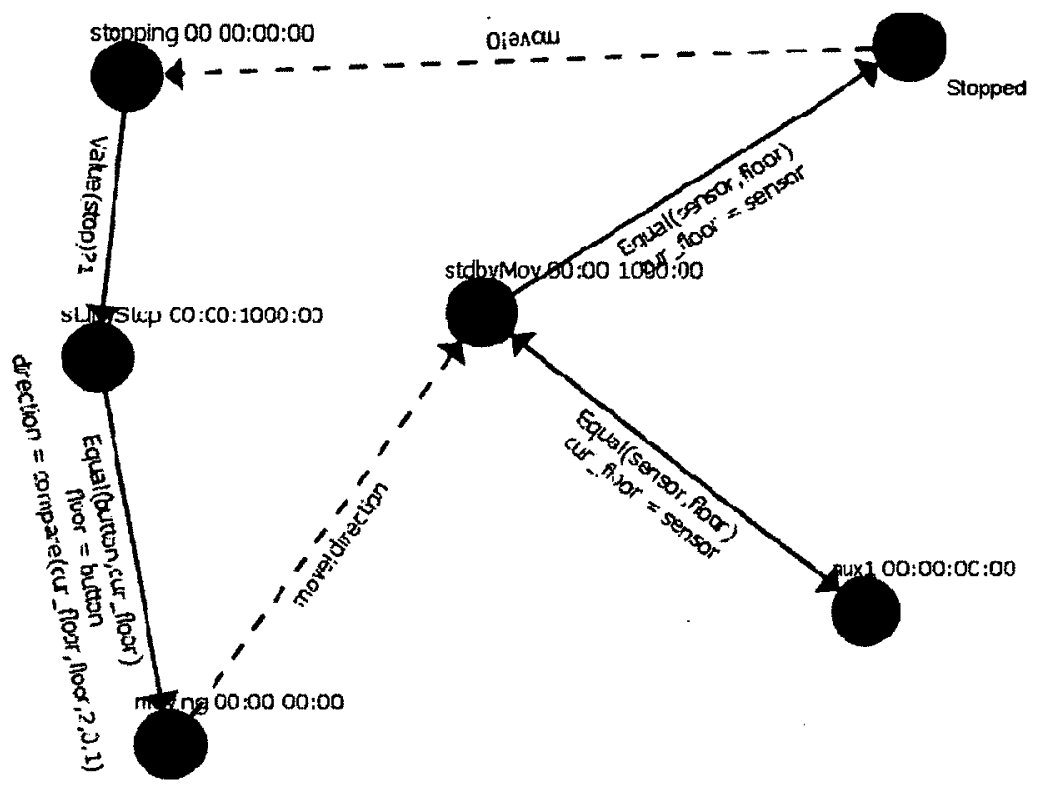

Figure 27: Elevator Controller Model as DEVS Graphs.

In this model, the controller would be in StdByStop state waiting for a button request to move. Whenever it receives the button request, it would trigger an external transition, and compare the button floor to the cur floor of the elevator. Based on this comparison, the controller would determine the direction in which the elevator should travel to, and stores this info into the direction variable. The controller then reaches Moving state that has a lifetime of zero time units. Therefore, an output function is executed to send the direction information through the port move to the elevator model, and an instantaneous (with no time advancing in Moving state) internal transition would be triggered to change the state 
into StdByMov state. The controller then decides to change to stopped state if the sensor reading matches the desired floor; otherwise, it would loop between aux state and StdByMov states as shown in the figure (please note the double head arrow between states StdByMov and aux that shows two transitions in opposite directions).

We applied the transformation steps shown in section 4.4 to the elevator controller RTA-DEVS model to obtain TA shown in figure 28.

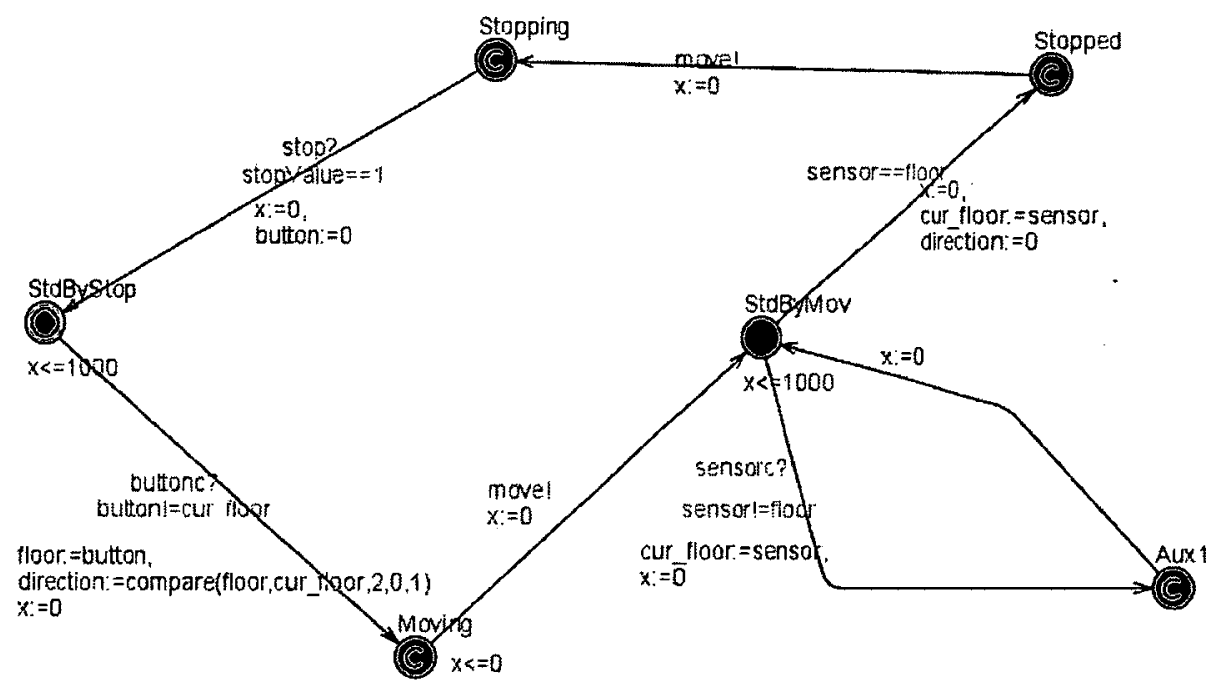

Figure 28: TA Controller model in UPPAAL.

In order to model input to the system, we construct an automaton that would send the button and sensor inputs to the controller as in figure 29. This automaton is necessary to make the TA system under study a closed model. In order for model checking techniques to be able to verify desired properties, they must work on closed systems as model checking would explore all possible system transitions to be able to determine if the desired property is met or not. Therefore, a good modeling of the system environment is also necessary to completely check all possible system behaviours for all expected 
environment inputs. However, modeling different environment behaviours is out of scope of this thesis and it is left for the modeller to choose a model that best describes system environment. In this thesis, we built a simple environment to test our verification methodology to DEVS model. In other systems, the modeller may need to build a sophisticated environment model that covers a bundle of scenarios as an input to the system being verified. TA models can cover a range of scenarios as it can be nondeterministic; however a suitable DEVS class with non-deterministic behaviour has to be chosen to model such an environment.

The environment modeled in figure 29 is responsible for sending button and sensor events to the controller. It starts at $S I$ state, after staying in this state for 5 time units, it sets variable button to 3 , then synchronizes with controller TA on channel buttonc. Again, waits in state $S 2$ until its clock $y$ reaches 10 time units, sets sensor to 1 , and synchronizes with the controller on channel sensorc. This process continues for the desired inputs sequences to the controller, and then resets the clock and restarts again at S1. 


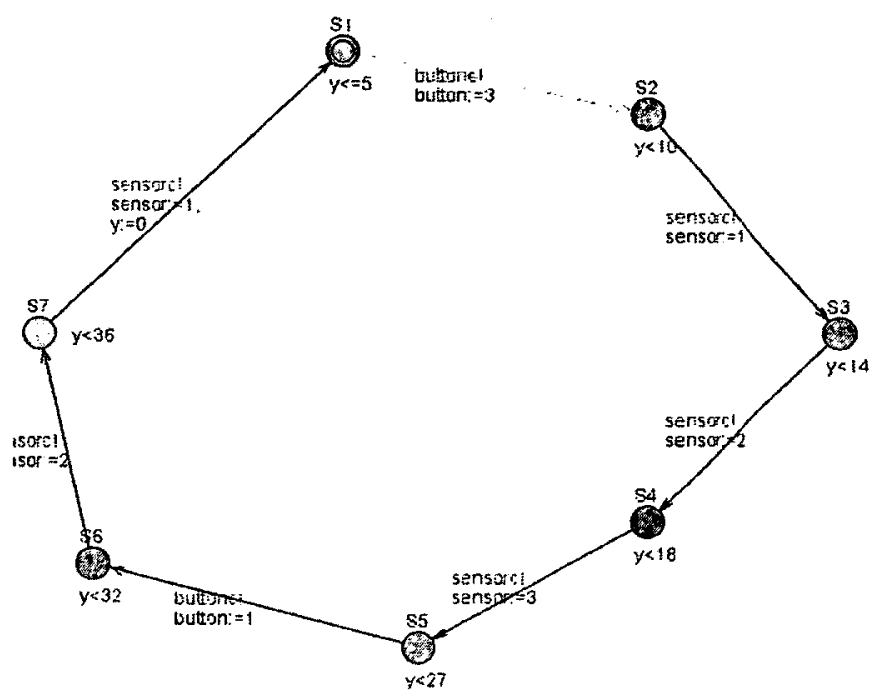

Figure 29: Environment inputs (Button and Sensor).

After translating our DEVS model to an equivalent TA model, we can use model checking to answer questions about our DEVS model behaviour that otherwise would have needed to fully simulate all possible executions of the DEVS model to get the answers. Some of the important questions would be:

a. Does our DEVS model execution stop at one point without being able to progress (having a deadlock)?

b. If no deadlocks are found in the DEVS model, is it always guaranteed whenever a user pushes a floor button that the elevator would reach that floor (normal operation as desired for the elevator system)?

c. In case the elevator eventually reaches the requested floor, is there a time upper bound between the request and the arrival of the elevator that our model would always guarantee to happen? 
In order to answer these questions, we formulated these questions into formal queries to the TA model. For the first question, we applied the UPPAAL verifier to our model to check for any deadlocks that may be present in the elevator model. To check for that failure, we have formulated a simple query in UPPAAL. It is expressed in UPPAAL Computation Tree Logic CTL query language [9] as:

\section{A[] not deadlock}

After running the checker, it shows that this property is satisfied, i.e. there is no deadlock in the DEVS model as results shown in figure 30.

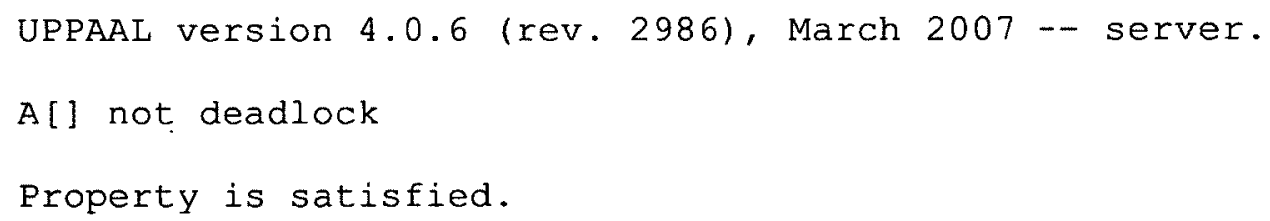

Figure 30: Elevator Verification Results in UPPAAL

To answer the second question, we need to check for the liveness property, i.e. something would eventually happen. In our case, for the proper operation of the controller within the coupled system, we are interested to check if by pressing a certain floor button, the elevator would eventually reach that floor. For example, if the user 
presses the $3^{\text {rd }}$ floor button, the elevator would eventually reach the 3 rd floor. This property is expressed in CTL as:

$$
\text { button }==3-->\text { ElevatorController.cur_floor }=3
$$

This property was satisfied as well in UPPAAL model checker for the given model. However, for a query as:

$$
\text { button }==3-->\text { ElevatorController.cur_floor }==4
$$

the property is not satisfied as we expect. By pressing $3^{\text {rd }}$ floor button, given the elevator initially stopped at $1^{\text {st }}$ floor, there is no way the elevator would reach the $4^{\text {th }}$ floor.

To answer the third question, i.e. to know if the elevator would reach the requested $3^{\text {rd }}$ floor within some bounded time. We extend the model for bounded time checking by adding Boolean variable $b$, and a global clock $z$ as shown on the Elevator model. Variable $b$ would be set to true as long the elevator starts traveling and until it reaches the Stopped state again. Therefore, by checking the accumulated time while $b$ is true, it would give us the property we need to check. Then, the property would be expressed with the following query:

$$
\text { A[] }(b \text { imply } z<27 \text { ) }
$$

which is satisfied. However, the following query is not satisfied:

$$
\text { A[] ( b imply } z<26 \text { ) }
$$

This shows that the elevator would reach the $3^{\text {rd }}$ floor after requested there by no less than 26 time units, but guaranteed to be there at 27 time units or more. More complex queries to check for more properties could also be formulated and verified by UPPAAL in case that we have a more complex DEVS model. 


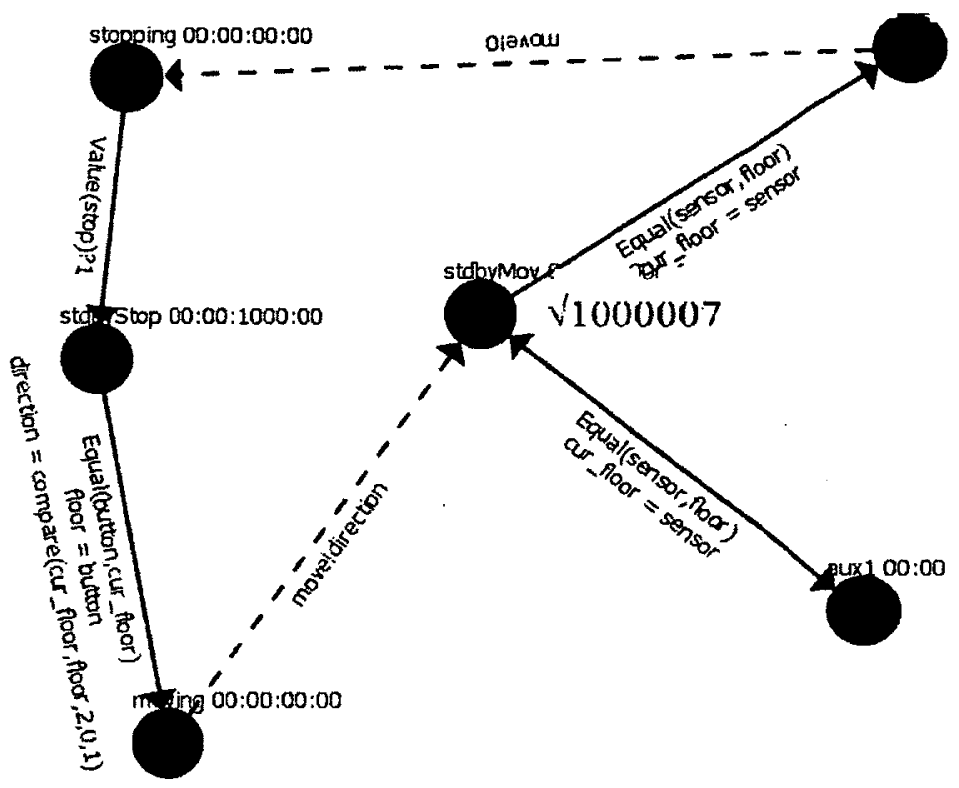

Figure 31: Elevator-Controller in DEVS Graphs notation with irrational value.

To show our proposed methodology for approximation error estimation, we changed the example by introducing an irrational value in the controller model. State stdbyMov in figure 31 would now have time advance value of $\sqrt{1000007}$ instead of 1000 as was in figure 27. This value can be approximated as $\sqrt{1000007} \approx 1000.003$ or $\sqrt{1000007} \approx$ 1000.004 , thus giving an approximation error $\Delta=0.001$.

These approximations are rational numbers and to obtain integer numbers on the equivalent TA model, we used the method discussed in section 4.5 to re-scale time on the model by multiplying the initial scale by 1000 . The resulting TA model is shown in figure 32. 


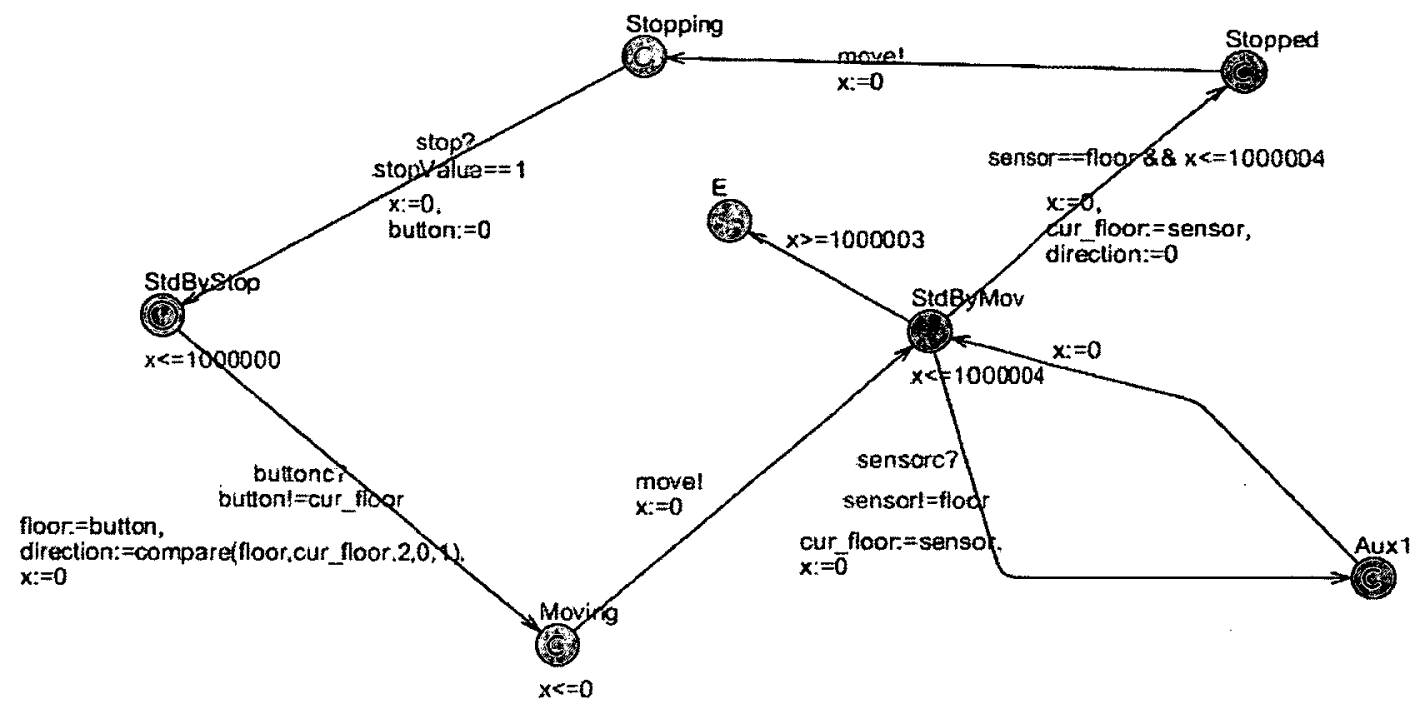

Figure 32: TA Controller model with Non-deterministic behaviour.

In this model, we added node $E$ and a transition from StdByMov to $E$ that is enabled at elapsed time of $x \geq 1000003$. This TA is semantically equivalent to the DEVS model in figure 31. However, this TA allows the transition from node StdByMov to node Stopped to be taken non-deterministically in the interval $[0,1000004]$ while transition to $E$ is enabled in $[1000003, \infty]$. This ensures covering the interval $[0, \sqrt{ } 1000007]$ in UPPAAL model checking.

The model checker was run to verify the non-deterministic version of the elevatorcontroller model along with the other components in the elevator system as we showed in [68]. The verification results were successful as was before for the deterministic version in figure 28. This indicates that the approximation error did not affect the verification results. Hence, for any value smaller than 0.001 , the results would not be affected as was proven by De Wulf in [67]. 
Although we could not verify the DEVS model in figure 31 directly as a result of the irrational value of time advance function, our methodology approximated this model to a behaviourally equivalent TA, which could be used to verify the TA model.

\subsection{Known Methodology Limits}

It is a well-known problem of all model checking techniques that the number of nodes in the reachability graph grows exponentially in the number of components and number of states of each component. This is called the state-space explosion problem. Model checking algorithms need to store a representation of the full reachability graph to be able to terminate after visiting all nodes of the graph. Due to the exponential growth of reachability graph, even if timed model checking is decidable with TA, there is a limitation of the practical size of models that can be checked with current tools and hardware.

This limitation also limits the size of DEVS models that can be verified with our methodology. However, as we show in section 6.1.1, our methodology can be extended by some techniques that reduce the size of reachability graph and hence scale the methodology to verify larger sizes of DEVS models. 


\section{Chapter 5: Hybrid DEVS Systems Verification.}

\subsection{Continuous-Discrete systems Verification}

In chapter 3 of this thesis, we introduced a methodology to enable the verification of deterministic and discrete DEVS models. Although the method is useful for deterministic discrete systems, in the case of RT embedded systems a discrete controller needs to interact with some physical environment that is continuous in nature. Examples of these are automobile cruise-control systems, boiler temperature or pressure control systems, heating and air conditioning systems, and power-plant systems. The controlled variables in these environments are continuous, and they are modeled using differential algebraic equations. However, the computer controller system is a discrete digital system. This makes the models of control systems a hybrid between continuous and discrete component models.

In order to overcome the problem shown in section 2.4 for hybrid systems verification, we introduced a technique within the DEVS formalism to model, simulate and verify hybrid systems. This technique uses the QSS method shown in section 2.4.1 to provide a way to model the continuous components. With this ability, we have an overall methodology in which a designer is able to model and simulate hybrid control and embedded systems within DEVS. Then, with this research results, the designer would be 
able to formally verify the hybrid model to guarantee the system correctness. This methodology is built on the verification of DEVS \& RTA-DEVS models discussed in chapter 3 and also on the use of the QSS method. As a DEVS model is executable on any embedded DEVS platform, for instance the CD++ engine (e-CD++) [8], the verified digital controller component can be deployed as an executable on the target system running e-CD++.

To accomplish the above goal of the thesis, we extend the previous work of verification of DEVS models done in chapter to include a special method for transforming continuous models expressed in QSS to TA. This enables the verification of hybrid DEVS models through model checking tools like UPPAAL.

To show the main ideas for hybrid systems verification within DEVS \& QSS, and potential problems and challenges that we need to solve, we present here a simple example of a simple continuous formula that is used for modeling many physical and natural systems. This is an exponential decay formula that describes an amount decreasing exponentially with regard to time. Some of the natural systems that follow this are [79]:

- Radioactive decay, where a radioactive material undergoes a nuclear change, the number of remaining atoms, from an initial amount, follows the exponential decay formula.

- The decrease of a temperature of a hot object cooling in a natural convective mode, such as a space-heating radiator. 
- Atmospheric pressure, which decreases as the latitude increases above sea level.

Some of these examples could be a part of a hybrid system composed of the physical environment and a digital controller, such as a thermostat-controlled heating of a room space. The temperature of the room radiator decreases exponentially with respect to time while the thermostat is in the off state. The exponential decay formula is modeled as follows, using an ODE:

$$
d x / d t=-x(t)
$$

Which has the analytical solution $x(t)=e^{-t}$, with the initial condition $x(0)=1$

Figure 33 shows a graph of the exact analytical solution of the exponential decay formula $x(t)=10 e^{-t}$ where $x(0)=10$.

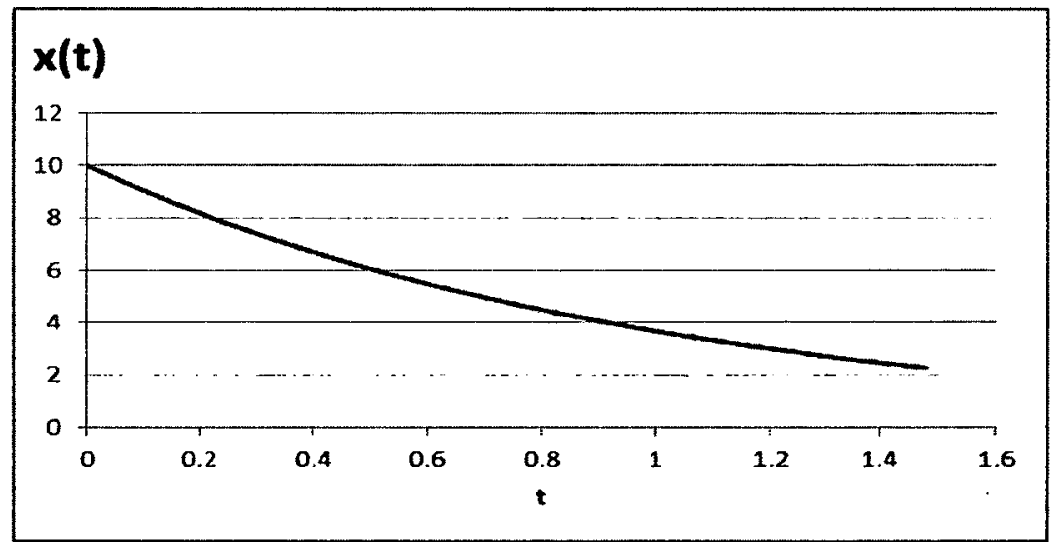

Figure 33: Exact solution for the exponential decay formula.

This simple ODE has an exact analytical solution, which is not the case for many other complex differential equations that exist in real-world applications. In those cases, the traditional technique is to use numerical methods to obtain an approximated solution. 
This approximated solution can be made arbitrarily accurate with a small error difference from the expected exact solution. In most applications, this inaccuracy is acceptable as the measurements of any physical value are of limited precision. Further, the modelling process involves abstracting many irrelevant details from the real system, which adds to the inaccuracy of the final solution. For these inaccuracies, an approximated solution is acceptable as long as its error stays within the margin of these tolerated inaccuracies.

Numerical methods for solvịng Differential Algebraic Equations DAE that describe a physical system can be represented in discrete event form using the QSS method. This happens to be a convenient form to model complex systems with the hierarchical nature of DEVS. It also allows the integration of the QSS components with other DEVS discrete event components to model any hybrid system.

Model checking also needs a discrete finite representation of the system under verification, thus, QSS \& DEVS give the right system model for model checking provided we solve some of the problems we face when we apply model checking on QSS components. Identification and solving these problems is the focus of this section of the thesis. To illustrate the issues with verifying DEVS models done with QSS, we would use the example of the decay formula shown above. The solution of the decay formula of (Eq. 5.1) is approximated with linear segments in QSS as shown in figure 45 on page 130. Each linear segment starts from a quantization level $Q_{k}$, and ends at the next quantization level $Q_{k-1}$. The slope of a linear segment is calculated as per the system ODE, for (Eq. 5.1) $\quad d q / d t=-q$. From this slope, QSS calculates the time needed for 
the quantized variable $q$ to move from $\mathrm{Q}_{\mathrm{k}}$ to $\mathrm{Q}_{\mathrm{k}-1}$ as $\sigma=d q /$ slope $=d q / q$. These calculations are part of the definition of the internal transition function $\delta_{i n t}(s)$ in (Eq. 5.2). The system starts with an initial state $x(0)=1$ which is modeled with the quantized state $q=\mathrm{Q}_{0}=1$. The quantum $d q$ chosen in this example is 0.1 , thus $\mathrm{Q}_{\mathrm{k}}-\mathrm{Q}_{\mathrm{k}-1}=0.1$, i.e. $q_{k-1}=$ $q_{k}-0.1$, because the function slope in this example is negative. This solution of (Eq. 5.1) is defined in a discrete-event form by the following QSS DEVS model:

$$
\mathrm{AM}_{\mathrm{D}}=\left\langle X, Y, S, \delta_{i n t}, \delta_{e x t}, \lambda, t a>\right.
$$

$X=\varnothing$

$S=\{s \mid s=(q, \sigma)\}$

$\operatorname{ta}(s)=\operatorname{ta}(q, \sigma)=\sigma$

$\delta_{\text {int }}(s)=\delta_{\text {int }}(q, \sigma)=(q-0.1,0.1 / q)$

$\lambda(q, \sigma)=q$

$q$ : is a quantized variable related to $x(t)$ system variable by a quantization function as shown in section 2.4.1. Figure 33 shows the exact analytical solution of the exponential decay formula $x(t)=10 e^{-t}$, which has $x(0)=10$.

Figure 34 shows the quantized representation of the decay formula as a result of simulating this QSS model, with $x(0)=10$. 


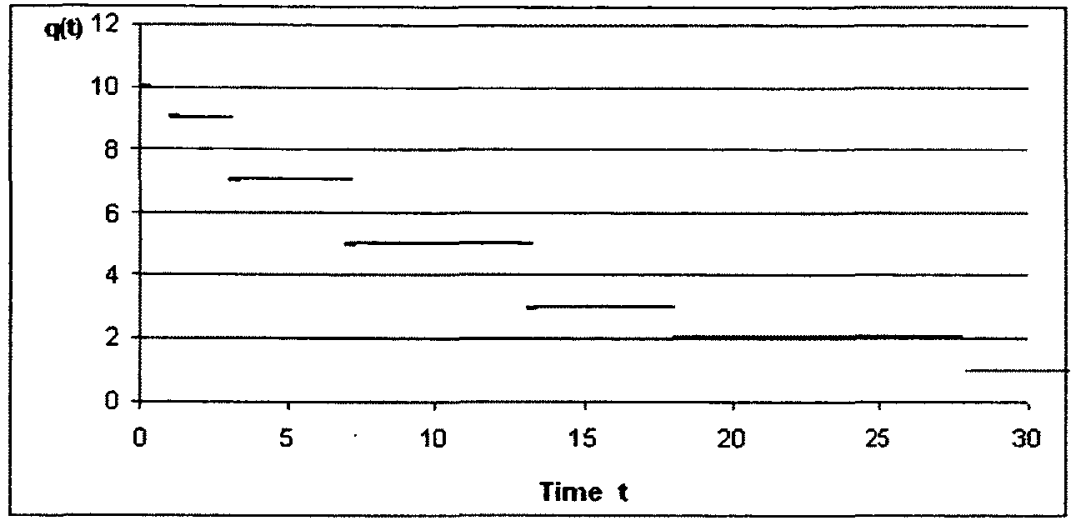

Figure 34: Quantized representation of exponential decay.

In the following section, we will look into the issues that we need to solve to enable hybrid DEVS verification. Please note that we do not try to propose an alternative method of solving continuous systems here. We only try to find suitable discrete representation that can be formally verified with the controller design. This discrete representation is obtained here using the QSS method.

\subsubsection{Transforming QSS DEVS models to TA}

To verify a general QSS system as defined in section 2.4.1 using timed automata, it would be necessary to represent it as an equivalent timed automata model while we preserve all the important properties that we may wish to verify, such as safety, reachability, and bounded liveness. To do so, we use an over-approximation model to contain all system behaviour defined in the QSS model. In order to do this, we need to solve the following:

1) The QSS model uses variables of type Real, which theoretically imply an infinite state space, while only Integer variables are supported in TA. This is to guarantee the 
finiteness of reachability state space, and hence the termination of TA reachability algorithm. Thus, we need an abstraction mechanism to reduce the infinite state-space of QSS variables to the bounded finite state-space of UPPAAL. However, this abstraction should preserve the critical properties in the hybrid system that we need to verify.

2) The exact behavioural equivalency between a continuous system modeled in QSS and TA cannot be done with bisimulation, as the QSS state variables are Real numbers, while TA have only Integer variables to represent the model states. We need to find a simulation relation between the continuous system and the QSS/TA model that preserves the important safety requirements.

3) A change of a variable representing a continuous system state is often described with arithmetic functions that return a Real number, and whose domain is also a Real number. These functions would need to be represented with approximated Integer arithmetic functions (for example, trigonometric, logarithmic or exponential functions).

To solve the first challenge, we need to represent the infinite continuous state-space with a reduced finite state space. This is easy as practical considerations put a limit to the number of states a QSS system would have for its variables. For example, it is common to use some variable $x$ to represent the value of a physical measure in a system. From the modelling process, it is possible to identify lower and upper bounds on the values of this variable in a given model. Further, any measured physical property would have a finite 
precision according to the measurement method. This limited precision would lead to a measurement error $|e r r| \leq x_{i+1}-x_{i}$, i.e. the least possible observed difference between two successive values of variable $x$, is equal or greater than the measurement error.

Therefore, these observations provide a finite state system where the bounds would constitute lower and upper saturation bounds $\mathrm{Q}_{0}$ and $\mathrm{Q}_{\mathrm{r}}$, and the quantum $\geq \mid$ err $\mid$.

Thus, we obtain the finite set $Q$ of the quantization levels defined for a QSS model:

$Q=\left\{Q_{0}, Q_{l}, \ldots, Q_{r}\right\}$ a set of real numbers where $Q_{k-1}<Q_{k}$ with $1 \leq k \leq r$,

$Q_{k}-Q_{k-1} \geq|e r r|$. In this case, the number of states of a QSS system would be at most $r+1$ states.

In order to meet the second requirement, we do not need total equivalence of behaviour between QSS model and TA model. To verify the QSS model behaviour, we need to include this behaviour within TA model, i.e. one way simulation relation (the TA model simulates the QSS model). This is obtained with an approximation that includes all QSS model behaviour and thus preserves the properties we wish to verify. This can be done with an over-approximation of the QSS model that contains all behaviours of the original QSS model plus some extra behaviour due to the over-approximation. One way to do this is to abstract each Real number with an integer interval, and this can be done to any degree of precision required. This produces an over-approximation that guarantees full inclusion of the QSS model behaviour. This, in turn, guarantees any reachability verification results on the over-approximation are true for the original QSS model. 
An over-approximation preserves safety and bounded liveness properties as Berard et al. have shown in [80]. These properties are very important when verifying hybrid systems. The safety property says that there is (are) no unsafe (or bad) state(s) reachable from the system initial state(s). The bounded liveness property says that, whenever an event occurs, the system would always respond within a specific time bound $t$.

An example of how safety and bounded liveness properties are preserved, by overapproximating the original model, is given in [80]. As these properties are the most important in an embedded control system, we claim that over-approximation would suffice for our purpose, and the main purpose of this stage of the research will be to verify such a claim.

To illustrate the over-approximation, figure 35 shows an example of overapproximation of Real numbers with an Integer interval. In this figure, the "small" bubbles (states) represent the Real values of a system state variable that changes during system evolution. To approximate the system behaviour, all states where the state variable in the closed interval $x=[1.0,2.0]$, could be represented by a single state, $S$, in which, the state variable value is over-approximated to this single state representing the values $[1,2]$. The state $S$ thus contains all possible system dynamics for variable $x$ in the interval $[1.0,2.0]$. Assume that the state where $x=1.4$ is a "bad" state, and we wish to check the safety of our system, expressed as a condition to never reach this state from the initial system state. Then, if the reachability analysis of the over-approximation shows that the state $S$ is not reachable from any initial system state, then we can guarantee that 
the bad state (which is contained in S) is also non-reachable in the original system, hence we prove our system safety. However, as indicated in [80], if the reachability analysis shows that we can reach the large state $S$, then we cannot conclude the safety of our system unless we refine the approximation to a smaller approximation and re-do the reachability analysis. This example shows how the safety property is preserved in an over-approximated system, i.e. whenever we verify an over-approximation for a safety property and the system proves to be safe, we conclude that the original system is also safe as explained in [80]. The same can be deduced about the bounded liveness property.

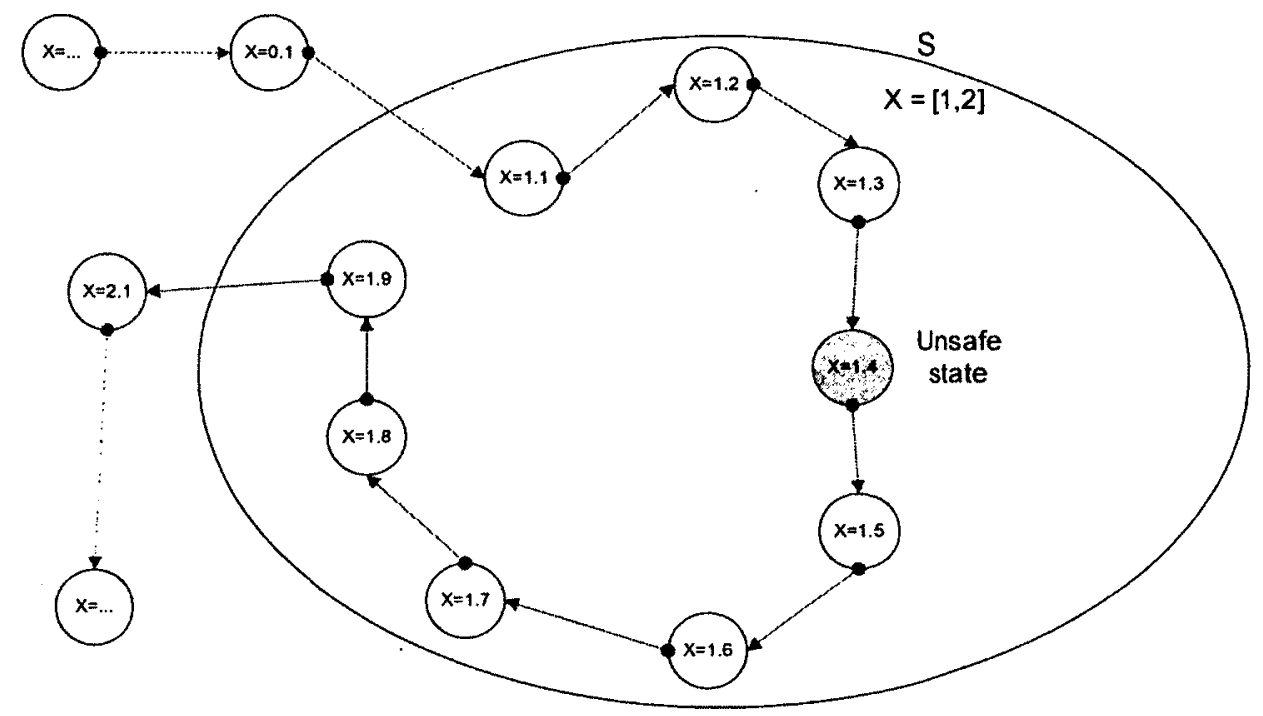

Figure 35: Example of real numbers over-approximation with integer numbers.

Now, we look into getting an equivalent TA model for the QSS model of the decay formula. To do so, we simulate the QSS behaviour with an equivalent TA model which 
contains this behaviour. First, we look at the semantics of the QSS model of (Eq. 5.2) which represents a loop as follows:

1. Initial values are assigned to $q=I$ and $\sigma=0$, resulting in initial state $S_{o}=(1,0)$.

2. After a time elapse of $e=\sigma$ the output function is triggered to send the value of $q$ to the outport, and then $\delta_{\text {int }}$ is triggered to calculate the next state $s$, which is composed of the new values of $q$ and $\sigma: s=(q-0.1,0.1 / q)$.

3. Repeat step 2 in the loop until $s=(0,10)$.

To transform this DEVS model to a TA, we need to solve the following issues:

1. The TA variables can only be of bounded integer type, in order to guarantee the finiteness of state space and hence the termination of the reachability algorithm. However, in QSS, state variables are real numbers and thus have infinite values.

2. The time of next quantum event $(\sigma)$ is approximated to an integer number. However, in doing so we need to preserve the original behaviour of the QSS model, and hence preserve the properties we need to formally verify.

The first issue is handled by converting rational Real numbers to Integers by multiplying all the values by the least common multiple of all the denominators. For any irrational values, we can use the technique we introduced in section 4.5 . For the second issue, we use abstraction by over-approximation [39]. With this technique, we approximate the real value of the event time $t_{i}$ with a bounded time interval such that $t_{i}$ $\in\left[T_{L}, T_{H}\right]$, where $T_{L}=$ floor $\left(t_{i}\right), T_{H}=$ ceiling $\left(t_{i}\right)$. This guarantees that the resulting TA would include all possible event timings in that interval. 
To obtain a TA that contains all the behaviour of the QSS model, we need a simulation relation with the QSS model (i.e., TA simulates QSS). To do so, each state in QSS would be simulated by a corresponding state in TA and each target state in QSS is simulated by a corresponding target state in TA. This is done in the TA model shown in figure 36 . Inspecting the semantics of this TA model, we can see the following simulation of the QSS model after multiplication by a scale of 10 to remove fractional parts:

1. The TA starts in the initial state $\mathrm{S} 1$, and moves to $\mathrm{S} 2$. On this initial transition, the total state variables are initialized as sigmaL $=0$, sigmaH $=0$, clock $\mathrm{t}=0$, and $q=10$.

2. After the time elapse $t$ where $\lfloor\sigma\rfloor \leq t \leq\lceil\sigma\rceil$ the transition $\mathrm{S} 2 \rightarrow \mathrm{S} 3$ is executed, calculating new value of $q=q-1$.

3. $\mathrm{S} 3$ is a committed state, causing the transition $\mathrm{S} 3 \rightarrow \mathrm{S} 2$ to be taken immediately, calculating new values, on this transition, for sigmaL $=\lfloor\sigma\rfloor$ and sigmaH $=\lceil\sigma\rceil$. The total state at $\mathrm{S} 2$ is $(q-1,\lfloor\sigma\rfloor \leq t \leq\lceil\sigma\rceil)$.

4. Steps 2 and 3 are repeated until the total state $=(q=1$, sigmaL $=\operatorname{sigmaH}=10)$.

To compare between QSS and TA semantics, we look at their states and transitions. From the above semantics of the QSS model, we notice that QSS has the following states and transitions:

$\left(q=q_{0,} t<\sigma_{1}\right) \stackrel{t=\sigma_{1} ; t:=0}{\longrightarrow}\left(q=q_{1,} t<\sigma_{2}\right) \stackrel{t=\sigma_{2} ; t:=0}{\longrightarrow}\left(q=q_{2,} t<\sigma_{3}\right)$ $\stackrel{t=\sigma_{3} ; t:=0}{\longrightarrow} \ldots . . \stackrel{t=\sigma_{10} ; t:=0}{\longrightarrow}\left(q=q_{10, t}<\infty\right)$ 
From the semantics of TA model of figure 36 , and after changing the model scale to eliminate fractions, this TA has the following states and transitions:

$\left(q=q_{0, t} t<\left\lceil\sigma_{1}\right\rceil\right) \stackrel{\left\lfloor\sigma_{1}\right\rfloor \leq t \leq\left\lceil\sigma_{1}\right\rceil ; t:=0}{\longrightarrow}\left(q=q_{1, t}<\left\lceil\sigma_{2}\right\rceil\right) \stackrel{\left\lfloor\sigma_{2}\right\rfloor \leq t \leq\left\lceil\sigma_{2}\right\rceil t:=0}{\longrightarrow}\left(q=q_{2}\right.$, $\left.t<\left\lceil\sigma_{3}\right\rceil\right) \stackrel{\left\lfloor\sigma_{3}\right\rfloor \leq t \leq\left\lceil\sigma_{3}\right] t:=0}{\longrightarrow} \ldots . .\left\lfloor\stackrel{\left\lfloor\sigma_{10}\right\rfloor \leq t \leq\left\lceil\sigma_{10}\right\rceil t:=0}{\longrightarrow}\left(q=q_{10}, t<\infty\right)\right.$

From this, we can see that, for each transition in the QSS model, every state in the QSS model is simulated by a state in the TA model. It results that the TA of figure 36 simulates the above QSS model, and is an over-approximation as it contains all the transitions and the states of the QSS model.

In implementing this TA model, the calculated value of the next event $\sigma$ is overapproximated within an integer interval $[\lfloor\sigma\rfloor,\lceil\sigma\rceil]$ with the following functions we defined in the UPPAAL model:

$\operatorname{round} U p \operatorname{Div}(x, y)=\lceil x / y\rceil$

roundDownDiv $(x, y)=\lfloor x / y\rfloor$

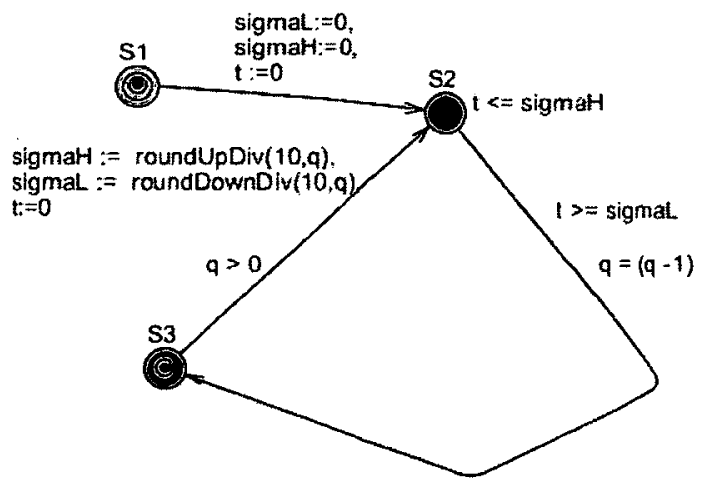

Figure 36: TA model of exponential decay formula. 
In this timed automaton, the state variable is represented with an integer variable $q$. This TA model produces the quantized output through the values of $q$ that represent the state variable. The output of this model over-approximates what is seen in figure 34 .

\subsubsection{Case Study of a Hybrid Elevator System}

To show how this integrates into a methodology for verification of hybrid DEVS models, we modified the example originally introduced in section 4.8 to contain a continuous model describing the dynamics of elevator braking. We also published a summary of this case study in [81].

To extend the Elevator case study we introduced in section 4.8 , we consider a continuous model of the elevator de-acceleration motion due to applying the brakes. This model can be described by a differential equation as:

$$
\frac{d v}{d t}=a
$$

Where $v$ is the elevator speed, and $a$ is a constant acceleration which is a negative value in case of braking or a positive value for moving out of rest. Figure 37 shows a negative constant acceleration representing braking action applied on the elevator. 


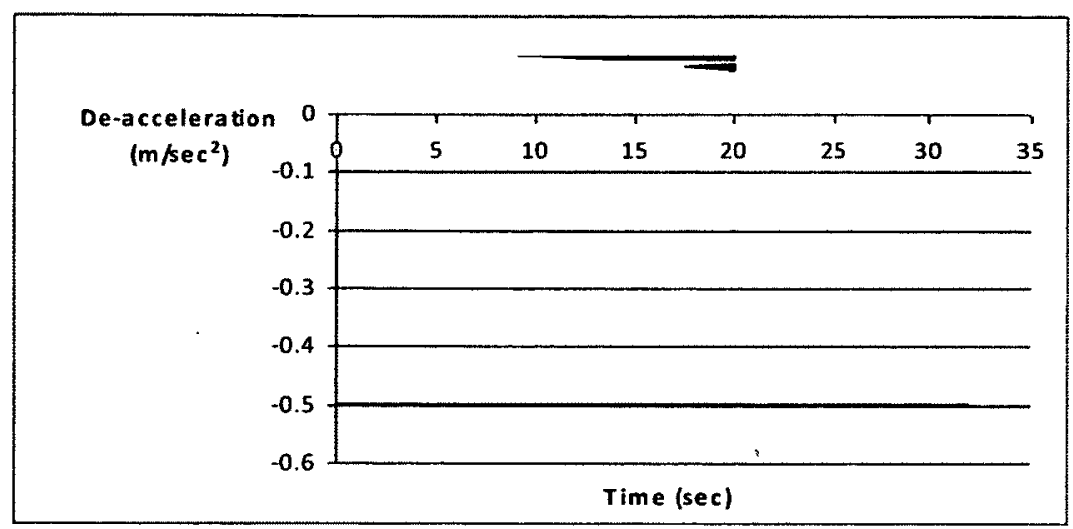

Figure 37: Elevator braking. De-acceleration: $0.5 \mathrm{~m} / \mathrm{s}^{2}$.

The speed of the elevator at any point in time $t$ can then be obtained as:

$$
v=a t+v_{i}
$$

With $v_{i}$ the initial elevator speed before applying the brakes. figure 38 shows the change in elevator speed during braking. The elevator brakes are applied while its speed is $4 \mathrm{~m} / \mathrm{s}$, and the subsequent reduction of speed is shown until complete stop.

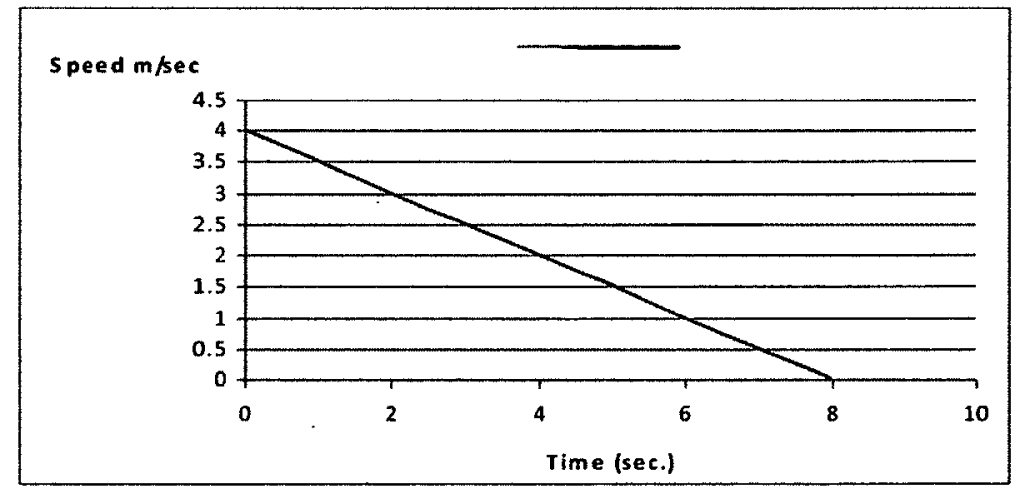

Figure 38: Elevator speed under braking.

With this continuous model of the elevator motion, our overall elevator model becomes a hybrid between discrete and continuous components. To simulate and then verify this 
hybrid model, we must obtain discrete representation of the elevator braking model. This can be done within DEVS formalism using the QSS method. A QSS model to represent the elevator speed under braking (Eq.5.4) can be described as follows:

$\mathrm{AM}_{\mathrm{D}}=\left\langle X, Y, S, \delta_{\text {int }}, \delta_{\text {ext }}, \lambda, t a\right\rangle$

$X=\varnothing$;

$S=\{s \mid s=(q, \sigma)\}$

$\operatorname{ta}(s)=\operatorname{ta}(q, \sigma)=\sigma ; \quad \delta_{\text {int }}(s)=\delta_{\text {int }}(q, \sigma)=(q-0.5,0.5 / a)$

$\lambda(q, \sigma)=q$

$q$ : is a quantized variable related to $v(t)$ system variable by quantization function as shown in section 2.4.1. Figure 39 shows the quantized output of this QSS model. $a$ : is the slope value as defined by (Eq.5.3).

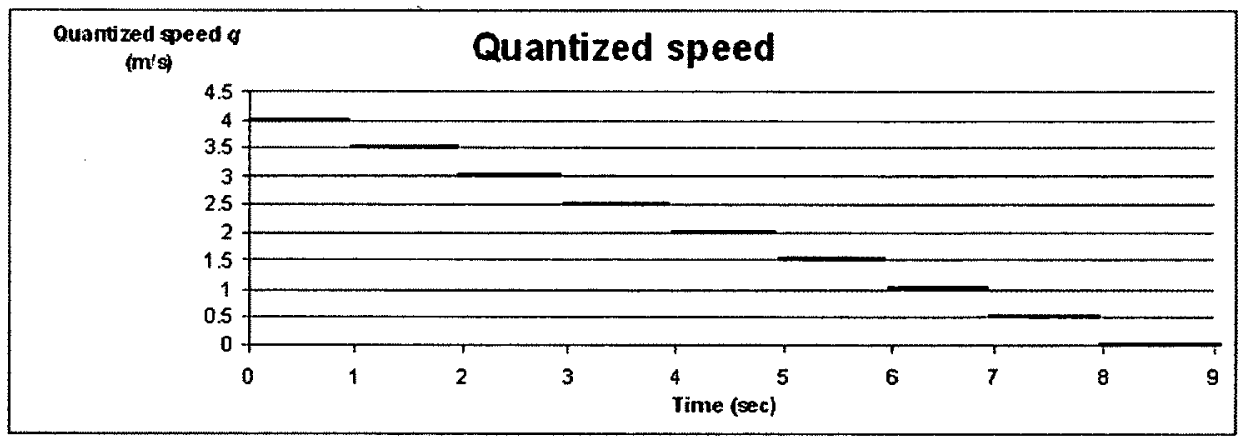

Figure 39: Quantized braking-elevator speed. 
To enable the formal verification within UPPAAL to the combined hybrid elevator model, we transform the QSS model of (Eq.5.5) to an equivalent TA model as per the method shown above, resulting in the TA shown in figure 40 .

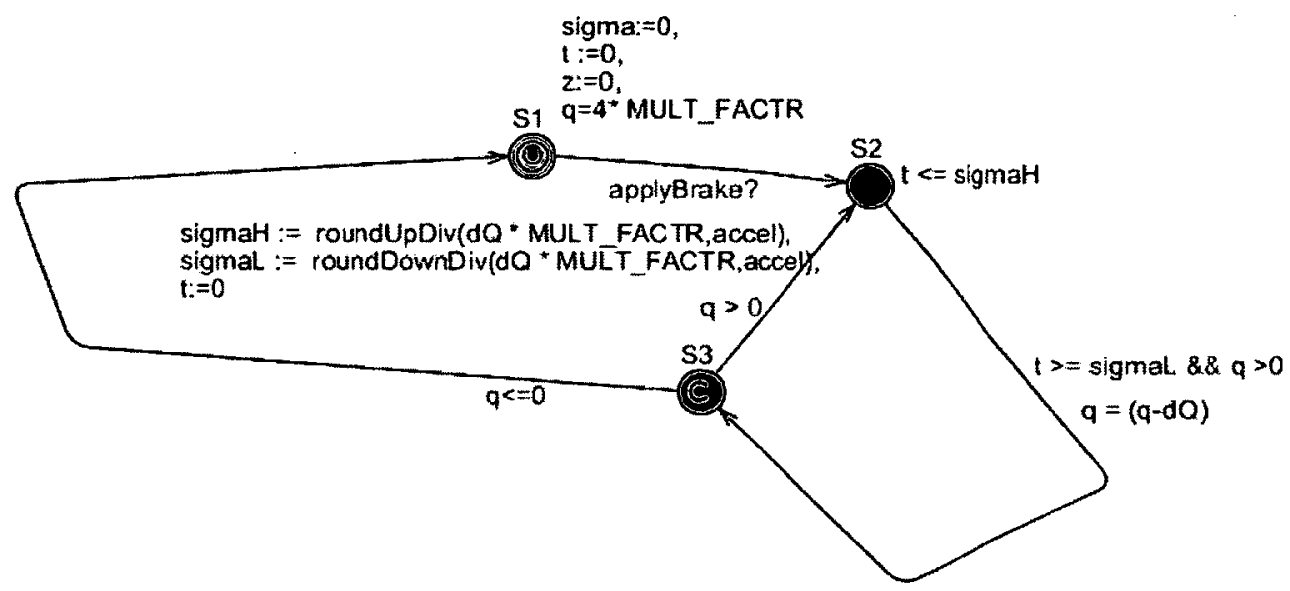

Figure 40: TA model of braking elevator motion.

Sigma is calculated as per the definition of (Eq.5.5) within the $\delta_{\text {int }}$ function. However, sigma is defined as a real value in the QSS model. Therefore, sigma value is overapproximated with an integer interval $\sigma \in[$ sigmaL, sigmaH]. Thus, the TA model behavior includes all the trajectories $(q, t)$ where $q$ is the quantized state, and $t \in[$ sigmaL, sigmaH]. In addition, since TA deals with only integer type variables, we multiplied all values of the QSS model by a factor of 100 to convert all fractions to integers. This multiplication is done on all TA components in the elevator model to scale the time evenly for all the component models. We note here that this multiplication, though necessary to convert rational constants to integers, yields the total reachability graph size larger as number of nodes in reachability graph increases with the maximum constant 
value in a TA model. This is noted in section 6.1 .1 for future enhancements to this methodology. Another modification to the original elevator model was necessary to enable the elevator to communicate with the QSS model through the synchronization channel applyBrake, and for the elevator to move to the Stopped location only when the quantized speed value $q$ reaches zero. The modified elevator model is shown in figure 41 .

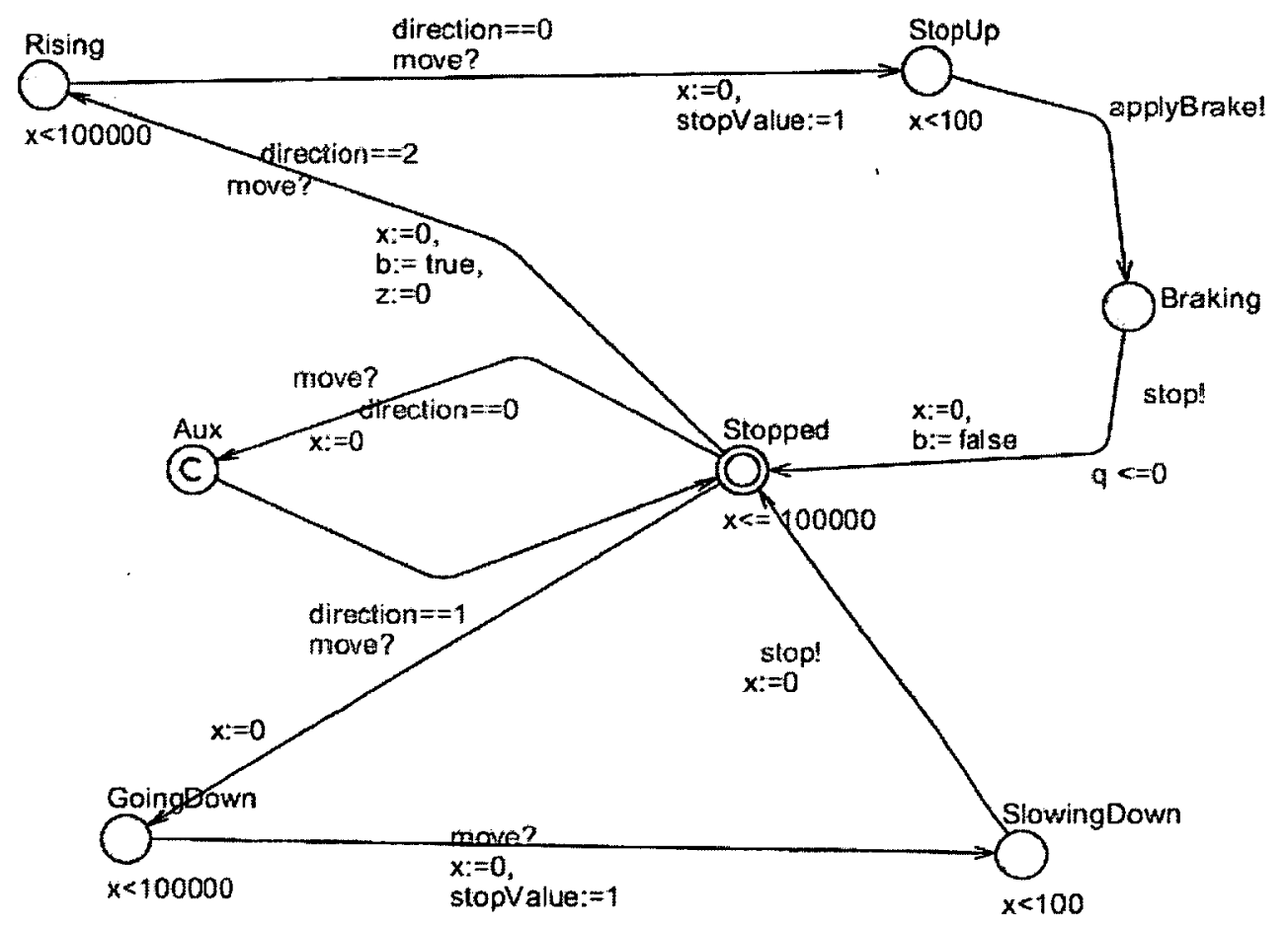

Figure 41: Modified elevator TA model- scale of $1 / 100$ second..

In this model, whenever the elevator receives the command from the controller to stop, it synchronizes with the braking motion model with sending applyBrake!. This would start the computation of the quantized speed output by the TA shown in figure 40 . The elevator waits in state Braking for the quantized speed $q$ value to reach zero. Once the 
elevator speed reaches zero, the transition from Braking to Stopped is enabled and executed, and then, the elevator sends a synchronization signal on the channel stop! to the elevator-controller to let it know it has stopped. The rest of the model executes exactly as shown before in section 4.8. This model of the originally hybrid system allows the designer to verify the control system with different parameters of the elevator's physical system, such as different braking values of de-accelerations, different elevator initial speeds, or other parameters in a more detailed QSS model. This is an important addition to the elevator system verification as relevant physical factors to the controller performance can be identified and formally verified during the design phase.

In section 4.8 , we showed how to verify a number of desired properties for the DEVS model (such as deadlock freedom, bounded response time, and safety properties) for the elevator coupled model. We used Computational Tree Logic (CTL) to construct queries with the requirements and submitted it to UPPAAL to get an answer and hence verify that requirement. Here we show how to check one of these required properties here with the hybrid system modeled in the previous section. We start with an elevator model as the one described in figure 41 with its speed decrease as in figure 39.

One such requirement is the freedom of deadlocks expressed in CTL as

A[] not deadlock.

This means for all paths, there should be no deadlocks.

After running the checker, it shows that this property is satisfied, i.e. there is no deadlock in the DEVS model: 
(Academic) UPPAAL version 4.0.13 (rev. 4577), September 2010 -- server.

A[] not deadlock

Property is satisfied.

In another example, a second elevator with braking de-acceleration equals $\left(-0.12 \mathrm{~m} / \mathrm{s}^{2}\right)$ has its continuous and quantized speeds described by graphs shown in figure 42 and figure 43 .

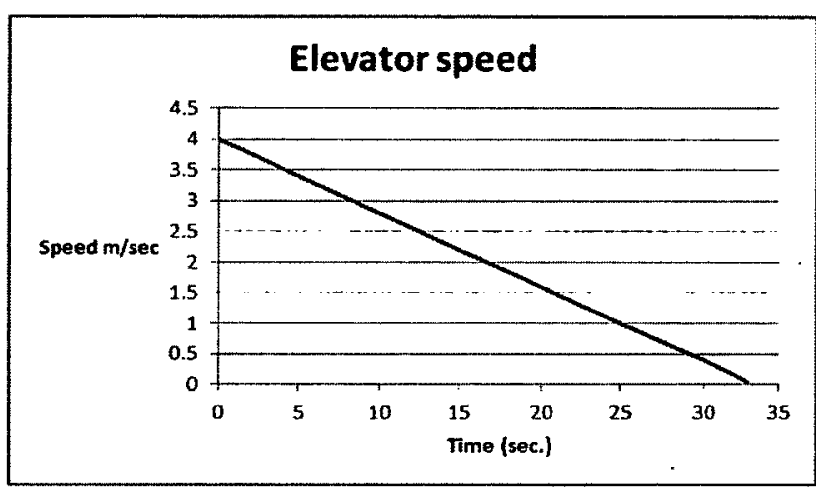

Figure 42: Elevator speed, acceleration $=-0.12 \mathrm{~m} / \mathrm{sec}^{2}$.

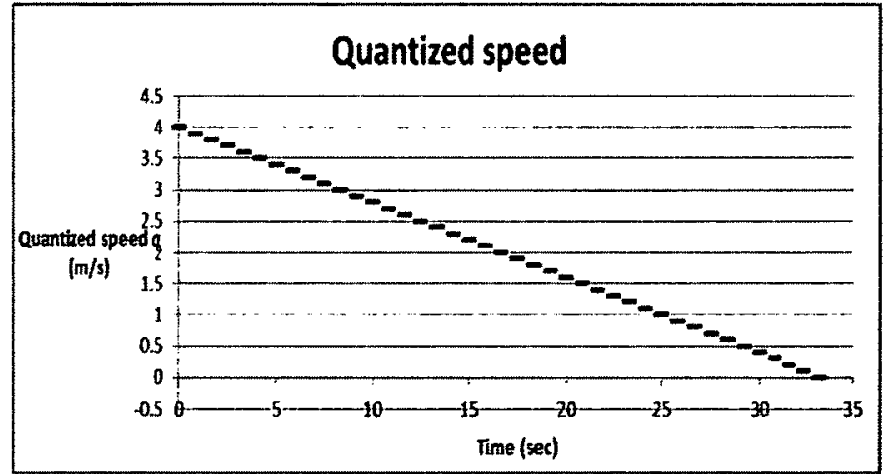

Figure 43: Quantized speed, acceleration $=-0.12 \mathrm{~m} / \mathrm{s}^{2}$. 
To verify the elevator-controller with this second version, we changed the parameters of the elevator acceleration in its TA model and re-verified again. The results are shown as follows:

A[] not deadlock
Property is not satisfied.

In this case, the time needed for the elevator to stop is approximately 33 seconds. This would contradict with the user requirements model shown in figure 29. In this model, the user expects the elevator to reach $3^{\text {rd }}$ floor within 27 seconds at most, and after this time the requirement for the elevator controller to be ready to accept another as shown on the transition $S 5 \rightarrow S 6$. However, the slow-braking elevator would not be able to fulfill the second request in time, hence we have a time lock [77] and the model cannot progress beyond $S 5$.

We add a note here about deadlock verification. Deadlock freedom is an example of liveness property. A system deadlock means the non-existence of enabled transitions in the model, i.e. lack of system behavior at this point in time. When we over-approximate, we add some extra behaviour to the model. Thus verification of deadlock freedom on the over-approximated model does not guarantee that the original model is also deadlockfree. This is due to the uncertainty if the deadlock freedom is a result of the original system behaviour, or the extra behaviour added in the over-approximation. However, if the over-approximated model shows a deadlock, this guarantees the original system has also a deadlock as the original system has less behaviour. This result is because liveness property in general cannot be expressed as a safety property as shown in [80]. This 
limitation does not affect our methodology as the bounded liveness property is of the most importance to a real-time system. Knowing that a response would come eventually in future (as deadlock freedom, and general liveness properties imply) is not enough for real-time system correctness, we need to guarantee a bounded-delay for a response, for the system to be useful. Bounded liveness property can be expressed as a safety property, and hence preserved in the over-approximation [80].

\subsubsection{Verifying general QSS}

To verify a general QSS system as defined above in section 2.4.1 using timed automata, it is necessary to preserve all the important properties that we may wish to verify (such as safety, reachability, and bounded liveness). To do so, we use an overapproximation model to contain all system behaviours defined in the QSS model as we

shown before. In doing so, we need to convert all real numbers with fractional parts to integers. This is typically done by changing the model scale by multiplying all constants by the least common denominator. However, this can only be done at the model design time for the quantization levels and the quantum values, as we know the set of quantization levels and the value of the quantum before model execution. There are other computed value of the timed automata model such as the state lifetime $\sigma$, and the intermediate values of $x$ and $f(x)$. These values are computed during the model's execution, thus we have no idea about these values beforehand, and so we cannot determine a common denominator for all the possible values computed during runtime. To overcome this, we need to specify a timed automata model that allows an event to 
occur at any possible time between $\lfloor\sigma\rfloor$ and $\lceil\sigma\rceil$, or in case of another variable $x$, the true variable value would be in a closed interval $[\lfloor x\rfloor,\lceil x\rceil]$. This effectively gives us an overapproximation model to the QSS that includes all possible behaviours of the original QSS model.

We show in more detail this over-approximation with the exponential decay formula we introduced in section 2.4.1. This formula has the analytical solution $x(t)=e^{-t}$, with the initial condition $x(0)=1$. Figure 44 shows a graph of the exact analytical solution of the exponential decay formula $x(t)=10 e^{-t}$ where $x(0)=10$.

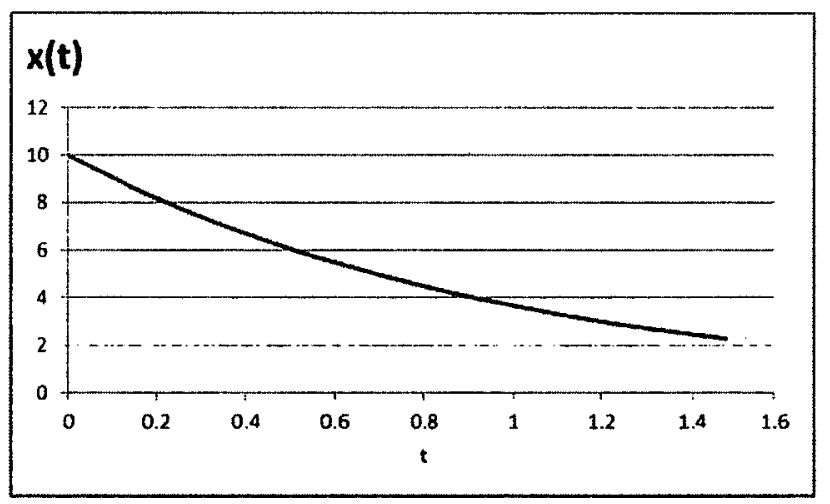

Figure 44: Exact solution for exponential decay formula.

QSS approximates the continuous system with linear segments, and generates events whenever the approximated solution crosses a quantization level. Figure 45 shows the QSS approximate solution versus the exact solution of the decay formula. 


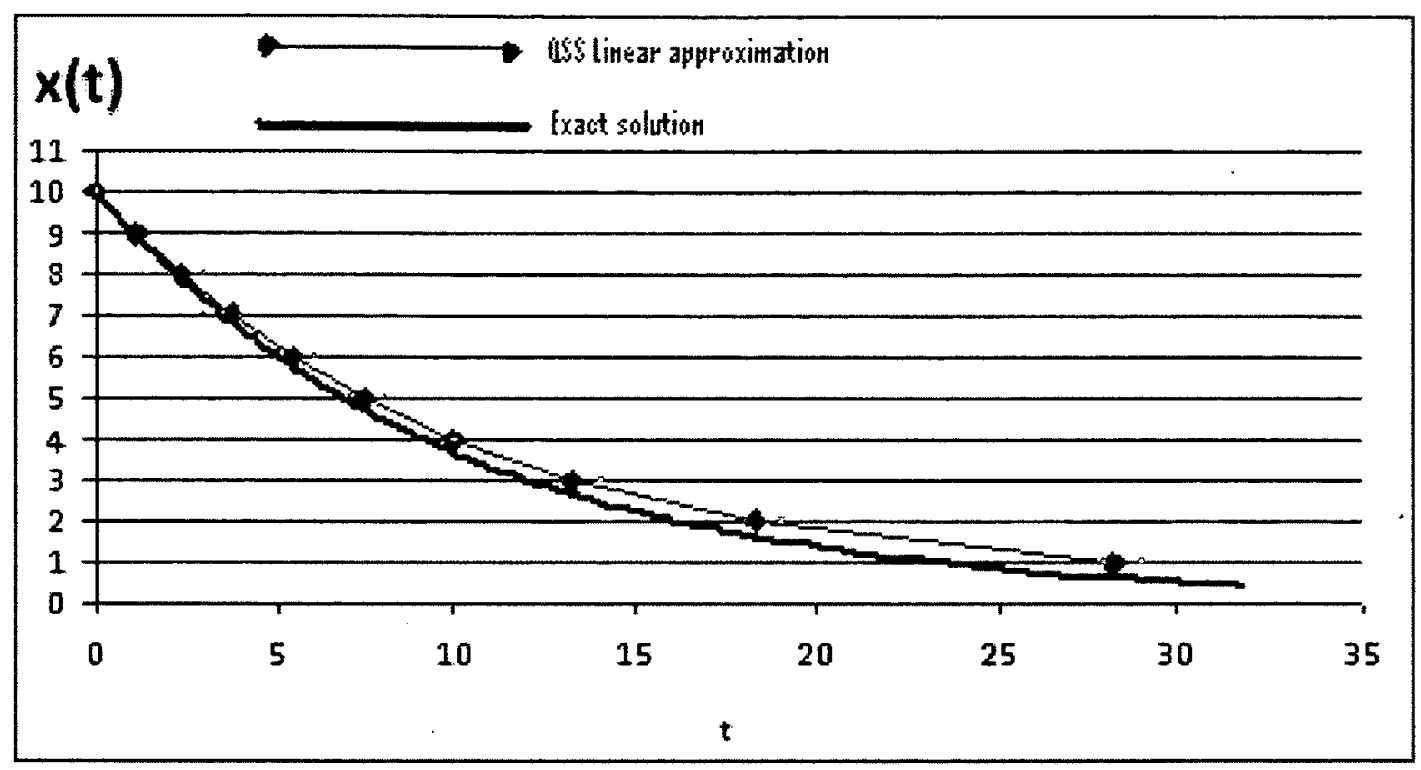

Figure 45: QSS linear approximation

Figure 46 shows events generated by QSS based on the approximate solution. These events represent the system state changes at points in time. In that figure, continuous line curve represents the exact solution; stepped (marked with $\mathrm{x}$ ) graph represents QSS approximation where sigma is calculated as Real number. The stepped graph, marked with $\mathrm{F}$, and the one marked with $\mathrm{C}$, represents the over-approximation of QSS graph by $\lfloor\sigma\rfloor$ and $\lceil\sigma\rceil$ respectively. The time scale has been rescaled by multiplying by 10 to show details. This figure shows that the QSS behaviour, defined as pairs (event $t_{i}$, time $_{i}$ ), is contained within the over-approximated model. 


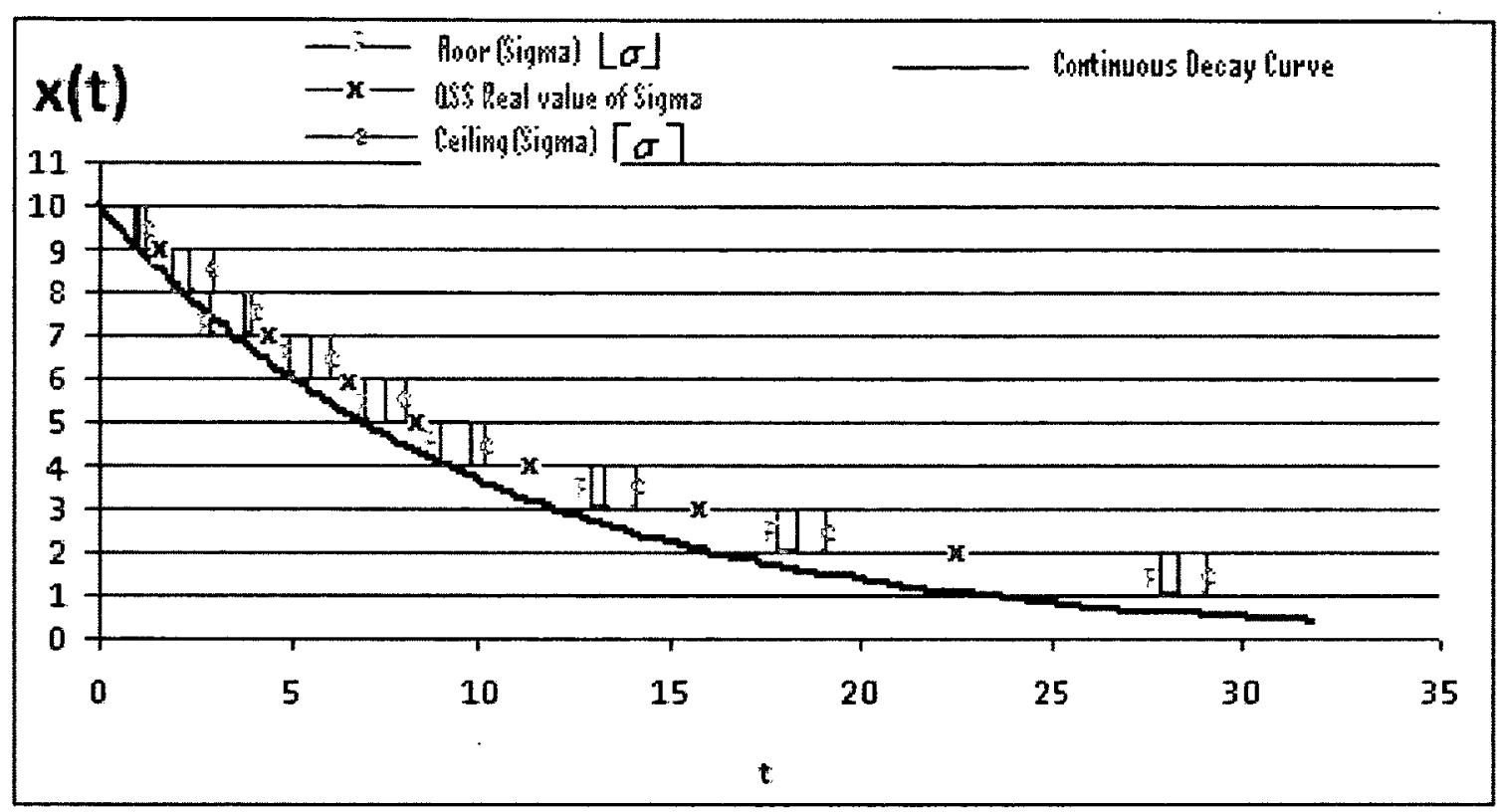

Figure 46: Over-approximation of QSS trace.

To show the effect of the over-approximation for preserving the safety property, we assume that we want to verify the QSS system in regard to some safety conditions for its state variable. These conditions are that the value of the system state variable $x$ would not fall into an unsafe zone (such as zonel and zone2 as shown in figure 47). From the diagram, we see that if the over-approximation is safe concerning zone 1, the actual QSS trajectory is guaranteed to be safe as well. The over-approximation however, can show the system is not safe in regard to zone 2 as the state trajectory enters zone 2 at $(x=3,17 \leq t$ $\leq 18$ ). In this case, we can see that the actual QSS system is safe as the actual QSS trajectory does not intersect with zone2. This is a typical spurious violation of safety due to the added behaviour with over-approximation; hence over-approximation provides a more conservative model for safety verification. 
We note here a limitation of the QSS method to model the actual system trajectory. As the QSS method in this example uses linear approximation, it is known to have an approximation error when simulating continuous systems with nonlinear dynamics as shown in [51]. This approximation error is inversely proportional to the quantum value $d Q$. However, by reducing $d Q$, the number of iterations increases. Some extensions for the QSS method are using a second-order equation to approximate continuous systems [60], which results in the ability to reduce $d Q$ without much increase in the number of iterations. This limitation however, is part of the QSS method, and we do not try to remove this limitation in this research. We just verify the QSS model that the modeller chooses to simulate, and verifies. In doing so, the modeller is aware with the small approximation due to QSS method, and it is acceptable for his/her purpose. In future work, addressing the gap between QSS trace, and the actual system trajectories and its effect to formal verification can be done. We expand on this point in the future work section in section 6.1.1.

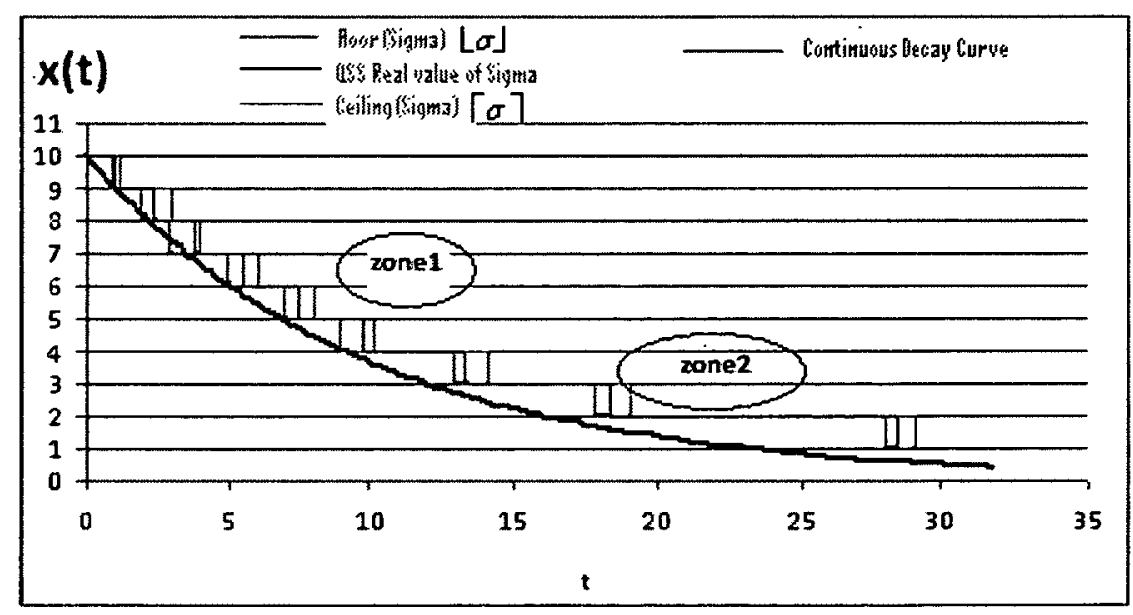

Figure 47: Safety zones with QSS over-approximation.

132 
To extend these ideas to a general QSS system, a major obstacle to model general QSS models with TA is that QSS uses variables of type Real, while TA has only Integer type variables. This necessitates an approximation of all Real-valued variables in QSS to an equivalent Integer values. When faced with obtaining an Integer approximation to a Real value, we need to round the Real value to nearest Integer. However, in doing so, we lose the actual value as it is in the QSS model, and this may render TA verification results not applicable to the original QSS system.

To include the actual value of the QSS variables in our approximation, we approximate any QSS Real-value with a closed interval between two Integers [L,U], where $\mathrm{L}$ is the mathematical floor function of the Real-value, and $\mathrm{U}$ is the mathematical ceiling. This would constitute an over-approximation that contains the true trajectory produced by the QSS. This over-approximation, when transformed to a TA model, would contain all possible behaviours in the QSS model. Thus it would simulate the original QSS system. In the resulting over-approximation QSS model, any calculations would use interval arithmetic as introduced in section 2.5.

- To show this, we construct a QSS model where only integers and integer intervals are used. Thus, the QSS system of (Eq. 2.6) on page 37 may be written as:

$$
M_{1 O A}=\left(X, Y, S, \delta_{\text {int }}, \delta_{\text {ext }} \lambda,\right. \text { ta), where }
$$

$$
\begin{aligned}
& X=\mathrm{Z}^{2} \times\{\text { inport }\} \\
& Y=\mathrm{Z} \times\{\text { outport }\}
\end{aligned}
$$




$$
\begin{gathered}
S=Z^{4} \times Z \times Z^{2+}{ }_{0} \infty \\
\delta_{\text {int }}(s)=\delta_{i n t}\left(\left[x_{L}, x_{U}\right],\left[d_{x L}, d_{x U}\right], k,\left[\sigma_{L}, \sigma_{H}\right]\right)= \\
\left(Q_{k+l},\left[d_{x L}, d_{x U}\right], k+\operatorname{sgn}\left(d_{x L}\right),\left[\sigma_{I L}, \sigma_{I H}\right]\right) \\
\delta_{e x t}\left(s, e, x_{u}\right)=\delta_{e x t}\left(x, d_{x}, k, \sigma, e, x_{v}, p o r t\right)= \\
\left(\left[x_{L}, x_{U}\right],\left[x_{v L}, x_{v L}\right], k,\left[\sigma_{2 L}, \sigma_{2 H}\right]\right) .
\end{gathered}
$$

$\lambda(s)=\lambda\left(x, d_{x}, k, \sigma\right)=\left(Q_{k+\operatorname{sgn}(d x)}\right.$, outport $)$

$$
\operatorname{ta}(s)=\operatorname{ta}\left(x, d_{x}, k, \sigma\right)=\left[\sigma_{L}, \sigma_{H}\right]
$$$$
x_{L}=x+e \cdot d_{x L}
$$

$$
x_{U}=x+e \cdot d_{x U}
$$

$\sigma_{I L}= \begin{cases}\frac{Q_{k+2}-\left(Q_{k+1}\right)}{d_{x U}} & \text { if } d_{x U}>0 \\ \frac{Q_{k}-\left(Q_{k-1}-\varepsilon\right)}{\left|d_{x U}\right|} & \text { if } d_{x U}<0 \\ \infty & \text { if } d_{x U}=0\end{cases}$

$$
\sigma_{l U}= \begin{cases}\frac{Q_{k+2}-\left(Q_{k+1}\right)}{d_{x L}} & \text { if } d_{x L}>0 \\ \frac{Q_{k}-\left(Q_{k-1}-\varepsilon\right)}{\left|d_{x L}\right|} & \text { if } d_{x L}<0 \\ \infty & \text { if } d_{x L}=0\end{cases}
$$

(Eq. 5.7)

134 


$$
\begin{gathered}
\sigma_{2 L}= \begin{cases}\frac{Q_{k+1}-\left(x_{L}+e . d_{x L}\right)}{x_{v L}} & \text { if } x_{v L}>0 \\
\frac{\left(x_{L}+e . d_{x L}\right)-\left(Q_{k}-\varepsilon\right)}{\left|x_{v L}\right|} & \text { if } x_{v L}<0 \\
\infty & \text { if } x_{v L}=0\end{cases} \\
\sigma_{2 U}= \begin{cases}\frac{Q_{k+1}-\left(x_{U}+e . d_{x U}\right)}{x_{v L}} & \text { if } x_{v U}>0 \\
\frac{\left(x_{U}+e . d_{x U}\right)-\left(Q_{k}-\varepsilon\right)}{\left|x_{v U}\right|} & \text { if } x_{v U}<0 \\
\infty & \text { if } x_{v U}=0\end{cases}
\end{gathered}
$$

and from (Eq. 2.4) on page 34, we calculate current

function slope as:

$$
\begin{aligned}
& d_{x L}=\lfloor f[q(t), u(t)]\rfloor \\
& d_{x U}=\lceil f[q(t), u(t)]] \\
& x_{v L}=\left\lfloor f\left[x_{\mathrm{L}}(t), u(t)\right]\right\rfloor \\
& x_{v U}=\left\lfloor f\left[x_{\mathrm{U}}(t), u(t)\right]\right\rfloor
\end{aligned}
$$

In the model of (Eq.5.6),(Eq. 5.7),(Eq. 5.8),(Eq. 5.9) we replaced every computed Real-valued quantity with a bounded Integer interval. Formally, for any calculated Realvalue $r$, we replace it with a closed interval $[\lfloor r\rfloor,\lceil r\rceil]$. This makes the model of (Eq.5.6),(Eq. 5.9) an over-approximation to the QSS model of (Eq. 2.6) on page 37. Thus, it would contain all behaviours of (Eq. 2.6) model, along with some added behaviour due to the over-approximation. Preservation of behaviour into the over-approximation is vital to 
verification. As this guarantees that any safety, and bounded liveness property that is verified in (Eq.5.6)-(Eq. 5.9) model, would also be true for (Eq. 2.6) model.

We also note here that in replacing Real-valued quantities with integer intervals, we did not need to do this for quantization levels $Q=\left\{Q_{0}, Q_{1}, \ldots Q_{n}\right\}$, quantum value $d Q$, or hysteresis value $\varepsilon$, assuming all these values are rational numbers. These values are parameters of any QSS model and are known before runtime, thus these values can be represented as integers by changing the model scale by multiplying all these numbers by least-common-multiple denominator as we described in chapter 3.

Another obstacle in representing general QSS models with TA is the nature of the function $f$ of (Eq. 2.4) on page 34. In UPPAAL, users can define functions with a syntax close to that of $\mathrm{C}$ language. However, the available operators are limited to the primary mathematical operators, as shown in table 3. Furthermore, operands and expression results are all integers. This puts a limit on the functions that can be expressed in UPPAAL TA. Similar restrictions on the system dynamics also exist with other formalisms for hybrid systems verification such as Linear Hybrid Automata [82], where derivatives are limited by linear constraints. To model QSS systems whose dynamics are described by complex function, the modeller would need to approximate the complex function with a polynomial formula that uses only preliminary operators of UPPAAL. This approximation may use one of the series expansion methods such as the Maclaurin Series, and can be done up to the desired precision. In this case, the system function 
would have to be differentiable and the types of functions we can use are limited by Maclaurin series limitations. However, it should be noted that the more precision we get, the higher values are obtained for constants in the resulting TA model and hence more resources (memory and time) would be needed to verify the model as we discuss in section 6.1.1. This approximation, however, is out of the scope of this thesis.

Table 3: UPPAAL arithmetic operators

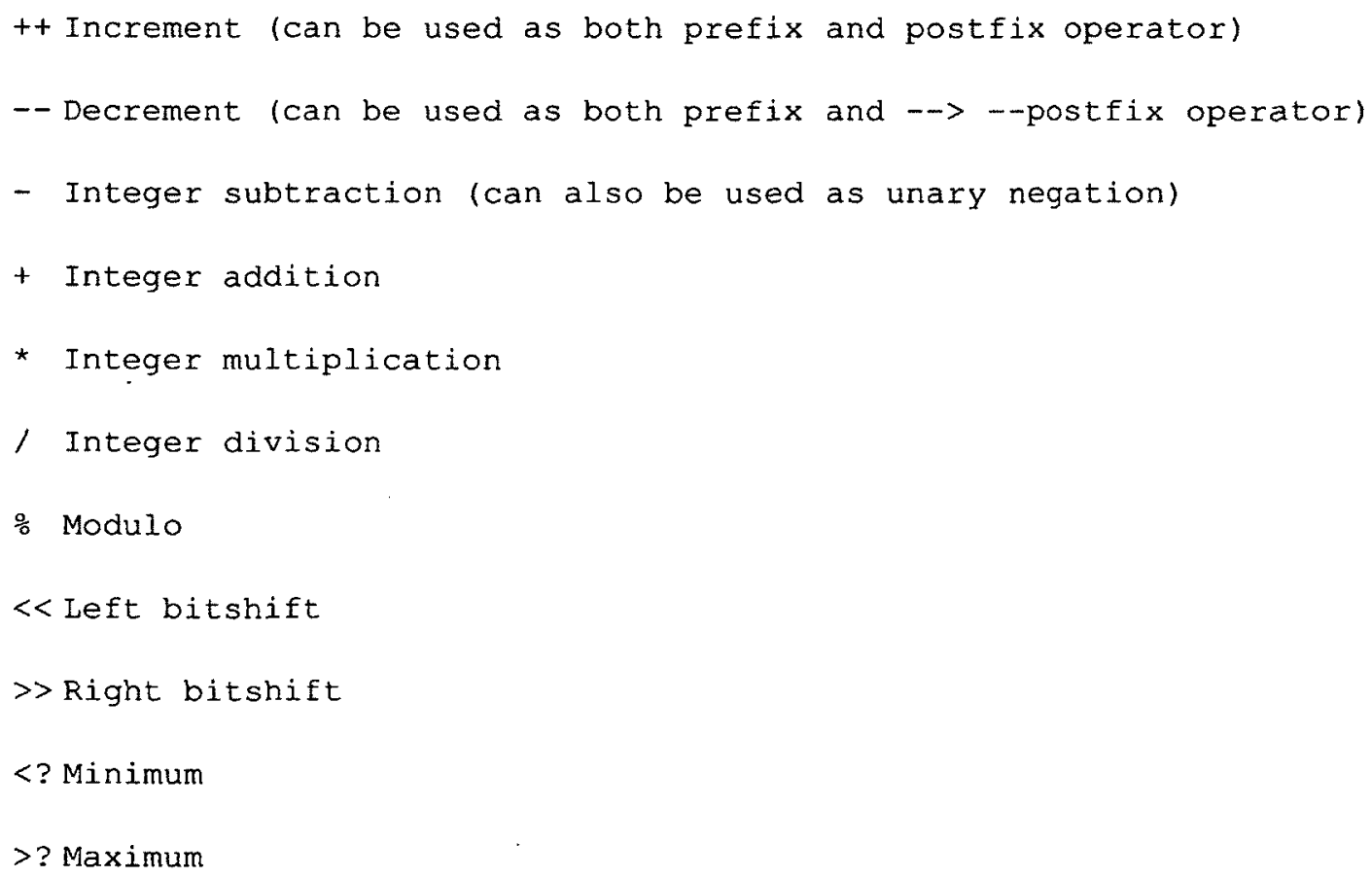

The integrator automaton representing the QSS model of (Eq.5.6) is shown in figure 48. The structure of this automaton would be fixed for any QSS system, and thus can be considered as a template representing the DEVS model of (Eq.5.6). This template has the following parameters that are part of the QSS model definition: 
- A set of defined quantization levels $Q=\left\{Q_{0}, Q_{l}, \ldots . Q_{r}\right\}$, where $Q_{0}$ is the initial level, $Q_{r}$ is the final level.

- The quantum $d Q=\mathrm{Q}_{\mathrm{k}+1}-\mathrm{Q}_{\mathrm{k}}$, such that $0 \leq k<r$

- Hysteresis value $\varepsilon$

- System defined functions: $f[x(t), u(t)], x(t)$, and $u(t)$.

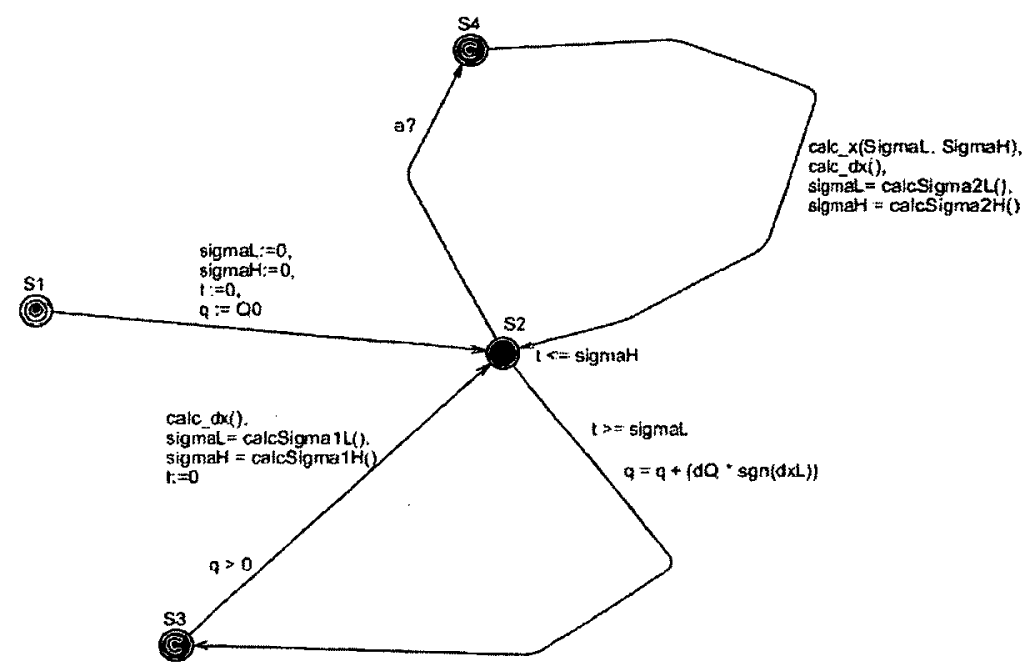

Figure 48: TA model of a QSS general Integrator

This automaton starts in S1, and on the transition from $\mathrm{S} 1$ to $\mathrm{S} 2$, it initializes the variables sigmaL, sigmaH, $q$, and clock t. The internal transition function $\delta_{m}(s)$ of (Eq.5.6) is simulated with transitions $\mathrm{S} 2 \rightarrow \mathrm{S} 3$, and S3 $\rightarrow \mathrm{S} 2$. At $\mathrm{S} 2$, the automaton waits for a time $t$, where $\sigma_{I L} \leq t \leq \sigma_{I H}$, then transits to S3. On this transition, value of $q$ is updated as $Q_{k+\operatorname{sgn}(d x)}=q+\left(d Q^{*} \operatorname{sgn}\left(d_{x}\right)\right)$, which is equivalent to the expression $(x+\sigma$. $\left.d_{x}\right)$ of (Eq. 2.6) on page 37. Then, on transition $\mathrm{S} 3 \rightarrow \mathrm{S} 2$, an updated values of $d_{x \mathrm{~L}}, d_{\mathrm{xU}}$ are calculated according to (Eq. 5.9), then values $\left[\sigma_{I L}, \sigma_{I H}\right]$ are calculated according to 138 
(Eq. 5.7). Clock $\mathrm{t}$ is also reset to zero on the transition $\mathrm{S} 3 \rightarrow \mathrm{S} 2$ before waiting in $\mathrm{S} 2$ for the next event. The output function $\lambda(s)$ is implicit in the TA model, as the value of shared variable $q$ can be read by other models coupled with this TA model. The definitions of UPPAAL functions of these calculations are shown in table 4.

To simulate the external transition function $\delta_{e x t}\left(s, e, x_{w}\right)$ of (Eq.5.6), we use the transitions S2 $\rightarrow \mathrm{S} 4 \rightarrow \mathrm{S} 2$. Transition S2 $\rightarrow$ S4 would be enabled only if the automaton receives a synchronization input on channel a. This is typically another automaton generating the function $u(t)$ as shown in figure 49 , and would be described later. When this synchronization happens, the automaton moves to the committed state $\mathrm{S} 4$, and then, without delay, it executes the transition $\mathrm{S} 4 \rightarrow \mathrm{S} 2$. On this latter transition, the new values of $\left[x_{L}, x_{U}\right]$ are calculated based on the shared variables SigmaL, SigmaH which are passed from the automaton of figure 49 . New values of $\left[x_{v L}, x_{v L}\right]$ are also calculated which represents the new slope value. Finally, new values for $\left[\sigma_{2 L}, \sigma_{2 H}\right]$ are calculated. The UPPAAL functions that we defined for these calculations are shown in table 5. 


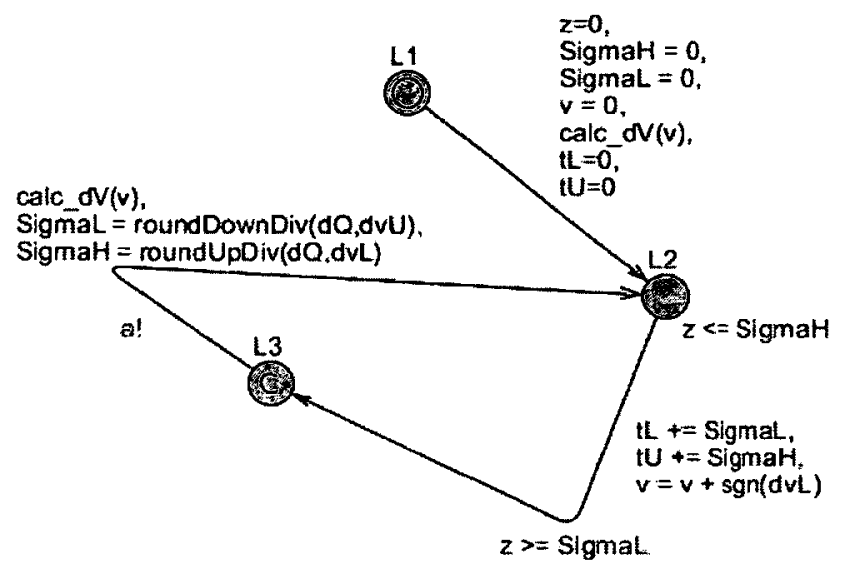

Figure 49: TA Model of a QSS Input function generation 
Table 4: User defined functions for the UPPAAL TA model of figure 48

\begin{tabular}{|c|c|c|}
\hline int sgn(int $\mathrm{dx}) \mathrm{l}$ & $\begin{array}{l}\text { void calc_dx()/ } \\
\text { //calculates //the slope } \\
\text { from system //defined } \\
\text { function } \\
d x L=f(x L, v) ; \\
d x U=f(x U, v) ;\end{array}$ & $\begin{array}{l}\text { int flint } x, \text { int } v \text { ll } \\
\text { //calculates the slope from } \\
\text { //system defined function } \\
/ / \text { Replace } f \text { with system } \\
/ / \text { function here... } \\
\text { int } f=x+v \text {; }\end{array}$ \\
\hline \multicolumn{3}{|c|}{ 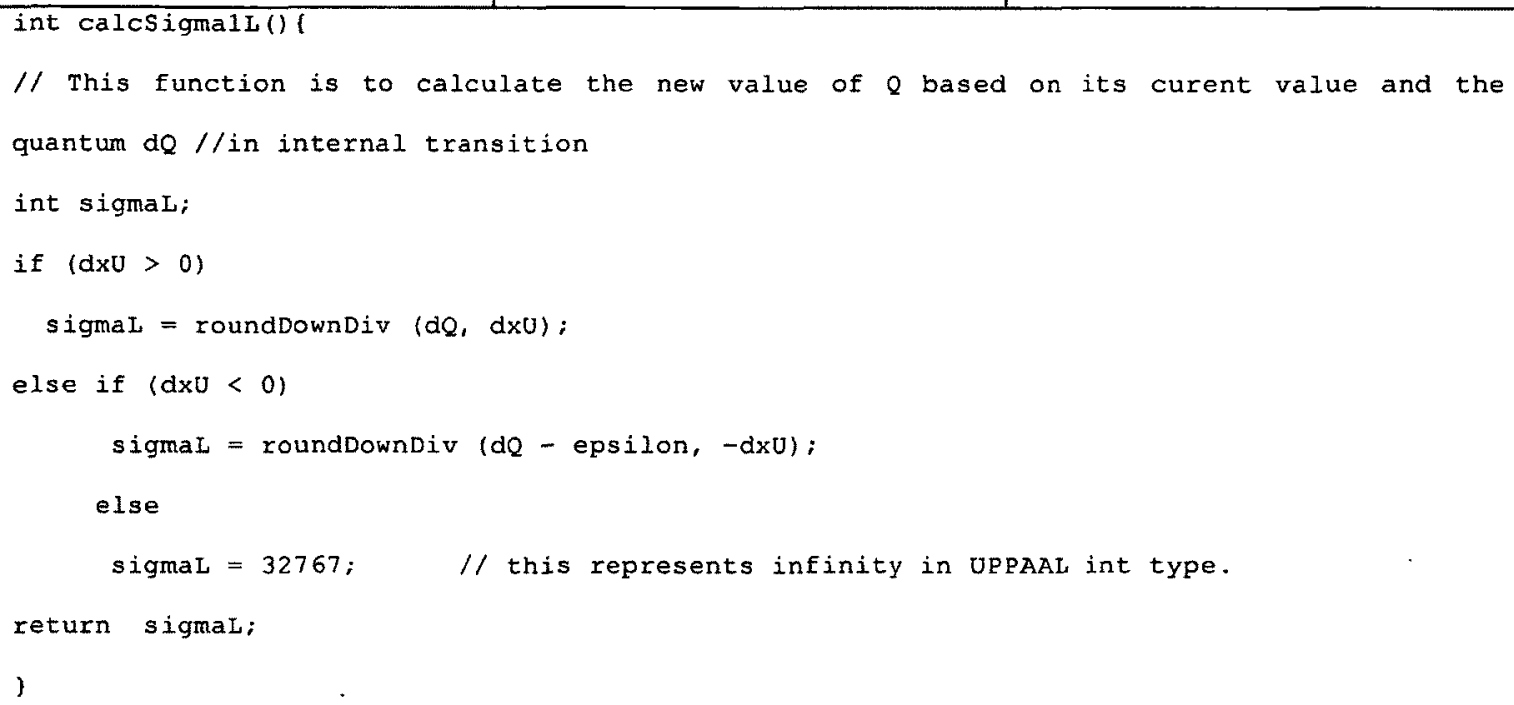 } \\
\hline $\begin{array}{l}\text { int calcsigma1H() } \\
/ / \text { This function is } \\
\text { quantum } d Q / / \text { in inter } \\
\text { if }(d x L>0)\end{array}$ & $\begin{array}{l}\text { te the new value of } Q \text { based } \\
\text { tion } \\
\text { epsilon, }-d x L) \text {; }\end{array}$ & 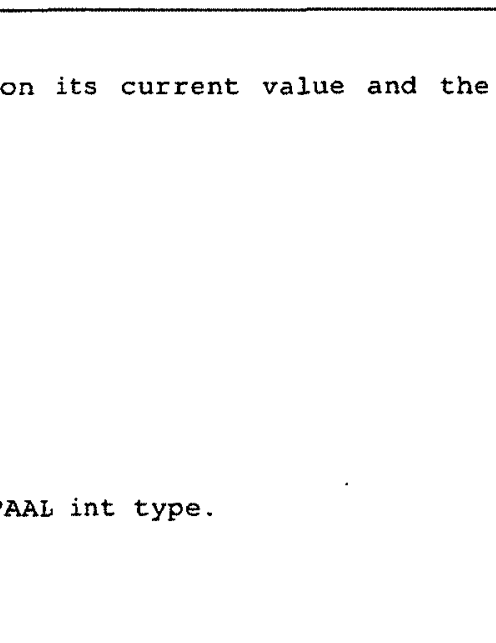 \\
\hline
\end{tabular}


Table 5: User defined functions for the UPPAAL. TA model of figure 49

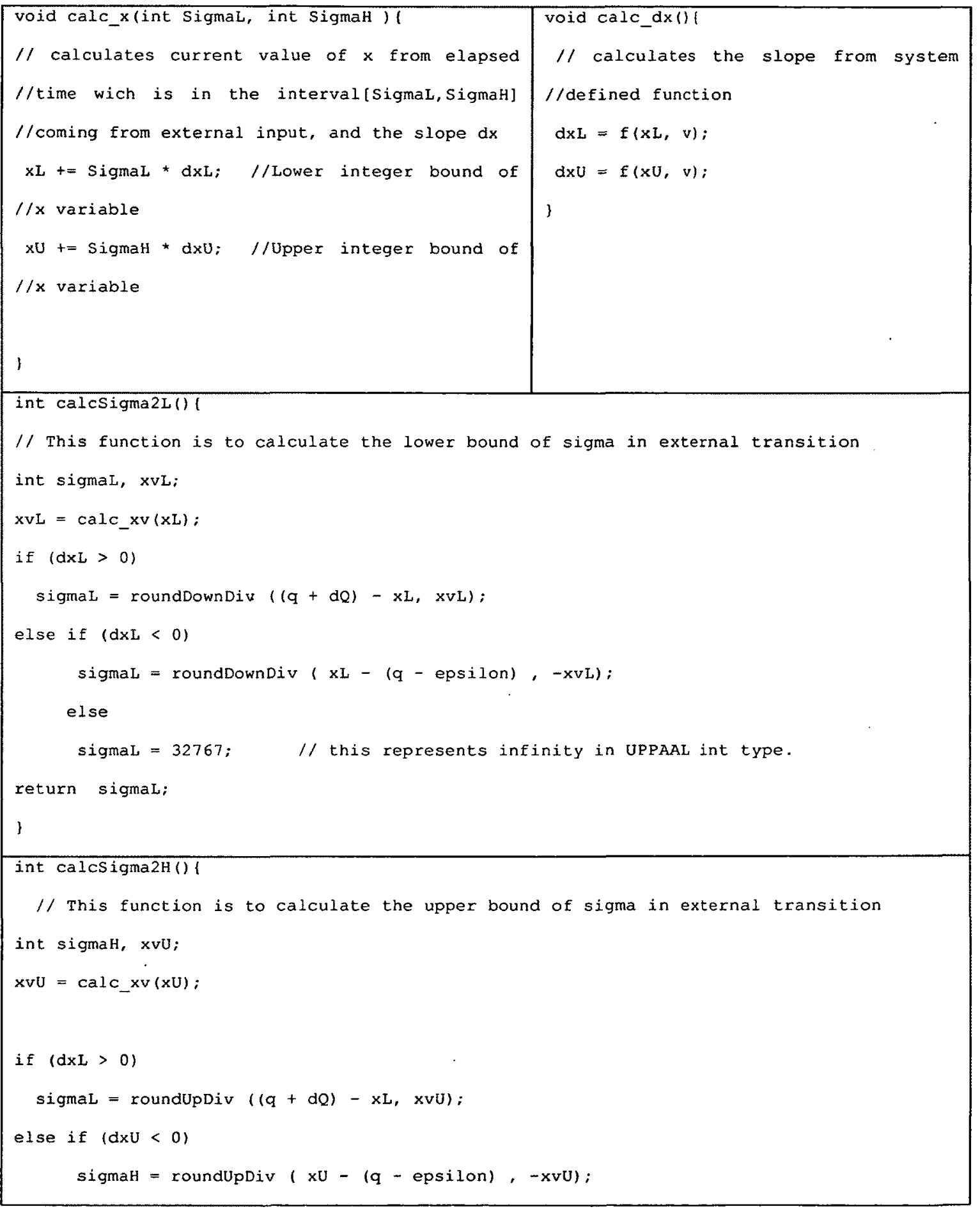




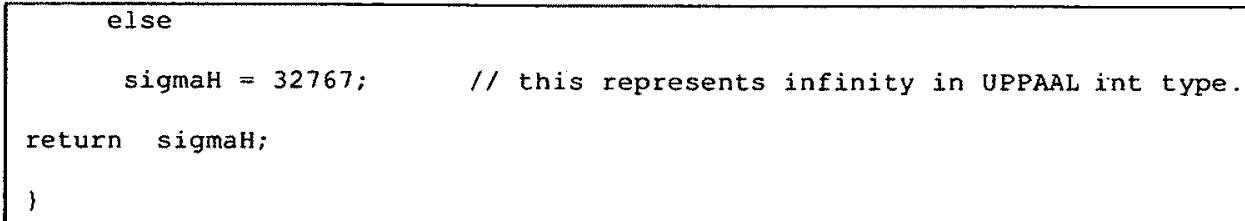

For a general QSS system where $u(t) \neq \varphi$, the QSS model would have an external transition function to process the input $u(t) . u(t)$ is a constant input step function as defined in QSS [51]. To simulate a system described with ODE's as (Eq. 2.4) on page 34, the input $u(t)$ needs to be generated by a DEVS QSS model. One example is given in [60] for a sinusoidal shape input signal. This DEVS QSS model is similar to the model defined in (Eq.5.5), as it does not have external inputs, and it generates a sequence of quantized events to represent the step function $u(t)$. Such a system can be described by an óver-approximated QSS model Mu $\mathrm{OA}_{\mathrm{A}}$ where we use closed integer intervals instead of Real numbers, for the same reason we did with the integrator QSS model above. The over-approximated model of the input function is shown in (Eq. 5.10) - (Eq. 5.12).

$$
M u_{O A}=\left(X, Y, S, \delta_{\text {int }}, \delta_{\text {ext }}, \lambda, \text { ta }\right), \text { where }
$$

$$
\begin{aligned}
& X=\varphi \\
& Y=\mathrm{Z} \times\{\text { outport }\} \\
& S=\mathrm{Z}^{4} \times \mathrm{Z} \times \mathrm{Z}^{2+}{ }_{0} \infty \\
& \delta_{\text {int }}(s)=\delta_{\text {int }}\left(\left[u_{L}, u_{U}\right],\left[d_{u L}, d_{u U}\right], k,\left[\sigma_{L}, \sigma_{H}\right]\right)=
\end{aligned}
$$




$$
\left(Q_{k+1},\left[d_{u L}, d_{u U}\right], k+\operatorname{sgn}\left(d_{u L}\right),\left[\sigma_{I L}, \sigma_{I H}\right]\right)
$$

$\delta_{e x t}\left(s, e, x_{w}\right)=\varphi$

$\lambda(s)=\lambda\left(x, d_{\iota}, k, \sigma\right)=\left(Q_{k+\operatorname{sgn}(d u),}\right.$ outport $)$

$\operatorname{ta}(s)=\operatorname{ta}\left(x, d_{u}, k, \sigma\right)=\left[\sigma_{L}, \sigma_{H}\right]$

$\sigma_{I L}= \begin{cases}\frac{Q_{k+2}-\left(Q_{k+1}\right)}{d_{u U}} & \text { if } d_{u U}>0 \\ \frac{Q_{k}-\left(Q_{k-1}-\varepsilon\right)}{\left|d_{u U}\right|} & \text { if } d_{u U}<0 \\ \infty & \text { if } d_{u U}=0\end{cases}$

(Eq. 5.11)

$\sigma_{I U}= \begin{cases}\frac{Q_{k+2}-\left(Q_{k+1}\right)}{d_{u L}} & \text { if } d_{u L}>0 \\ \frac{Q_{k}-\left(Q_{k-1}-\varepsilon\right)}{\left|d_{u L}\right|} & \text { if } d_{u L}<0 \\ \infty & \text { if } d_{u L}=0\end{cases}$

and current function slope as:

(Eq. 5.12)

$d_{u L}=\lfloor u(t)\rfloor$

$d_{u U}=\lceil u(t)\rceil$

In this QSS model, system state is defined with a tuple (value of variable $u$, slope of state variable $d_{v}$, current quantization level index $k$, and state lifetime $\sigma$ ). However, as we need to use only Integers to represent these quantities, we replaced each one with a closed 
integer interval. The integer interval is chosen to include inside it the quantity we are approximating. We do this by choosing the lower bound of the interval with the Floor function of the quantity, and the upper bound to be the Ceiling function. Thus the corresponding intervals to describe the QSS state would be $\left(\left[u_{L}, u_{U}\right],\left[d_{u L}, d_{u U}\right], k,\left[\sigma_{L}\right.\right.$, $\left.\sigma_{H}\right]$ ). As this model has no external input, then we know, from the semantics of QSS method, that the state variable $u$ would always take a value from the set $Q$ of the quantization levels. Thus the term $\left[u_{L}, u_{U}\right]$, of the state, would be the current quantization level $\mathrm{Q}_{k+1}$. Calculation of the state lifetime $\sigma$, is done with arithmetic intervals in (Eq. 5.11) as the slope of the function is expressed as an interval, which in turn is calculated in (Eq. 5.12).

We follow the same steps we did for the QSS model of the integrator above to simulate this QSS model with timed automata, resulting in the TA model shown in figure 49. The transition $\mathrm{L} 1 \rightarrow \mathrm{L} 2$ initializes clock $\mathrm{z}$, the lower and upper time constraints $\left[\sigma_{I L}\right.$ ,$\left.\sigma_{l H}\right]$, the quantized output variable $v$, and calculates the initial slope from the function definition of $u(t)$ by invoking user defined function calc_dV(v). The slope is calculated as a closed integer interval $[d v L, d v U]$ to represent the over-approximation of slope value, as defined in (Eq. 5.12).

$\delta_{\text {im }}(s)$ is simulated by transitions $\mathrm{L} 2 \rightarrow \mathrm{L} 3 \rightarrow \mathrm{L} 2$. On these two transitions, new value of $v$ is calculated, the function calc_dV(v) calculates the values of $u(t)$, then the new values of next event timing is calculated $\left[\sigma_{I L}, \sigma_{I H}\right]$. We note here that to calculate $u(t)$ we need the 
current value of time $t$. At any time during the model execution, the total elapsed time $t$ after $j$ number of internal transitions is given by:

$t_{j}=\sum_{0 \leq i \leq j-1} \sigma_{i}$

And as we have an estimate of $\sigma$ in the interval $\left[\sigma_{I L}, \sigma_{I H}\right]$, we get:

$$
t_{j_{L}}=\sum_{0 \leq i \leq j-1} \sigma_{i L} \quad, \quad t_{j U}=\sum_{0 \leq i \leq j-1} \sigma_{i U}
$$

This gives an estimate of time at current iteration $j$ as shown on transition $\mathrm{L} 2 \rightarrow \mathrm{L} 3$ with the integer interval $\left[t_{L}, t_{U}\right]$.

On the transition from L3 to L2, after the automaton calculates the next values of shared variables SigmaL and SigmaH, it synchronizes on channel "a" to the integrator automaton shown in figure 48 , so the latter can read the values of $v$, SigmaL, and SigmaH which are used in the external transition definition to recalculate a new function slope $x_{v}$ as per the QSS model of (Eq.5.6).

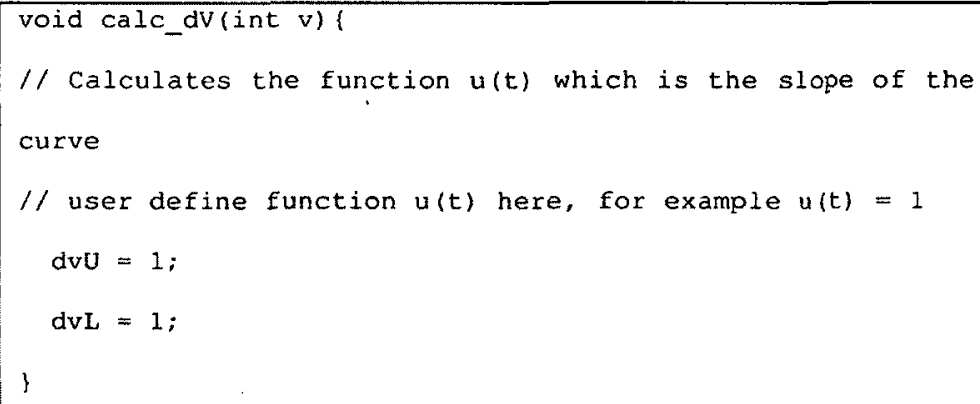

Figure 50: Definition template of $u(t)$ in UPPAAL. 


\subsubsection{Advantages of using QSS/DEVS to verify Hybrid Models}

Enabling approximation to system dynamics in timed automata models using QSS opens the door to more complex verification queries and better controller designs. For example, the following research directions can be built on results of hybrid systems verification using QSS, as we introduced earlier in this chapter:

- System dynamics are presented in a fine-grain in the TA model. This would enable verification of more advanced types of controllers than an On-Off controller. For example, advanced control algorithms could use the information about the state variable change with respect to time.

- Optimization controller designs could also be verified in UPPAAL based on some optimization constraints on the values of state variables.

- TA controller synthesis techniques could use the fine-grained information of system dynamics to synthesis advanced controllers based on QSS environment models. 


\section{Chapter 6: Conclusion \& Future Research}

In this thesis, we have presented a methodology for the verification of different DEVS models. This methodology covers different components, including both deterministic classic DEVS and continuous components, which were modeled as DEVS using the QSS method (the QSS method was selected as the way to discretize the continuous behaviour using DEVS; however, other current or future methods within DEVS could be used in a similar fashion).

The contributions of this work include a verification methodology for deterministic classic DEVS. Although, as identified in the State of the Art section, other groups have used TA to verify DEVS in parallel with our research, the work introduced in this research is different. The main contribution is the provision of a complete method that deals with the issues that prevent a full verification of DEVS models as shown in section 4.1. It also provides a formal methodology to convert DEVS models into behaviourally equivalent TA models. Finally, the methodology provides an estimation method for any errors that could have occurred due to the conversion and the approximation process. Hybrid DEVS verification presented in this thesis is also an original result. These contributions resulted in numerous publications, listed in section 3.1. 


\subsubsection{Possible Future Research}

This dissertation lays the groundwork for the formal verification of DEVS models based on their transformation into TA. Based on this basic ability to verify DEVS models, further enhancements to the methodology are possible. Such enhancements would increase the model checking efficiency by decreasing the total state-space traversed during the model checking analysis. Such increase in efficiency would translate into a practical ability to verify larger DEVS models than what is currently possible, thus making formal DEVS verification possible for industrial-size models.

These enhancements are based on exploiting some properties that are specific to the TA models resulting from the transformation of DEVS models. Such properties are not valid in general in TA. One of these properties is the nature of any embedded DEVS execution engine such as $\mathrm{e}-\mathrm{CD}++$. These engines usually run on a single processor machine and thus use a single physical clock to measure the elapsed time for all atomic DEVS components executing on this platform. Another observed property of many practical DEVS models is the re-use of the same atomic or coupled component many times in a complex DEVS model. Thus, the use of symmetry reduction techniques would reduce the number of distinct components to be verified. We expand on these two main possible future directions below:

- Reducing state-space through reducing model complexity. Model checking timed automata models depends on building a finite reachability graph called Region Graph. Answering queries about system properties is done through 
traversing this graph and validating the desired property at each node. Thus the complexity of the reachability algorithm depends on the size of the region graph. Aceto and Laroussinie [83] showed that this size is in the order of $\mathrm{O}\left(|\mathrm{C}| !+\mathrm{M}^{|C|}\right)$, where $|\mathrm{C}|$ is the total number of clocks in both the TA model and the query, and $\mathrm{M}$ is the maximum constant appearing in the TA model or the query. Hence a great reduction in region graph size can be achieved by reducing the number of clocks in a TA model. This may be done in several ways as follows:

- Checking safety and reachability properties of a DEVS model on an abstraction of untimed finite automata. This untimed finite automata model would be obtained by removing all the timing information from the DEVS model. This untimed version would constitute a rough abstraction of the DEVS model that contains all behaviors of the original DEVS model, plus some extra behavior due to removing the restrictions of timings. However, checking untimed model would be much more efficient than the timed model. This approach does not work for checking bounded-liveness properties on DEVS models. If verifying these properties is required, then next optimization would help.

- In our methodology, as well as in all others as introduced in literature, each atomic DEVS model is transformed to a TA model with a clock 
that measures the model elapsed time. One-clock automata can use efficient model-checking algorithms, as shown in [84]. However, any coupled DEVS model would be composed of many atomic models, each with its own clock. Therefore, the resulting equivalent composed TA for the coupled model would contain more than one clock, and thus cannot be considered a one-clock TA model. On the other hand, in most embedded simulators and runtimes, the coupled DEVS model would execute on a single processor using a single global clock. This assumption can be used to obtain an equivalent TA model with only one global clock for the complete coupled model. This would enable the efficient verification of such TA models, and thus scale up the size and number of DEVS components that can practically been verified. If the assumption of single processor cannot be guaranteed, as in the case in clustered systems for example, we would need a single clock for each component, and this optimization technique would not apply.

- Symmetry in DEVS coupled models could be used to reduce state-space. As any typical system is normally composed of many components, it is common to find the same type of component replicated in many places in the system. A DEVS model of such system would contain replicated components. An intelligent conversion method from DEVS to TA could identify identical 
DEVS components, and flag them for symmetrical analysis in the resulting TA model, thus greatly reducing state-space during reachability analysis.

Another possible future research is related to the verification of continuous systems modeled with the QSS method. As described in section 5.1.3, the QSS method approximates the actual system trajectory. This approximation results in an error $|E|$ which is the difference between the true system trajectory and the QSS approximated trajectory as can be seen in Figure 46 . We can find a bound on this error as per [51] that depends on the quantization step $d Q$. For formal verification of hybrid system, it is desirable to verify the true system trajectory against safety requirements, and not only the approximation. To extend our verification methodology to do this, we can increase QSS intervals outwards with the estimated approximation error $|E|$. This would guarantee the true system trajectory is covered inside the trajectory being verified. Future research would look into formal models for this enhancement, effect of this on the verification complexity and the TA implementation. In case of highly non-linear functions, the error $|E|$ can be large. In this case, another extension could be using QSS2 method in which the system trajectory is approximated with second order segments as shown in [51]. 


\section{REFERENCES}

[1] M. J. Rehman, F. Jabeen, A. Bertolino, and A. Polini. 2007. "Testing Software Components for Integration: a Survey of Issues and Techniques". Software Testing, Verification and Reliability 17(2): 95-133.

[2] R. Gerlich, R. Gerlich, T. Boll. 2007. "Random Testing: From the Classical Approach to a Global View and Full Test Automation". In Proceedings of the 2nd international Workshop on Random Testing, Co-Located with the 22nd IEEE/ACM international Conference on Automated Software Engineering (ASE 2007), Atlanta, Georgia.

[3] M. B. Dwyer, J. Hatcliff, R. Robby, C. S. Pasareanu, and W. Visser. 2007. "Formal Software Analysis Emerging Trends in Software Model Checking". In Proceedings of the 2007 Future of Software Engineering (FOSE '07). IEEE Computer Society, Washington, DC, pages 120-136.

[4] S. Kowalewski. 2002. "Introduction to the Analysis and Verification of Hybrid Systems". Modelling, Analysis, and Design of Hybrid Systems. Lecture Notes in Control and Information Sciences, 279: 153-171.

[5] R. Alur, D. Dill. 1994. "Theory of Timed Automata". Theoretical Computer Science, 126: 183235.

[6] G. Wainer, E. Glinsky, and P. MacS Ien. 2005. "A Model-Driven Technique for Development of Embedded Systems Based on the DEVS Formalism". In Model-driven Software Development Volume II of Research and Practice in Software Engineering, edited by S. Beydeda and V. Gruhn. Springer-Verlag.

[7] B. P. Zeigler, T. Kim, and H. Praehofer. 2000 . Theory of Modeling and Simulation. San Diego, CA: Academic Press, ISBN-10: 0127784551. 
[8] Y. H. Yu, G. Wainer. 2007. "eCD++: an engine for executing DEVS models in embedded platforms". In Proceedings of the 2007 SCS Summer Computer Simulation Conference, San Diego, CA July 15-18.

[9] G. Behrmann, A. David, K Larsen. 2004. "A Tutorial on Uppaal". Proceedings of the 4th International School on Formal Methods for the Design of Computer, Communication, and Software Systems. LNCS 3185.

[10] J. Bengtsson, W. Yi. 2004. "Timed Automata: Semantics, Algorithms and Tools". Lectures on Concurrency and Petri Nets, 3098.

[11] G. Wainer. 2002. "CD++: a toolkit to develop DEVS models". Software: Practice and Experience 32 (13): 1261-1306.

[12] E. Kofman, M. Lapadula, and E. Pagliero. 2003. "Powerdevs: A devs-based environment for hybrid system modeling and simulation". Technical Report LSD0306, School of Electronic Engineering, Universidad Nacional de Rosario, Rosario, Argentina.

[13] Muzy, J. Nutaro. 2005. "Algorithms for efficientcient implementations of the devs \& dsdevs abstract simulators". In proceedings of 1st Open International Conference on Modeling \& Simulation: 401-407, ISIMA / Blaise Pascal University, France, June 2005.

[14] B. P. Zeigler, H. Sarjoughian. 2002. "Introduction to DEVS modeling and simulation with JAVA: A simplified approach to HLA compliant distributed simulations". Tucson: University of Arizona, Accessed March 2011. http://www.acims.arizona.edu

[15] X. Hu; B.P. Zeigler, J. Couretas. 2001. "DEVS-on-a-chip: implementing DEVS in real-time Java on a tiny Internet interface for scalable factory automation". In: proceedings of IEEE 
International Conference on Systems, Man, and Cybernetics, Tucson, AZ, USA, 2001(5): 3051 3056.

[16] F. Bergero, E. Kofman. 2011. :PowerDEVS: a tool for hybrid system modeling and real-time simulation". SIMULATION 87 (1-2): 113-132.

[17] Cho, Y. K., X. Hu, and B. P. Zeigler. 2003. The RTDEVS/CORBA environment for simulationbased design of distributed real-time systems. SIMULATION 79 (4).

[18] F. R. Sadeghi, G. Wainer, and M. Moallemi. 2010. "Modeling and Controlling a Robotic Arm with E-CD++". Poster, SummerSim 2010 Conference - July 2010.

[19] A.Valmari. 2009. "Software model checking is a rich research field". International Journal on Software Tools for Technology Transfer (STTT) 11(1): 1-11.

[20] L. Aceto, A. Bergueno, and K. G. Larsen. 1998. "Model Checking via Reachability Testing for Timed Automata". In Proceedings, Fourth Workshop on Tools and Algorithms for the Construction and Analysis of Systems, Lisbon, Portugal, LNCS 1384.

[21] R. Alur, C. Courcoubetis, D. L. Dill. 1990. "Model-Checking for Real-Time Systems". In Proceedings, Fifth Annual IEEE Symposium on Logic in Computer Science, Philadelphia, PA.

[22] R. Alur, C. Courcoubetis, D. L. Dill. 1993. "Model-Checking in Dense Real-Time". J. of Information and Computation 104(1): 2-34.

[23] T. A. Henzinger, X. Nicollin, J. Sifakis, S. Yovine. 1992. "Symbolic Model Checking for RealTime Systems". Proc. of 7th Annual IEEE Symposium on Logic in Computer Science, Santa Cruz, CA. 
[24] T. A. Henzinger, X. Nicollin, J. Sifakis, S. Yovine. 1994. "Symbolic Model Checking for RealTime Systems". Journal of Information and Computation 111(2): 193-244.

[25] P. Bouyer and F. Laroussinie. 2008. "Model Checking Timed Automata". In Modeling and Verification of Real-Time Systems, edited by N.Navet and S. Merz 111-140. ISTE Ltd. - John Wiley \& Sons, Ltd.

[26] G. Wainer, L. Morihama, and V. Passuello. 2002. "Automatic verification of DEVS models", In Proceedings of SISO Spring Interoperability Workshop, Orlando, FL. U.S.A. March 10-15.

[27] Hernandez, N. Giambiasi. 2005. "State Reachability for DEVS Models", Proc. of Argentine Symposium on Software Engineering (2005).

[28] J.S. Hong, H.S: Song, T.G. Kim, K.H. Park. 1997. "A realtime Discrete Event System Specification formalism for seamless real-time software development". Discrete Event Dynamic Systems 7 (4): $355-75$.

[29] H.S. Song, T.G. Kim. 2005. "Application of Real-Time DEVS to Analysis of safety-Critical Embedded Control Systems: Railroad Crossing Control Example", SIMULATION 81(2):119-136.

[30] SP-DEVS on Wikipedia accessed Feb.28, 2011 at http://en.wikipedia.org/wiki/SP-DEVS.

[31] M. H. Hwang. 2005. "Tutorial: Verification of Real-time System Based on Schedule-Preserved DEVS". In Proceedings of 2005 DEVS Symposium, San Diego, April 2-8.

[32] M. H. Hwang. 2005. "Generating Finite-State Global Behavior of Reconfigurable Automation Systems: DEVS Approach". In Proceedings of 2005 IEEE-CASE, Edmonton, Canada, Aug. 1-2.

[33] M. H. Hwang, S.K. Cho, B.P. Zeigler, and F. Lin. 2007. "Processing Time Bounds of SchedulePreserving DEVS". Arizona Center of Integrative Modeling \& Simulation Technical Report. 
[34] M-H. Hwang, B.P. Zeigler. 2009. "Reachability Graph of Finite and Deterministic DEVS Networks". IEEE Transactions on Automation Science and Engineering 6 (3): 468 - 478.

[35] A. Furfaro, L. Nigro. 2008. "Embedded Control Systems Design based on RT-DEVS and temporal analysis using UPPAAL". Proceedings of Computer Science and Information Technology, 20-22 Oct. 2008, IMCSIT 2008: 601-608.

[36] A. Furfaro, L. Nigro. 2009."A development methodology for embedded systems based on RTDEVS". Innovations in Systems and Software Engineering 5: 117-127.

[37] F. Cicirelli, A. Furfaro, L. Nigro, and F. Pupo. 2010. "Temporal verification of RT-DEVS models with implementation aspects". In Proceedings of the 2010 Spring Simulation Multiconference (SpringSim'10), Orlando, FL, USA, April 11 - 15, 2010.

[38] S. Han, K. Huang. 2007. "Equivalent Semantic Translation from Parallel DEVS Models to Time Automata", In Proceedings of the 7th international conference on Computational Science, Part I. ICCS 2007 (ICCS '07).

[39] L. Aceto, A. Ingólfsdóttir, K. Guldstrand Larsen, J. Srba. 2007. Reactive Systems: Modelling, Specification and Verification. Cambridge University Press.

[40] H. Dacharry, N. Giambiasi. 2007. "Formal Verification Approaches for DEVS". Proceedings of Summer Computer Simulation Conference, San Diego, CA, USA July 15-18, 2007.

[41] N. Giambiasi, J-L. Paillet, F. Châne. 2003. "From Timed Automata To DEVS Models". Proceedings of the 2003 Winter Simulation Conference, Phoenix, AZ, Dec. 7-10.

[42] K.J. Hong T. G. Kim. 2005. "Timed I/O Test Sequences for Discrete Event Model Verification". Artificial Intelligence and Simulation 3397: 275-284. 
[43] Y. Labiche, G. Wainer. 2005. "Towards the Verification and Validation of DEVS Models". Proceedings of the 1st Open International Conference on Modeling \& Simulation. ClermontFerrand, France: $295-305$.

[44] R. Castro, E. Kofman, and G. A. Wainer. 2008. "A formal framework for stochastic DEVS modeling and simulation". In Proceedings of the 2008 Spring simulation multiconference (SpringSim '08). Society for Computer Simulation International, San Diego, CA, USA, 421-428.

[45] M. Kwiatkowska, G. Norman, R. Segala, and J. Sproston. 2002. "Automatic verification of realtime systems with discrete probability distributions". Theor. Comput. Sci. 282, 1 (June 2002), 101 150.

[46] M. S Branicky. 2005. "Introduction to Hybrid Systems" D. Hristu-Varsakelis and W.S. Levine (eds.), Handbook of Networked and Embedded Control Systems, 91-116. Boston: Birkhauser.

[47] A. Donzé, O. Maler. 2007. "Systematic simulation using sensitivity analysis". In Proceedings of the 10th international conference on Hybrid systems: computation and control (HSCC'07):174189.

[48] A. Donzé. 2007. "Trajectory-Based Verification and Controller Synthesis for Continuous and Hybrid Systems". PhD thesis, University Joseph Fourier.

[49] A. Donzé, B. Krogh, and A. Rajhans. 2009. "Parameter synthesis for hybrid systems with an application to simulink models". In Proceedings of the 12th International Conference on Hybrid Systems : Computation and Control (HSCC'09), San Francisco, CA, USA, April 13-15, 2009.

[50] E. Kofman, S. Junco. 2001."Quantized State Systems. A DEVS Approach for Continuous Systems Simulation". Transactions of SCS. 18(3): 123-132. 
[51] E. Kofman. 2004. "Discrete Event Simulation of Hybrid Systems". SIAM Journal on Scientific Computing 25(5): 1771-1797.

[52] M. Otter, F. Cellier. 1996. The Control Handbook, chapter Software for Modeling and Simulating Control Systems, 415-428. CRC Press, Boca Raton, FL.

[53] G. Decknatel, R. Slovák, E. Schnieder. 2002. "Definition of a Type of Continuous-Discrete HighLevel Petri Nets and Its Application to the Performance Analysis of Train Protection Systems In S. Engell, G. Frehse, and E. Schnieder (Eds.), Modelling, Analysis, and Design of Hybrid Systems, Lecture Notes in Control and Information Sciences 279: 355-367.

[54] T. Henzinger. 1996. "The Theory of Hybrid Automata". Lecture Notes in Computer Science 278.

[55] T. A. Henzinger, P. W. Kopke, A. Puri, and P. Varaiya. 1995. "What's decidable about hybrid automata?". In Proceedings of the twenty-seventh annual ACM symposium on Theory of computing, STOC '95, New York, NY, USA, 373-382.

[56] J. Lunze and J. Raisch. 2002. "Discrete Models for Hybrid Systems. Modelling, Analysis, and Design of Hybrid Systems", Lecture Notes in Control and Information Sciences, 279: 67-80.

[57] R. Alur, T.A. Henzinger, G. Lafferriere, G.J. Pappas. 2000. "Discrete abstractions of hybrid systems". Proceedings of the IEEE, 88(7): 971-984.

[58] E. Barke, D. Grabowski, H. Graeb, L. Hedrich, S. Heinen, R. Popp, S. Steinhorst, and Y. Wang. 2009. "Formal approaches to analog circuit verification". In Proceedings of the Conference on Design, Automation and Test in Europe (DATE '09), European Design and Automation Association, 3001 Leuven, Belgium, Belgium, 724-729. 
[59] Oded Maler and Grégory Batt. 2008. "Approximating Continuous Systems by Timed Automata". In Proceedings of the 1st international workshop on Formal Methods in Systems Biology (FMSB '08), Cambridge, UK, Jasmin Fisher (Ed.). Springer-Verlag, Berlin, Heidelberg, 77-89.

[60] F. Cellier, E. Kofman. 2006. Continuous System Simulation. ISBN 978-0-387-26102-7, Springer publishing.

[61] J. G. Rokne. 2001." Interval arithmetic and interval analysis: an introduction". In Granular computing, Witold Pedrycz (Ed.). Physica-Verlag GmbH, Heidelberg, Germany.

[62] B. Hayes, 2003."Lucid Interval", Scientific American, November-December 2003, 91(6): 484.

[63] R. E. Moore, R. B. Kearfott, and M. J. Cloud, 2009."Introduction to Interval Analysis". Society for Industrial and Applied Math., Philadelphia, PA, USA.

[64] J. Srba. 2008. "Comparing the Expressiveness of Timed Automata and Timed Extensions of Petri Nets". In Proceedings of the 6th international conference on Formal Modeling and Analysis of Timed Systems (FORMATS '08), Franck Cassez and Claude Jard (Eds.). Springer-Verlag, Berlin, Heidelberg, pages 15-32.

[65] V. Gupta, T. A. Henzinger, R. Jagadeesan. 1997. "Robust timed automata". Hybrid and Real-Time Systems 1201: 331-345.

[66] M. De Wulf, L. Doyen, N. Markey. 2004."Robustness and Implementability of Timed Automata" Formal Techniques, Modelling and Analysis of Timed and Fault-Tolerant Systems : 359-374.

[67] M. De Wulf, L. Doyen, J-F. Raskin. 2004. "Almost ASAP Semantics: From Timed Models to Timed Implementations" Hybrid Systems: Computation and Control : 296-310. 
[68] H. Saadawi, G. Wainer. 2010. "Rational time-advance DEVS (RTA-DEVS). In Proceedings of DEVS Symposium 2010, Orlando, FL., April 11-15.

[69] J. Miller. 2000. "Decidability and complexity results for timed automata and semi-linear hybrid automata". Hybrid Systems: Computation and Control, LNCS 1790.

[70] H. Saadawi, G. Wainer. 2011. "Principles of DEVS Models Verification". SIMULATION: Transactions of the Society for Modeling and Simulation International, October 23, 2011, doi: $10.1177 / 0037549711424424$.

[71] H. Saadawi, G. Wainer, M. Moallemi. 2012. "Principles of DEVS Models Verification for RealTime Embedded Applications". Real-Time Simulation Technologies: Principles, Methodologies, and Applications. K. Popovici, P. Mosterman Eds. Taylor and Francis. CRC Press. 2012.

[72] H. Saadawi, G. Wainer. 2009. "Verification of real-time DEVS models". In Proceedings of DEVS Symposium 2009. San Diego, CA, March $22-27$.

[73] H. Saadawi, G. Wainer. 2010. "From DEVS to RTA-DEVS". In Proceedings of the 2010 IEEE/ACM 14th International Symposium on Distributed Simulation and Real Time Applications (DS-RT'10), Fairfax, VA. USA, Oct.17-20: 207-210.

[74] C. Daws, A. Olivero, S. Tripakis, and S. Yovine. 1996. "The tool KRONOS". In Proceedings of the DIMACS/SYCON workshop on Hybrid systems III : verification and control: verification and control, Secaucus, NJ, USA, 208-219.

[75] S.Yovine. 1997. "Kronos: A verification tool for real-time systems". Springer International Journal of Software Tools for Technology Transfer 1(1-2): 123-133.

[76] Wikipedia. DEVS behavior. http://en.wikipedia.org/wiki/ Behavior_of_Coupled_DEVS [Accessed: Aug. 2009]. 
[77] H. Bowman, R. Gomez. 2006. Concurrency Theory: Calculi and Automata for Modelling Untimed and Timed Concurrent Systems. Springer-Verlag London.

[78] Puri. 1998. "Dynamical properties of timed automata", Formal Techniques in Real-Time and Fault-Tolerant Systems 210-227.

[79] http://en.wikipedia.org/wiki/Exponential_decay wikipedia, 2011, "Exponential decay", accessed Sept. $2^{\text {nd }} 2011$.

[80] B. Berard, , M. Bidoit, A. Finkel, F. Laroussinie, A. Petit, L. Petrucci, Ph. Schnoebelen and P. McKenzie. 2001. Systems and Software Verification: Model-Checking Techniques and Tools. Springer Verlag.

[81] H. Saadawi, G. Wainer. 2012. "On the verification of hybrid DEVS models". In Proceedings of the 2012 Symposium on Theory of Modeling and Simulation - DEVS Integrative M\&S Symposium (TMS/DEVS '12), Orlando, FL,USA. March 26-28.

[82] Henzinger, T.A.; Pei-Hsin Ho; Wong-Toi, H.; , "Algorithmic analysis of nonlinear hybrid systems," Automatic Control, IEEE Transactions on, vol.43, no.4, pp.540-554, Apr 1998.

[83] L. Aceto and F. Laroussinie, 2002. "Is your model checker on time? On the complexity of model checking for timed modal logics". Journal of Logic and Algebraic Programming, 52-53:7-51, August 2002.

[84] F. Laroussinie, N. Markey and Ph. Schnoebelen. "Model Checking Timed Automata with One or Two Clocks". CONCUR 2004 - Concurrency Theory, Lecture Notes in Computer Science 3170 : $387-401$. 